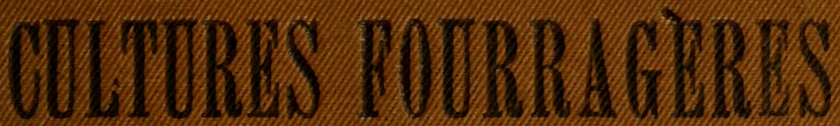

\section{P ATUIRAGLS}

os

\section{PMLOUS}

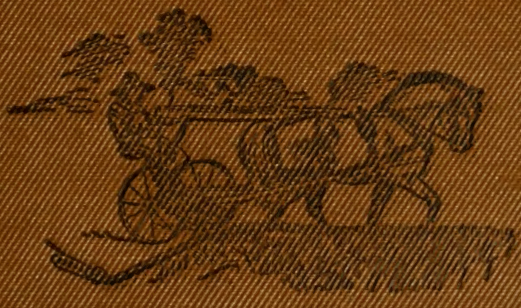

- 10

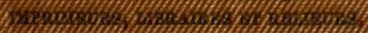

1) 

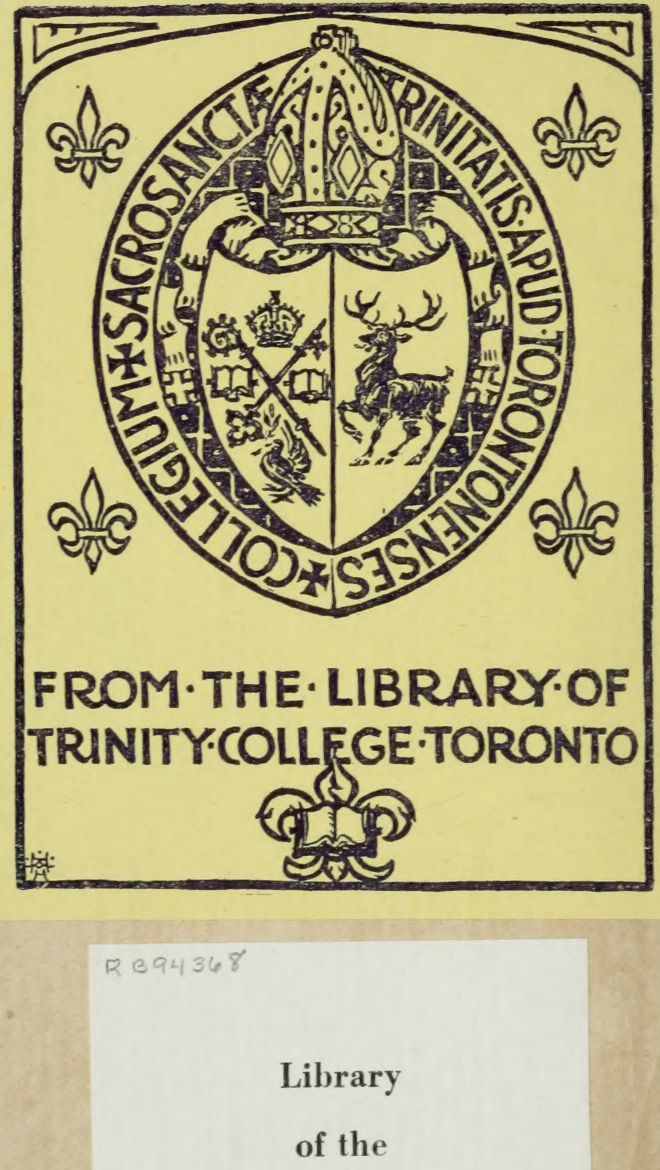

University of Toronto 



\section{CULTURES FOLRRACÈRES}

\section{PATURAGES}

ET

\section{PELOUSES}

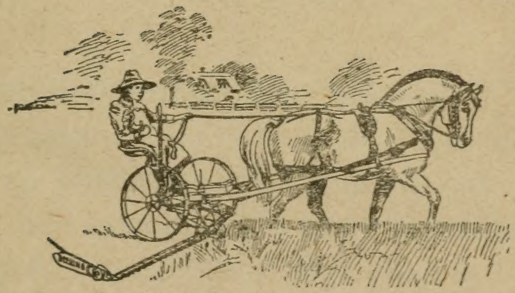

MERCIER \&\& CIE IEPRIMEURS, LIBRAIRES, RELIEURS, ETC.

17-23. Côte du Passage İ́vis 
Enregistré conformément à l'acte du Parlement du Canada ... l'année mil huit cent quatre-vingt seize, par J. B. Plante, au bureau du ministre de l'Agriculture à Ottawa. 


\section{INTRODUCTION}

Dans la province de Québec, l'agriculture est en train de subir une transformation, nécessitée par les changements profonds qui, dans ces derniers temps, se sont produits dans l'économic rurale de certains pays, ainsi que dans les conditions du trafic international. Depuis l'ouverture des immenses contrées à blé: Manitoba, Territoires du Nord-Ouest, Etats-Unis, les Indes, les prix du blé sont déterminés par des facteurs qui ont leur siège dans des régions le plus souvent bien éloignées de chez nous. La concurrence que nous en éprouvons fait que ces prix sont plus bas qu'ils ne l'étaient il y a une vingtaine d'années pendant que nous payons plus cher la terre et la main-d'œuvre et que les frais généraux d'exploitation vont toujours en augmentant. Mais le revenu de la culture des céréales se réduisant ainsi de plus en plus, il s'ensuit naturellement que l'agriculteur s'adonne darantage à celle des fourrages. $\Lambda$ ussi cette branche de l'éeonomie rurale prend-elle, dans notre province, un déve 
loppement qui va grandissant d'année en année et bientôt elle l'emportera sur toutes les autres. Cependant, malgré la haute importance qu'on lui reconnait, ros cultivateurs ont encore beaucoup à apprendre pour pratiquer rationnellement cette exploitation agricole.

Dans le présent travail, nous allons nous efforcer d'expliquer, en style simple et intelligible à tous, de la manière la plus détaillée et la plus compiète, comment il importe de procéder pour constituer des prairies d'un rendement considérable. Les préceptes énoncés sont fondés sur une grande quantité d'observations scientifiques recueillies par les stations expérimentales et de contrôle des semences, dans différents pays ainsi que sur les expériences agricoles faites avec divers mélanges de graines fourragères dans les conditions les plus diverses.

Nous avons cru devoir entrer dans bien des détails sur les méthodes suivies dans d'autres pays qui ne sembleront peut-être pas praticables dans le nôtre, mais notre but étant de faire une étude aussi complète que possible de la question, ces digressions étaient nécessaires.

Tout cultivateur soucieux de ses intérêts fera donc bien d'étudier la composition des mélanges 
dont il est question dans cet ouvrage, en ayant soin de ne pas les prendre comme des recettes invariables, mais comme des exemples propres à l'éclairer sur la matière et à lui apprendre à composer lui-même les mélanges les mieux appropriés aux conditions locales dans lesquelles il travaille.

L'ouvrage comprend les divisions suivantes:

I. Réduction de la culture des céréales et extension de la culture fourragère.-Production du blé dans les prairies de l'Ouest.

II. Conditions climatériques de la culture fourragère.

III. Engazonnement naturel.

IV. Semis de graine de foin.

V. Théorie et calcul des mélanges.

VI. Choix des plantes.

VII. Mélanges de graines fourragères.

1. Trèfles et graminées.

2. Mélanges pour prairies temporaires.

3. Mélanges pour prairies permanentes

4. Considérations générales.

VIII. Achat des semences.

IX. Préparation du terrain.

1. Du sol.

2. Du sous-sol.

3. De la configuration du terrain.

4. Du drainage.-Assainissement. 
X. Culture préparatoire ou récoltes précédentes, à base de plantes annuelles, sarclúes et améliorantes.-Engrais et préparation du sol.

1. Engrais.

2. Engrais liquides.

3. Comment engraisser le sol.

et les limons.

4. Les composts, les balayures, les boues

5. Restitution du sol.

6. Saison propice à la restitution du sol.

XI. Epoque des semailles. - Diverses manières de semer.-Semailles sans grain.

XII. Semailles avec grain.-Récoltes protectrices.-Enterrement de la semence.

A. En terre torte.

B. En terre légère.

XIII. Soins d'entretien des prairies.

\section{Destruction des mousses}

Nous avons en outre ajouté quelques chapitres touchant l'utilité des prairies et les meilleurs moyens à employer pour en augmenter le rendement, tant sous le rapport de la quantité que sous celui de la qualité. Nous avons anssi étudié l'herbe sous le rapport de sa valeur pour la nourriture du bétail.

Ces chapitres ont pour titres: 
XIV. L'herbe.

1. Importance d'un bon pâturage.

2. Qualités nutritives de l'herbe des prés.

อิ. Foin.

4. L'herbe et le foin, fourrages à encou-

rager.

XV. Exploitation et produit des prairies.

1. Alternance des modes d'exploitation.

2. Fauchage des prairies tous les ans.

3. Prairies et pâturages.

4. Alteinat des pâturages:

XVI. Fauchage des prairies.-La fenaison.

XVII. Les fourrages verts.

De plus, nous arous joint au présent trarail, une étude qui se rapproche sous tous les rapports du sujet déjà traité, nous roulons parler des "Gazons d'agrément."

Ce sujet comporte les points suivants :

XVIII. Les belles pelouses des parcs et des jardins.

1. Préparation du terrain, tasseme nt.

2. Semis.

3. Le sarclage.

4 La tonte. 


\section{RÉDUCTION}

DE LA

\section{CULTURE DES CÉRÉ ALES}

ENTENSTON DE LA CULTIRE FOUPRAGERE

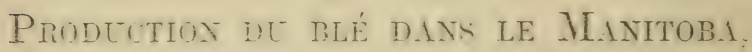
les terititones du NohD-Ouest ET LES ÉTATS-UNIS.

Autrefois, la prorince de Québec récoltait le blé nécessaire à sa consomliation. Il n'en est plus de mème aujourd'hui. En effet, les immenses territoires de l'ounst produisent des millions de minots de blé à un pris de revient nominal.

Sur tous cus domainas, il n'y a que les bâtiments les plus indispensables pour le logement des ourriers et le remisaģe des machines et outils quelconques. Il n'existe que peu de petites fermes. Sur les grands domaines, la plupart des 
travaux sont exécutés par des machines, et, par conséquent, ue coûtent pas cher.

Au petit fermier, il manque souvent le capital nécessaire pour se procurer l'outillage nécessaire, ce qui l'entrave et le rend incapable de se soutenir longtemps.

Comme dans les grands domaines on ne possède généralement presque pas de raches, de bœuís ou de chevaux et que les bêtes de trait se bornent à des mulets très mal soignés, il n'y reste en hiver que peu de personnes, sourent dix hommes seulement sur des propriétées d'une très grande étendue. A l'époque des semailles, durant tout le mois d'avril, on engage exprès de cent à deux cents ouvriers et, de noureau, de deux cents à trois cents pour la moisson, du ler août au 15 septembre. Dans l'eutre-temps, ces paurres gens restent à peu près inoccupés. - Il n'est pas question de restituer au sol les éléments nutritifs enlerés par les récoltes, le fertile terrain des prairies pouvant donner les plus riches rendements de froment pendant une longue suite d'années, sans receroir de fumure d'aucune sorte. Il est évident que, dans de telles circonstances d'exploitation, les frais de production sont réduits à peu de chose. Le prix de la terre est à peu près nul, de sorte que 
les frais ne consistent principalement que daus les intérêts et l'a mortissement du capital des machines. Et depuis ces derniers temps, le commerce fournit ces machines, à la fois puissantes et pratiques, à un prix relativement très bas.

Pour ces causes, les frais de production se réduisent en un sibas pris que nous ne pourons en aucune manière soutenir la concurrence. Il est de fait que la culture du blé ne paie plus dans notre prorince. Aussi, y a-t-il tendance générale à laisser la culture du froment pour s'adomner à celle des plantes fourragères

Nous ne pourons lutter contre les prairies de l'ouest pour la production du blé mais nous u'arons pas de riraux possibles pour la production des fourrages.

II. Conditions slimatrinutes de la culture fourragère.

C'est le régime des pluies qui exerce la plus grande influence sur la régétation herbacée d'une contrée. Plus elles sont fréquentes et aboudantes, plus aussi est intense la pousse des graminées saurages et cultirées, à condition d'ailleurs que la température soit favorable.

Notre prorince est bien partagée sous ce rapport. L'immense vallée du Saint Laurent est, (m) Anérique, la zóne par excellence pour les four. 
rages et les pâturages; à nous d'en profiter. L'industrie laitière n'est pas seulement obligatoire pour nous; elle est, de plus, d'une graude facilité.

\section{Engazonnement naturel.}

Il parait qu'en certains pays et même en certains cas dans notre province on se borne après la moisson des céréales d'abandonner à elle-même la terre destinée à la culture du fourrage.

Le sol s'engazonne plus ou moins dans la première année; mais celle produclion ne consiste qu'en graminées médiocres, entremélées de manvaises herbes très nombrenses. Si l'on examine un "pré naturel" de cette sorte, on roit que les graminées dominantes sont le palurin commun (Poa trivialis, L.) ainsi que la houlque laincuse (Holcus louatus, L.) qui est une espèce de très peu de valeur. Si l'on s'imag̣ine en aroir récolté une grande quantité, l'on est dans l'erreur car le recours à la balance réduit très fort ces belles illusions. A cause des poils dont les feuilles sont revêtues, la houlque laineuse n'est pas mangée volontiers par le bétail, quoique le contraire soit affirmé par certains écrivains agricoles. A côté de cette espèce, l'on roit la flonve odorante (Anthoxanthum odoratum, L.) qui ne vaut guère mieux et dont la semence mûrit dans la céréale et tombe: 
elle aussi ne donne qu'un fourrage médiocre. Le sol de ce pré pitoyable est recourert par les tiges, étendues en tous sens, de la renoncule rampante (Renunculus repens, L.) à laquelle est associée d'ordinaire la petite oseille (Rumex autosella, L.), et l'une et l'autre sont des herbes très nuisibles. Ça et là se troure aussi la forme du fromemtal. (Areua elatior, L.) dite avoine is chapelet, distinguée par les renflements superposés du pied de sa tige, et dont les racines ont persisté dans le champ, même après plusienrs années de culture. Voilà l'état du pré, la première année. Mais celui-ci change immédiatement et d'uue manière arantageuse après qu'on l'a gratifié d'un premier arrosement de purin ainsi que la chose se pratique dans la plupart des pays d'Europe. Cet excellent engrais liquide contient une quantité de graines, nolamment de trèfle rouge et blanc encore capable de germer, qui, sorties de l'organisme animal sans aroir été dig'érées, sont arrirées dans le purin arec les réjections solides.

Pour donner une idée de l'importance de l'arrosage d'une prairie aree du purin, comme semis, on a fait l'expérience suivante:

On garda et l'on mit sécher le dépôt resté dans une tomne à purin après un arrosage. L'examen qui en fut fait constata que ce résidu consistait en 98 o/o de terre, mais arec laquelle il 
y avait aussi 1,19 o/0 de graines diverses. Un fourrage, on le sait, contient toujours des semences mûres, et c'est notamment le regain qui est très riche en graines parfaitement développées, surtout des trèfles rouge et blanc. Celles-ci passent arec le fourrag'e dans l'estomac de l'animal mais, comme elles ne sont digérées qu'en partie et qu'uue certaine quantité ressort de l'intestin, arec toute sa ritalité, on s'explique comment ces graines se trouvent dans le purin.

Une livre du résidu en question contenait :

Trefle blane.....5.908 grains

Trèfle rouge..... 721 "

Lupuline........ $13 \quad$ "

Gesse des prés....

Petite oseille.....

Plantain lancéolé.

Oseille des prés...

Quintefeuilie......

Myosotis, ........

Paturin............

Ray-grass anglais.

Renoué persicairr

Brunelle...........

Sétaire glauque...

Centaurée des prés

$\begin{array}{rr}2 & . \\ 94 & \text { " } \\ 47 & \text { " } \\ 18 & \text { " } \\ 42 & \text { " } \\ 26 & \text { " } \\ 32 & \text { " } \\ 21 & \text {. } \\ 10 & \text {. } \\ 11 & \text { " } \\ 10 & \text { " } \\ 8 & \text { " }\end{array}$

Ifoulqu laineuse. . \& graines

Alysson.......... 5 "

Pissenlit......... 5

Floure odorante... 5

Renoncule âcre.... 5

Cumin des prés.... 2

Ansérine........ 2

Fromental......... 3

Timothy........ 3

Carotte sauvage.... 3

Esprion le silin.....?

Galéonir des champes.?

Persil d'âne........ ? "

Graines de raisin.. 2

3 inconnues......29

Total 32 espèces en 7,038 graines.

Si l'on compte que chaque tonne de purin, de la contenance de 100 gallons, ne contient qu'une livre de ce résidu, mais qu'en deux fois l'an, dans les pays où l'excellente méthode d'épandage du 
purin est pratiquée, un acre de prairie reçoit la décharge de trente-trois de ces barils, il suit que de cette manière il n'arrire sur la prairie pas moins de 232.254 graines, qui proriennent presque toutes des trèfles blanc et rouge, puisque celles-ci sont au nombre de 218,757 .

Les graines de ces trèfles recueillies du résidu du purin ont été examinées arec le plus grand soiu pour déterminer leur faculté germinative. En dix-huit jours.

Trèfle blanc

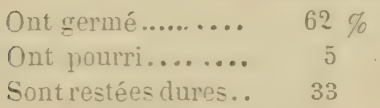

Total...... $\overline{100} \%$
Trèlle rouge

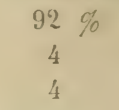

$\overline{100} \%$

Si, de ces graines restées dures, l'on admet comme capables de germer, un tiers de celles du trèfle blane et la moitié de celles du trèfle rouge, et quon les porte aussi en compte, il résulte que la faculté gorminative est pour la première de it olo. Celle-ei y atteint done un chiffre qui ne sobserve que whez les meilleures qualités de la senence du commerce. Cette faculté germinative ne se serait pas trourée aussi grande chez les autres graines, parce qu'elles araient ell partie souffert beaucoup ou araient été déformées au point d'étre méconnaissables. 
Il est vrai que celle des trèfles n'auraient pas présenté un degré si haut de faculté germinative, si elles avaient été essayées immédiatement après avoir été retirées de la tonne à purin; mais c'est là une question qui a besoin de recherches ultérieures. Quand elles passent de la tonne surla prairie, où elles sont exposées à des alternatives ultérieures d'humidité et de sécheresse, de chaleur et de froidure, la faculté g’erminative n'en est cer. certainement pas moins grande.

Cequi vient d'être exposé prouve de nouveau la haute valeur des engrais liquides pour les prairies; nous royons par là qu'ils ne s'en tiemnent pas seulement à les doter de principes que la chimie démontre être indispensables à la nutrition des plantes, mais que leur influence farorable consiste aussi en grande partie dans l'apport de nombreux organismes végétaux. Du reste, personue n'est mieux convaincu de ces rérités que le paysan de la Suisse, qui estime que pour un nouvel engiazonnement de son pré, un arrosement au purin vaut autant que de l'ensemencer à moitié de trèfles. Il résulte des déterminations botaniques communiquées ci-dessus, que la collection de graines contenue dans le purin est très uniforme et se compose comme on l'a constaté pratiquement, en majeure partie de trèfles blanc et rouge, surtout du premier. 
Les cultirateur's feraient done mieux de commencer par semer un mélange convenable de graines afin d'obtenir un bon rapport dès les premières années. Par là, la semence de purin ne sera nullement perdie: car, comme on le sait, le reudement des trèfles étant sujet à diminuer aprés la deuxième amnée déjà,il est utile d'en repourroir constamment Ia prairie par les apports de cet engrais liquide.

Si les excréments solides sont portés au fumier, où ils restent pendant plusieurs mois, les graines y périssent en partie. En outre celles qui résistent perdent toute leur valeur, parce que, le plus sourent, le fumier est enfoui au labour et si profondément que les graines fines sont mises dans l'impossibilité de germer. Et même si elles lèrent, elles produisent des plantes qui doirent être regardées comme des mauraises herbes préjudiciables à la culture du pré.

La conséquence de l'emploi du purin est que, dès le premier arrosag'e, la végétation de la jeune "prairie naturelle" prend un aspect plus satisfaisant et qu'elle gagne à chaque répétition qu'il s'en fait. Le sol se garnit peu à peu de trèfle blanc (Trifolium repens, L.), qui troure assez de place pour pousser au loin ses tiges couchées et radicantes et en recourrir peu à peu le champ tout 
entier. Il a pour associé le trèfle rouge (Trifolium pratense, L.), mais celui-ci n'est pas aussi enrahissant que le blanc, et sa souche n'émet pas des tiges allant ramper de tous côtés; il se contente de renforcer son pied et il n'en détache que des rameaux courts. C'est pourquoi le trèfle rouge ne devient jamais aussi prédominant dans un pré que le trèfle blanc.

Mais le purin apporte encore d'autres semences, tant de mauvaises herbes que de bonnes plantes. Les premières toutefois en petite quantité.

Une graminée qui apparaît sourent dans les bonnes terres sans y avoir été semée est le paturin commun (Poa trivialis, L.) Dans les prairies grasses, il se présente à la première coupe en touffes serrées, et, quoique ne s'élerant guère, il ne laisse pas d'être d'un bon rapport. Mais il est plus réduit à la seconde coupe, où il ne fait que ramper sur le sol en prenant la place de plantes plus développées.

Il y a un autre procédé d'engazonnement natnrel, suivi dans certaines régions d'Europe, qui est plus fréquemment employé que celuilà. On commence, il est vrai, par un semis de trèfle; mais, après que, au bout de quelques années, ces plantes se mettent à on disparattre 
laisse à la nature le soin de les remplacer. Nous appellerons cette méthode celle de l'engazonnement naturel perfectionné, pour la distinguer de la précédente, qui est celle de l'engazonnement naturel primitif. C'ependant, ici encore, surtout si l'on arrose de purin sourent et fort, il pousse peu à peu certaines herbes, mais qui ne comptent pas parmi les plus productires ni celles à recom mander. En toutes circonstances, c'est toujours un moyen très coúteux que de rouloir augmenter la production fourragère à force d'engrais, parce que le rendement n'est pas en juste proportion arec la dépense de matières fertilisantes. Sur une terre qui n'est pas déjà garnie de plantes, l'engrais perd son utilité, taudis que, si nous commeuçons par semer les plantes conrenables, les frais de la fumure seront bientót compensés. Il est inexact de soutenir qu'un semis de graminées ne fait pas un engazonnement serré; au contraire, si l'on a recours à un juste choix de graines, l'ensemencement artificiel, arec une dépense égale d'engrais, produit un gazon bien meilieur et un rapport plus considérable.

IV. Semis de graine de fuin

La coutume assez lépandue en Suisse d'ense- 
mencer en graine de foin ne constitue pas un progrès sur l'engazonnement naturel primitif, quoique beaucoup d'agriculteurs pratiques rous soutiennent que ce procédé est encore le meilleur : faut, disent-ils, rendre au sol la semence qu'il a produite bui-méme.

Le moyen rationel le plus sûr de jugger de la raleur de la graine de foin, c'est de l'examiner botaniquement. Des recherches ont été faites et publiées dernièrement daus un Journal d'Agriculture, de Paris. Nous n'en citeroms que deux exemples. Un échantillon de graine de foin passant pour bonne, contenait :

Balles, poussière et impuretés................. 66,52 \%

Graines diverses........................ 33, 38

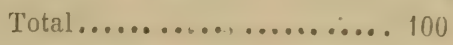

Paturins.......... : 2.89

Houlique liandi-e ... 1.49

Fétuque des prés.... 0.64

Avoine jaunâtre. ... 0.48

I)actyt............ 0.38

Vulpin des prés...... 0.32

Thir-guss anylits... $0.16 \%$ Feturum arine....... 0.16

Flouve odorante..... $0: 16$

Trèfle rouge.......... 0.16

Trelle jaun'........ 0.16

Total des bonnes graines............ 7,14 \% 


\section{$-20-$}

Hlantain lancéolé...... 24.32\%

Brome doux......... 0.48

Crête de coq $\ldots \ldots \ldots . . .60 .32$

Eperviaires.......... 0.32

Oseille............ 016

Boucage à grandes

feuilles........, 0.16

Cumin des prés...... 0.16
Tienontule acre..... $0.12 \%$

Grande fimprelle. 0.12

Myosolis ............ 0.11

Chrysanthème des

moissons....... 0.03

Inconnues $\ldots \ldots \ldots 0.03$

Total des mauvaises herbes.............. 26.34\%

Total général...................... 33.48

Il y a donc prédominence des mauraises plantes, parmi lesquelles on troure le plantain lancéolé pour près d'un quart $(24,320 / 0$.)

Une lirre du mélauge coutenait :

Plantainlancéce 91.934 Report. \& 97936 graines Eperviaires..... 3,060" " f feuilles.... 805 " Cumin des prés.. $965 \quad$ ". Grande pimlueCrête de coq.... 966 " nelle...... 805 "

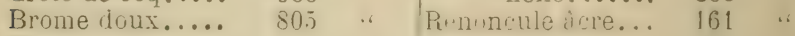
Myosotis....... 805 " Chrysanthème.... 161 "
A reporter... $\overline{97,936}$
Total.... $\overline{910,020}$

Une lirre de cette graine contenait donc 100,029 graines de mauraises herbes.

Celles-ci étaieut fort bien constituées, tandis que les bonnes semences étaient très légères et menues.

$\begin{array}{lr}\text { De l'avoine jaunâtre, il n'a g’ermé que } & 70 / 0 \\ \text { De la houlque laineuse } & 200 / 0\end{array}$ 
Il est facile de comprendre qu'un tel mélange n'est d'aucune valeur pour le culture fourragère et que c'est dommage de sacrifier un seul pied carré du sol à un semis de cette nature.

Il est rrai qu'on peut, comme ça a été fait sourent, nous objecter avec raison que la graine de foin n'a pous toujour's la même composition ; mais il n'est pas vrai de soutenir qu'il peut s'en trouver de bonne qualité, cette semence ne peut être que mauraise, tantôt plus, tantôt moins, et, dans ce dernier cas, le paysan la regarde d'ordinaire comme "bonne". Mais nous venons de faire roir comment est composée cette prétendue bonne graine de foin.

Autre exemple. Un agriculteur, l'un des auditeurs des plus zélés d'un cours sur la culture fourragère, envoya à analyser un paquet de grai. ne de foin. Jusqu'alors, commela plupart des cultivateurs, il avait eu l'habitude de faire une prairie simplement par un semis de graine de foin; mais le peu de raleur de cette semence lui fut démontré au cours de ces leçons, et il fit l'expérience qu'il était plus arantag'eux d'employer un mélange conrenable de graines de graminées et de trè fles pures et capables de germer. Cependant plus tard, ayant considéré combien était belle la graine de foin trouvée au printemps sur son fenil, il eut 
la tentation de retenir à l'ancien procédé, et c'est pourquoi il en expédia un échantillon à examiner, arec la remarque qu'elle ne pourait pas être aussi mauraise qu'il l'arait entendu affrmer dans le cours par lui suiri, et que certainement? elle était meilleure que celle recueillie ordinairement, puisque les prairies d'où elle provenait étaient toutes bonnes et grasses. Il importait donc beaucoup de reohercher la vraie valeur de cette semence et il pourait etre intéressant à d'autres cultirateurs de saroir, une bonne fois, quelle est la composition d'une "bonne graine de foin."

L'analyse a trouvé :

Balles

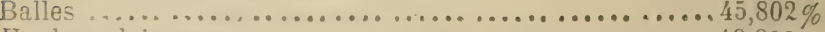

Houlque laineuse............................. 49.909

Autres graines .............................. 4,277

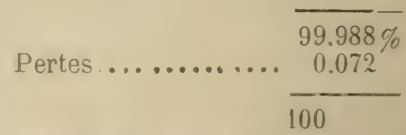

Elle consistait donc à côté des balles, pour la plus grande partie, en graine de houlque laineuse. Or, sur 800 de celles-ci $\$ 7$ seulement g'ermèrent, soit 6o/0, de sorte, qu'en nombre rond, cette graine ne contemil que 3 r.go des dites graines cajables de germer.

Mais il y a d'autres raisons, plus importantes encore, de déconseiller l'emploi de la graine de foin comme semence pour les prairies 
La houlque laineuse n'a ancune raleur : elle doit être regardée absolument comme une mauvaise herbe. D'ailleurs, cette mauraise plante, comme toutes celles yui lui ressemblent, s'introduit d'elle-même dans les prairies, en quantité toujours beaucoup trop forte. Et si, de plus, elle y est encore semée par la main de l'homme, on la rerra pulluler et l'emporter sur toutes les autres plantes meilleures qu'elle, former de gros coussins de gazon et devenir une manvaise herbe des plus nuisibles. Le foin de la houlque laineuse, comme nous l'arous déjà remarqué, étant lég’er comme de la laine, on se tromperait fort en pensant évaluer le rapport d'après le rolume. En outre, la qualité même de ce fourragge est médiocre ; aussi n'est-il pas mangé rolontiers par le bétail, qui probablement ne le dig’ère que d'une manière incomplète.-C'est pourquoi il ne faut attribuer à de telles graines de foin aucune raleur.

Cependant, parmi les autres graines, il y en a aussi environ 20 olo de bonnes, et ce sont:

Dont ont germé

Fétuque des prés .............

Dactyle.

Trèfle rouge.

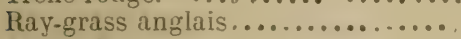

A.utres bonnes semences...........
0,510

0,255

0,127

0,127

0,151
37

7

30

25

25 
La proportion des bonnes graines est donc si minime, et elles sont si peu capables de germer, qu'elles doirent être considérées comme sans valeur. La fleur contenait en outre 0.510 o/o de graines de Lupuline, mais dont aucune n'a germé. Malgré ces résultats défarorables, cette graine de foin compte parmi les moins mauraises, parce qu'elle ne contient proportionnellement qu'un petit nombre de mauraises herbes, soit par livre seulement $26,77 \frac{1}{*}$ grannes des espèces suirantes:

Plantain lancéolé........................

Grande marguerite $\ldots . . \ldots \ldots \ldots \ldots \ldots \ldots$

Crépide

Renoncule âcre ...........................

Grande patience.......................

Petite oseille............................

Oseille des prés.

Aroine jaunàtre. carottu sanvagese, amourette. brome doux, scribe des bujs, etc............

Total des mauvaises graines...
10,710 graines

$4,7 \mathrm{I} 7$

2,805

2,040

1.78 .5

1,020

765

2.932

26,774 par livre.

En semant sur un acre de terre la graine de foin de 20 sacs de 33 lirres, l'on y apporte aussi 18 millions de graines de mauraises herbes: or même en admettant qu'il n'en lère que le quart, l'on sera toujours affligé de $\frac{1}{2}$ million àe pieds de plantain lancéolé, de $\frac{3}{4}$ de million de grande marguerite, de $\frac{1}{2}$ million de crépide, de $\frac{1}{3}$ de millıon de renoncule âcre, etc, etc. Eli rérité, à 
ce compte, le peu de graines de bonnes espèces, capables de germer, sint encore payées beaucoun trop cher!

Tels sont les résultats de l'aualyse d'une graine de foin passant pour "boune" ; on peut se faire une idée de ce que sont les mauraises.

Dans une seule livre d'un mélang’ebien composé de semencesdu commerce, nousarous en graines de bonnes plantes, capabies de germer, autant que dans un quintal de graines de foin, et de plus, par là, nous n'apportons sur le champ aucune des mauraises herbes, tandis que, arec ce quintal de graine de foin, nous semous aussi au moins $2 \frac{1}{2}$ millions de mauvaises herbes, qui compromettront fort la récolte. Ce n'est que grâce à ume semence, pure de mauraises herbes et d'une haute faculté germinative, que nous pourrous tirer de notre terrain le plus fort rendement de fourrage. Et ce fourrage aura d'autant moins de valeur que celle-là sera plus impure et plus mauraise.

Tout le monde pourra se convaincre par des essais que le semis de graine de foin est non seulement d'un rendement fort inférieur, uniais encore de moindre qualité que le produit d'un ınélangye de graminées et de trofles bien appropriés au terrain. 


\section{A. Nowacki a fait comparativement des semis} de graine de foin et de trois différents mélanges, il a obtenu les résultats suirants :

\begin{tabular}{|c|c|c|c|c|}
\hline \multirow[t]{2}{*}{ FoIY PAR ACRE } & \multicolumn{3}{|c|}{ MÈLANGES } & Graine \\
\hline & I & II & III & IV \\
\hline 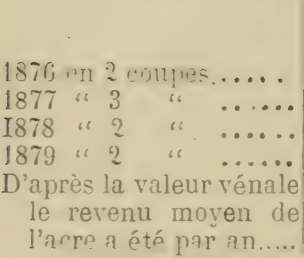 & $\begin{array}{c}\text { Livres } \\
3189 \\
8360 \\
7916 \\
8394\end{array}$ & $\begin{array}{c}\text { Livres } \\
2968 \\
\$ 3: 19 \\
7396 \\
8681\end{array}$ & $\begin{array}{c}\text { Livres } \\
2836 \\
8052 \\
7696 \\
9081\end{array}$ & $\begin{array}{c}\text { Livres } \\
1868 \\
4375 \\
5826 \\
6604\end{array}$ \\
\hline
\end{tabular}

Ce tableau démontre que le rendement des graines fourragères est, non seulement d'un tiers supérieur à celui de la grraine de foin, quant à la quantité. mais que sa raleur rémale lui est aussi supérieure de $\$ 12.73$, à l'acre. De sorte que le rapport de la graine de foin constitue une perte sèche de \$76.38, pour une période de six ans. Cela étant, il est donc évidentqu'il y a tout à gagner de faire, une fois pour toutes, un semis de graines mélangées, lequel coûte environ six piastres à l'acre.

Par conséquent, le semis de graine de foin ne constitue pas un grand progrès sur l'engazonne- 
ment naturel : dans les deux cas, surtout dans les premières années, lo produit est très médiocre, de peu de qualité et mêlé d'innombrables mauraises herbes et de plantes suspectes. Il est clair que la culture fourragère, pratiquée d'après cette néthode, ne peut être remunératrics et n'est pas une culture conforme à nos idées actuelles. Cette manière irrationnelle de produire des fourrages, pouvait se justifier il y a un siècle et plus ; mais elle est absolument contraire aux conditions dans lesquelles nous nous trouvous aujourd'hui, par suite du prix de la terre, de la main-d'œuvre et de la vente des produits. Actuellement, il faut que dès les premières années, la culture fourragère soit d'un plein rapport, si l'agriculteur veut en avoir du bénéfire; et il faut aussi que le fourrage soit exempt, le plus possible, de mauvaises herbes et de plantes de peu de valeur.

\section{v. Théorie et calcul des mélanges}

Pour l'engazonnement artificiel d'un terrain l'on peut suivre deux voles principales: l'une consiste à mettre en semis mur, et seule, telle ou telle plante fourragère; l'autre, à mettre un mélangre de deux ou plusieurs espèces. En semis purs, on emploie généralement le trèfle rouge, le sainfoin, la luzerne ou le fromental. Mais quant à la culture du trèfle, celle-ci ne peut recevoir plus 
d'extension, parce que le trèfle rouge ne réussit d'une manière súre qu'à la condition de ne revenir sur le même champ que tous les huit ans. En outre, cette espèce est d'un produit très incertain. Daprès une expérience de quarante ans, BLock affirme que, même sur un sol très bien approprié à la culture du trèfle rouge, l'on ne peut compter en quatre périodes que sur truis récoltes complètes Ce que BLoch disait il y a ringt-cinq ans, s'applique encore mieux aux circonstances actuelles. La luzerne est excellente comme four. rage vert; mais il faut la faucher à l'épnque où les fleurs commencent à s'ourrir; arant cette époque, la plante est trop aqueuse; après, les tiges en sont trop dures. La luzerne cultivée, la seule rariété qu'il faut semer, réussit très bien daus un sol argileux.

En général, les plantes fourragères cultirées isolément, c'est-à-dire en semis purs d'une seule espèce, ne domnent point les plus forts rendements. mais le priroduit le plus graml, le plus sûr et le plus soutenu ne s'ollient que par le semis de graminées convenables tt de l,oñne qualité, mises en mélange avec des rgumintuses en cie justes proportions. Ces dernières poussent leurs racines dans les plus profondes couchts du sol, pour $y$ puiser, en grande partie, les éléments minéraux et l'eau néct ssaires à leur 
subsistance et à leur accroissement. La souche du trèfle rouge descend à $2 \frac{1}{2}$ pieds; celle de la luzerne à six pieds, et l'on en a même trouvé des racines longues de vingt à trente pieds; celle du sainfoin s'enfonce à vingt pieds et darantage. Les graminées robustes étendent leurs racines dans les couches moyennes du sol régétal, tandis que la couche sapérieure est occupée par celle des graminées fines. Il en est de inême des organes aériens des plantes. Les graminées élevées; telles que le fromental, le dactyle, la fétuque des prés, etc., portent leurs chaumes et leurs feuilles haut dans les airs, pour en utiliser les gaz, la lumière et la chaleur; les graminées de taille moyenne et les légrmineuses tirent leur nourriture atmosphérique de la région intermédiaire; et enfin les graminées et les légumineuses basses virent dans la partie inférieure. De cette façon, on utilise les différentes couches du sol et de l'air de la manière la plus arantageuse, et c'est pour cette raison qu'on obtient le plus grand rendement d'un mélange composé rationnellement. En ne semant qu'une seule espèce, on n'utilisera pas complètement l'air et le sol ; ainsi, par exemple, dans un semis pur de fléole des prés (mil, timolhy), ce ne sont que la couche supérieure du sol et la couche moyenne de l'air qui sont mises à profit, tandis 
que les autres restent sans usage. -Dens cette esquisse nous n'arons admis que trois divisions de la profondeur du sol et de la hauteur de l'eau; mais il serait plus exact de dirisar le sol et l'air ambiant en autant de couches qu'il y a d'espèces végétales rivant à leur dépens, attendu que les plantes d'un mélange bien composé ne se prêtent pas à des séparations aussi précises, mais présentent d'insensibles transitions entre les espèces ; les unes à racines profondes ou superficielles; les autres à taille ibasse ou élerée, plus ou moins.

Les mélanges ont aussi moins à souffrir des influences nuisibles extérieures, telles que l'humidité, la sécheresse, les gelées, les maladies, les insectes, etc.

Si telle plante est compromise par la sécheresse, une autre, qui y résiste mieux, en prend la place; et, au contraire, l'espèce qui ne s'accomode pas d'un excès d'humidité est remplacée par une autre à laquelle elle est favorable. Les mélanges souffrent moins aussi de la cuscute, de l'orobranche, des champig'nons, des insectes. Si certaines de ces plantes en sont attaquées, il en reste d'autres pour remplir le vide qui en résulte. Les g’elées sont aussi moins dangereuses pour les espèces mélangées, parce que les moins sensibles forment un abri pour celles que sont plus délicates. Par conséquent, le 
produit des mélanges est à la fois plus abondant et plus assuré que celui des semis purs.

Mais ce n'est pas seulement sous le rapport physique que les direrses espèces de plantes usent différemment du sol; il en est de même sous le rapport chimique. Les légumineuses lui enlèvent beaucoup plus de magnésie et de chaux que les graminées, tandis que les cendres de celles-ci sont plus riches en silice.

D'après Emile $\Pi$ olff, 1000 parties des plantes suirantes, desséchées à l'air, contienment:

\begin{tabular}{|c|c|c|c|c|}
\hline & Pritasse & Chanux & Mąunési. & silice \\
\hline $\begin{array}{l}\text { Fléole des prés.... } \\
\text { Trifle rouge...... } \\
\text { Trefle alsi } \\
\text { Luzerne........... } \\
\text { Sainfoin......... }\end{array}$ & $\begin{array}{l}7,40 \\
4.40 \\
2.40 \\
4,60 \\
3,40\end{array}$ & $\begin{array}{l}1,60 \\
4,80 \\
3.601 \\
7,90 \\
4,40\end{array}$ & $\begin{array}{l}0,70 \\
1.50 \\
1.10 \\
1,00 \\
0,80\end{array}$ & $\begin{array}{l}7,70 \\
0103 \\
0,411 \\
1,10 \\
1,00\end{array}$ \\
\hline
\end{tabular}

C'est pourquoi, par un mélange de légumineuses et de graminées, le sol étant, chimiquement aussi, utilisé d'une manière plus genérale, est d'autant moins sujet à un épuisement partiel.

Daus un mélange bien entendu, il entre à la fois des plantes précoces et tardives; l'une domne son plus grand rapport à la première coupe, tandis qu'une autre réserve au regain son plus grand 
déreloppement. Il s'ensuit, qu'on a une première et une deuxième coupe de bon rendement.

Telle espèce se déreloppe bien et rend beaucoup déjà, la première année; telle autre n'arrire à sou entier déreloppement que l'année suivante; tandis que d'autres encore ne donnent leur rendement principal que la troisième ou la quatrième anriée. On roit donc qu'un mélang'e composé rationnellement rapporte beaucoup dès la première année et encore les suivantes.

Un fourrage mélangé est aussi plus profitable au bétail que des graminées ou des légumineuses servies séparément. Les dernières, prises pures, causent sourent la météorisation (gonflement du rentre chєz le bétail), ce qui entraîne de grands dommages pour l'agriculteur. Or, il est bien moins exposé à ce danger par l'emploi des mélanges. D'un autre côté, les graminées pures ne sont pas mangées rolontiers par le bétail, qui prendra arec plaisir un mélange de graminées et de légumineuses.-- Un mélange bien composé profite mieux également à la nutrition des bètes. - Il y a cet arantage encore que les légumineuses un peu mélangées de graminées, sèchent plus lacilement et se laissent aussi mieux conserver. Quand, dans un pré ne portant exclusivement que du trè̀le rouge, de la luzerne ou du sainfoin, ces 
plantes ne réussissent plus bien, elles ne cesseront de donner un bon résultat al moyen d'un mélange qui les contient en proportions convenables.

La quantité à employer de graines en mélanges doit se mesurer sur la quantité de celles d'un semis pur fixé par l'expérience et des essais.

Dans le tableau $\mathrm{J}$, colonne $\mathrm{V}$, est indiquée, à l'acre, la quantité moyenne de semence de dixhuit espèces de graines, déterminée d'après les données de nombreux écrivains agricoles et de marchands grainiers, ainsi que d'après les essais et la pratique des principaux agriculteurs de la province.

Les quantités de semence indiquées dans la colonne $\mathrm{V}$ ne doivent pas être prises comme fixes et invariables, mais elles peuvent être modifiées, suivant les circonstances locales. En tenant compte de ces circonstances, tout agriculteur devrait établir à son usage un tableau semblable, pour y noter la composition de ses mélanges. De cette manière, il deviendra, en peu de temps, à même de déterminer les mélanges les mieux appropriés au terrain qu'il exploite. 


\section{TABLEAU I.}

QUAXTITÉ DE SEMEXCE A L'ACRE.

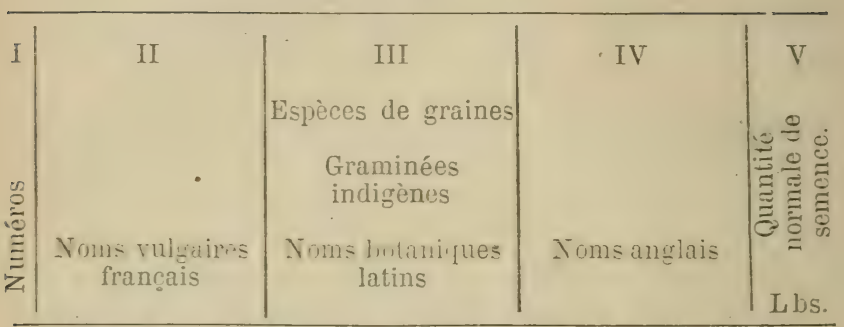

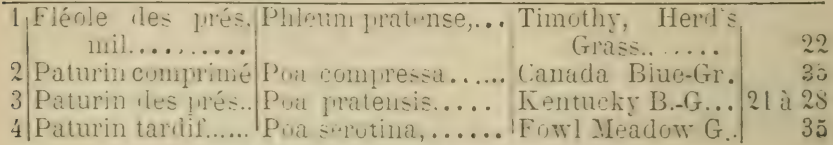
GPAMINÉES ÉTRANGËPES.

5.Avoine élevée,

fromental.... Avena elatior, ..... Tall Oat Grass... $\quad 35$

6 Aroine jaunitre.. Arena flavescens... Jellow Oat Grass, 35

7 Brome inmrne.... Bromus infmis, Austrian Brome G 32

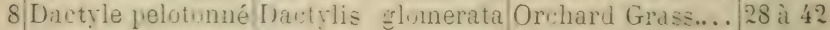

9Fétume dur tte... Festuca luriuscula. Hart Fescue..... 25

10 Fét. les brebis... Festuca ovini..... Shể je Feccue... 25

11 Féiurue élevée... Festuca elatior.... Tall Fescue...... 25

12. Fétuipe des pés.. F. pratensis........ Meadow Fescue.. 35

13 Vulpin des prés... Alopeurus jrat.... Meadum Foxtail.. 25

L EGUMINEUSES.

14 Tr rouge commun Trifibl. pratense,.. . Red-Clorer, Com-

15 mon red Cilov.

16 T. hyl rile, alsique Tri. hybridum.... Alsike Clorer....

Trèlle blanc, T. de

I7 Hullande....

Trifolium rejens... White Clover....

$18 \mathrm{~L}$ uzerne cultivée.. Medicago sativa,... L ucerne Medick.

Sainfoin cultivé... Onobrychis sati.... Hedysarum. 
Nous avons vu précédemment qu'un mélan. ge de graines fourragères produit un plus grand nombre de plantes, sur un espace donné, que si ces mêmes graines eussent été semées séparément. Il faut donc une plus forte semence d'un mélange que d'une seule espèce de graine, mais chaque espèce doit entrer dans le semis pour une moindre quantité.

L'agriculteur intelligent pourra fixer d'une manière rationnelle, la quantité de semence requise pour chaque mélange de graines, en tenant compte des considérations suirantes:

1o Plus le mélange est complexe, c'est-à-dire composé d'un plus grand nombre d'espèces, plus il faut augmenter la quantité de chacume d'elles. S'il ne consiste qu'en deux espèces presque identiques, un supplément n'est pas nécessaire, mais plus le mélange contient d'espèces et plus en est différente la régétation, plus grand doit être aussi le supplément, parce qu'en ce cas, il peut se dérelopper plus de plantes sur un même espace.

20 Plus est médiocre la nature du sol, plus grand doit être le supplément. Il faut le renforcer d'autant plus que le sol est plus différent de ce qu'on entend par "sol normal ou moyen" : pour une terre froide et pesante, ainsi que pour celle qui est légère, il faut p’us de supplément 
que pour une terre chaude et de compacité moyenne.

30 Le taux du supplément se règle en outre sur l'engraissement du sol: cette addition peut être noindre sur un sol bien fumé que sur une terre maigre, parce qu'ici le tallage des plantes est moins fort.

to Le supplément doit aussi être en raison de la préparation du sol. Sur une terre travaillée grossièrement, il se perd plus de graines que sur celle qui l'est finement; par conséquent, la première en exigee une quantité plus forte.

Un champ insuffisamment préparé est moins bien doté d'une quantité de cent lirres que ne l'est arec cinquante une surface égale qui a été bien préparée.

5o Pour une semaille tardive au printemps, et surtout en automne, le supplément doit aussi être plus fort que là où l'on a semé en temps convenable, parce que dans le premier cas la température défarorable fait périr les plantules en plus grande proportion.

6o Pour le supplément l'on tient compte aussi de l'exposition du champ au soleil ; il peut être plus faible que dans les situations froides et élevées.

To Dans les contrées qui reçoivent peu de 
pluie ainsi que dans celles à basse température d'été, il doit être plus fort que dans les régions chaudes et favorablement arrosées d'eau pluviale.

8o Quoique la faculté germinative ait été prise en considération tout d'abord, il faut noter toutefois que, pour l'emploi d'une semence vieille, légère et généralement d'une médiocre faculté germinative, il faut un supplément plus fort que quand il s'agit de graines bien constituées, lourdes, pleines et de germination régulière, car celles qui sont vieilles, légères et à germination indécise fournissent d'ordinaire des plantes débiles, sourent maladives, dont l'existence est délicate et qui succombent au moindre accident. Cependant celles-ci, comme les meilleures, comptent parmi les graines capables de germer. Combien sont plus vigoureuses au contraire, les plantules produites par une semence saine, fraiche, pleine et pesante; combien sont-elles mieux en état de résister aux influences qui les menacent du dehors et plus propres à donuer bientôt des plantes robustes. C'est pourquoi nous donnons toujours la préférence à la bonne marchandise, quand même, à poids égal, la semence pure et capable de germer coûterait plus cher que celle de moindre qualité.

Nous renons d'exposer les principes qui doivent présider à la composition des mélanges de 
graines fourragères, et nous allons maintenant, po ur mieux élucider la question, composrer un tel mélangee. Supposons qu'il s'agisse de faire un pré de trèlles et de graminées sur une boune terre franche ou de glaise, même humide, il nous faudra procéder de la manière suirante :

1o D'abord, choix des plantes appropriées au sol, au mode d'exploitation, etc., ( Tableau I, colonnes II, III et IV).

20 Firation de la proportion dans laquelle on reut que chaque plante soit représentée sur le pré. En cela, naturellement il faudra aroir plus égard aux espècos qui réussissent le mieux sur ce sol qu'à celles qui rieunent moins bien. L'étude de la flore du pays pourra fournir de bonnes indications; mais on ne peut s'y fier qu'en autant qu'elles s'accordent arec l'expérience, et la fin qu'on se propose. (colonne V).

Pour obtenir les résultats insérés dans les tableaux suirants, on détermine d'abord la quantité de graines que l'on se propose de semer par acre de terre, soit 20 मे 26 livres ; puis, prenant en considération la quantité proportionnelle de chaque graine à employer dans l: mélange, on établit le problème suivant : 100: 40 (trèfle rouge) : : 22 (la quantité à semer) : X (ie nombre cherché). 


$$
\begin{aligned}
& -39- \\
& 100: 40:: 22: x \\
& \div 100 \quad \frac{40}{880}-8 \text { livies et } 80 \text { centièmes. }
\end{aligned}
$$

Pour convertir ces 80 centièmes en ouces, on multiplie ce nombre par 16 et on dirise par 100 :

$$
\begin{aligned}
& 80 \\
& \text { x } 16 \\
& 480 \\
& 80 \\
& \div 100 \\
& 1280 \text {-ou égale, } 12 \frac{3}{4} \text { onces. }
\end{aligned}
$$

\begin{tabular}{|c|c|c|c|c|}
\hline 40 \%o trèfle rouge & $40 \times 22-81$ & livres & $12 \frac{3}{4}$ & onces \\
\hline 30 o 0 trèfle alsique & $\overline{30 \times 22}-6$ & " & $9 \frac{2}{3}$ & " \\
\hline 10 o ${ }^{\circ}$ dactyle & $\begin{array}{r}100 \\
10 \times 22-2 \\
\end{array}$ & " & $3 \frac{\mathrm{T}}{5}$ & c. \\
\hline 100 oromental & $10 \times 22-2$ & “ & $3 \frac{\mathrm{r}}{5}$ & " \\
\hline 10 \% fléole des prés & $\begin{array}{r}100 \\
10 \times 22\end{array}-2$ & “ & $3 \frac{1}{5}$ & " \\
\hline 100070 & 22 & “ & & \\
\hline
\end{tabular}

La quantité de trèfle rouge à semer dans le mélauge sera douc de 8 livres, 123 onces.

Ainsi, s'il s'agit de faire un mélaugre de 40 o 0 de trèlle rouge, comme ci-dessus; de 30 o/o de trèfle alsique, de 10 o 0 de dactyle; de 10 o/o de fromental; de 10 o o de fléole des prés (timothy, mil), le calcul se fera simplement de la manière suivante:

Quantité nécessaire à l'acre. 
Exemple de composition et de calcul d'un tel mélange.

\section{TABleau II}

Trèfles et graminées dont la durée est d'un à trois ans.

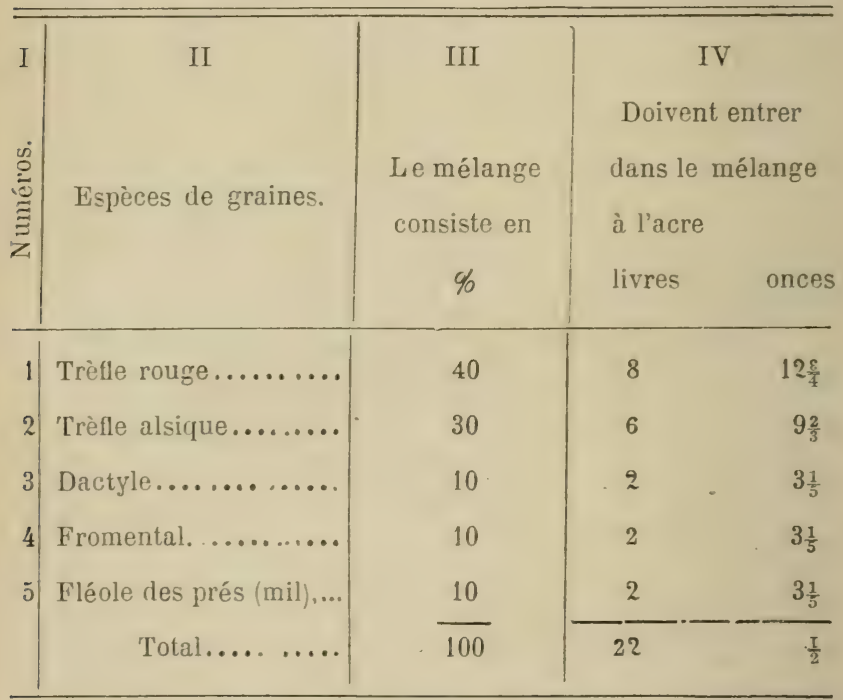

Résumé du tableau précédent.

Trèfle rouge............... livres

Trèfle alsique............. "

Dactyle................ "

Fromental ..............." "

Fléole des prés (mil)............. " "

A l'acre

Ensemble.....

\begin{tabular}{rrrr} 
& \multicolumn{3}{c}{ A l'acre } \\
8 & onces & $12 \frac{3}{4}$ \\
6 & " & $9 \frac{2}{3}$ \\
2 & “ & $3 \frac{1}{5}$ \\
2 & “ & $33 \frac{1}{5}$ \\
2 & “ & $3 \frac{1}{5}$ \\
\hline 22 & & &
\end{tabular}


VI.-Choix des plantes.

Pour composer un mélange dont le rendement soit le plus fort possible, il importe arant tout de déterminer les plantes les mieux appropriées au terrain qu'il s'agit de mettre en prairie. Il y en a qui ne prospèrent que dans un sol légrer, ou compact; d'autres qui en veulent un qui soit sec ou qui soit frais; et elles sont diverses encore par leur durée plus ou moins longue. Toutes ces circonstances doivent être prises en sérieuse considération. C'est pourquoi la composition des mélang'es de semences fourragères doit être fondée sur une exacte connaissance des plantes qu'on y fait entrer. . Par conséquent, il nous faut commencer ici par une étude succincte des principales espèces employées à cet effet.

Les plantes fourragères cultivées jusqu'à présent soit pour être fanées, soit pour servir de pâturage et dont nous nous occuperons ci-après, appartiennent à deux familles bien distinctes, savoir: à la famille des Graminées et à celle des Légumineuses ou Papilionacées. Cette dernière comprend plus spécialement les trèfles, la luzerne, le sainfoin.

A.-Graminées.

Les graminées appartiennent aux monocoty- 
lédones ou plantes à embryon arec un seul cotylédon. Leur tige est ordinairement creuse, ayant des nœuds d'où partent les feuilles, qui sont le plus souvent linéaires et longues. Les contrenœuds varient de quelques lignes à plusieurs pouces. Courts au bas de la tige, ils deviennent plus longs vers le haut. A chaque nœud est insérée une feuille. La partic inférieure de la feuille entoure le chaume comme daus un étui, et s'appelle, à cause de cela, gaine, tandis que la partie supérieure, le limbe, est parfaitement libre. A l'insertion de la gaine au limbe, qui compose la feuille proprement dite, se trouve un prolongement membraneux transparent, nommé ligule, ayant différentes formes et structures, suivant les espèces. Les jets de graminées sortent toujours de l'intérieur de la gaine, des nœuds de la base de chaque chaume, rarement des nœuds supérieurs. La formation de ces jets s'alp pelle le tallage.

Quelques graminées ont un tallage plus fort que d'autres, comme c'est le cas des céréales, qui appartiennent, comme on sait, à la famille des graminées. La force du tallage dépend de la fertilité du sol, de la température et d'autres circonstances. Envertu de cette faculté qu'ont les plantes de taller, un seul grain peut produire un grand nombre de tiges. Les jets se forment à 
l'intérieur de la gaine, soit en perçant celle-ci et en se déreloppant en dehor's perpendiculairement ou en stolons, c'est-à-dire en rampant sous le sol ou à sa surface.

Ces différents caractères sont d'une grande importance au point de vue de la valeur agricole des graminées. Ainsi par exemple, le paturin des prés pousse de longs stolons souterrains, tandis que le paturin commun les a sur terre; et d'autre's espèces, entr'autres le vulpin des prés, ne font que des stolons courts, tandis que la plupart des graminées importantes ne produisent que des jets droits. Si l'on n'emploie à la création des prairies artificielles que des graminées dont les tiges poussent en touffes hautes, on n'obtient pas un gazon consistant et serré ; ces plantes laissent entre elles des rides. En outre, il se forme sourent un gazon à toufles élevées, comme c'est le cas pour le dactyle et pour le fromental. Pour arcir un pré bien constitué, à gazon serré, il faut ajouter aux graminées à touffes compactes des plantes stolonifères et, entr'autres, des petites légumineuses qui donneront de la valeur au fourrage en éloignant la renoncule rampante, le lierre terrestre, la véro. nique à feuilles de lierre, etc.

Nous arons dit plus haut que la tige des graminées ne pousse des jets qu'à sa base. Cela 
ne se rapporte pas à l'inflorescence du sommet de la tige, car beaucoup de graminées ont une ramification à leur sommet, portant des épillets, des fleurs et des semences. Les graminées dont les épillets se trourent directement sur l'axe principal se nomment des graminées à épis; si les épillets sont portés au moyen de pédoncules sur les rameaux du chaume, les graminées sont dites paniculées. Si les rameaux de la panicule sont très courts et très rapprochées, celui-ci ressemble à première vue à un épi et s'appelle, à cause de cela, faux-épi, (fléole, vulpin des prés.)

La fleur des graminées est toujours étroitement enfermée entre les glumelles et les glumes, chez la plupart des espèces s'ourrant au temps de de la floraison.

On désigne cette inflorescence sous le nom d'épillet. Le nombre de fleurs d'un épillet est très rariable. Au centre de la fteur d'un épillet se troure l'oraire, qui porte à soll sommet deux stigmates garnies de papilles. En dehors, sur l'oraire, sont les étamines, au nombre de trois. Celles-ci sont entourées de deux glumelles sourent aristées. Le tout est enfermé dans deux glumes. Fréquemment, les glumes renferment deux et plusieurs fleurs avec glumelles.

La graine des graminées se troure, dans le 
commerce, ordinairement enveloppée des glumelles, et quelquefois même elle est, de plus, renfermée dans les glumes (vulpin des prés.) Ce n'est qu'exceptionnellement qu'on rencońtre dans le commerce de la semence dépouillée des glumelles (le dactyle pelotonné, tout à fait sec, est dans ce cas); cependant, il peut se trourer dans chaque marchandise quelques graius nus. Les graminées ayant des épillets multiflores (le fromental, le dactyle, les fétuques, paturins, brômes, (tc.), conserreut à la base de la glumelle intérieure du fruit mûr, un tronçon de l'axe de l'épillet nommé pédicelle. Le fruit enveloppé des glumelles est mûr. Par une trop grande maturité, le fruit devient corné, tandis que, à l'état frais, la graine rerte est laiteuse à l'intérieur. Les graines peu mûres, zéchées avec soin, penvent encore germer, mais ne produisent que des plantes faibles et maladives. Ces graines sont petites, ratatinées, et perdent bientôt leur faculté gerrninative, tandis qu'une semence parfaitement mûre, grosse et pleine, conserve cette propriété plus longtemps.

Les principales graminées fourragères, dont les diverses espèces ont une valeur plus ou moins grande pour l'agriculture, sont les suivantes. 


\section{. $46-$}

\section{Graminées indigènes}

1. La fléole res prés, mil (Phleum pratense; Timothy, Herd's-Grass).

Le Dr. Gray et plusieurs botanistes américains domnent la fléole comme introduite d'Europe ; cependant Huoker dit l'aroir rencontrée à l'état saurage à Terreneure, sur les bords de la Saskatche. wan et en plusieurs autres endroits de l'Ouest. La fléole prospère surtout daus les terres lourdes, froides et humides, même marécageuses. La variété venant des Montagues-Rocheuses exige un terrain plus sec. La fléole est à bon droit le fourrage le plus estimé, principalement pour la nourriture des cheraux, et c'est aussi celui qui est le plus généralement cultivé en Canda. La tige de la fléole atteint 3 pieds et darantag'e dans les bons terrains. Comme fourrage, on en obtient fréquemment de 300 à 400 bottes de quivze livres à l'acre. On doit toujours la semer en mélange. Il faut $\frac{1}{2}$ minot de semence à l'acre. Cultirée pour sa graine, elle peut en produire jusqu'à 20 minots dans un acre, chaque minot pesant de 40 à 50 lirres.

2.-Le paturin comprimé (Poa compressa; Conad Blue Giass) est une graminée rirace, assez ressemblante au paturin des prés; crpendant il s'eu distingue facilement par ses nom- 
breuses tiges plates et peu élerées. C'est une graminée très rustique, qui résiste à la sécheresse et vient bien dans presque toute espèce de sol. On la rencontre sourent dans les terrains secs et sablonneux, sur le bord des routes, des sentiers, dans les champs arides, sur les coteaux, etc., où la plupart des autres graminées ne pourraient résister. Ainsi, elle convient particulièrement aux endroits rocheux, sablonneux et aux montagnes. Le foin qu'elle fouruit, quoique court, est abondant, très nutritif et très recherché du bétail. Cétte graminée est encore apprériée à raison de sa précocité.

3.-Le paturin des prés (Poa Pratensis; June Grass, Kentucky Blue Grass).

Le paturin des prés est une graminée vivace. Il se rencontre fréquemment dans les pâturages et les prés, il est recherché par tous les animaux. Il fournit un foin d'une bonne qualité, mais il faut le faucher pendant sa floraison; autrement il ne perdrait pas moins d'un quart de sa valeur, surtout si on laissait mûrir sa graine. C'est la plus précoce de toutes les graminées fourragères, sa graine étant sourent mûre vers le 20 ou le 25 juin. Ses racines traçantes et sourent stolonifères lui permettent de résister assez bien à la sécheresse. Il doit toujours être cultiré en mélange. "Nous co nsidérons le paturin des prés comme étant 
indubitablement la meilleure graminée à pâturagge du pays. Si elle est tenue broutée, elle produit d'une manière plus continue qu'aucune autre graminée, une grande quantité de feuilles succulentes depuis le commencement $d u$ printemps jusque tard en automne. I e paturin ou foin bleu du Kentucky est, croyons-nous, une des meilleures herbes, surtout au point de rue de la production du lait. Quand rous jugez bon de labourer rotre pâturage, il laisse une couenne épaisse de bonnes matières fertilisantes. Le paturin des prés, ou foin du Kentucky, doit, à notre avis, former la base de tout mélange pour pâturage." (J. Fletchər.)

Ajoutons ici l'éloge qu'en fait M. I. J. A. Marsan, directeur du collège d'agriculture de l'Assomption. "C'est, dit-il, la plus virace, la plus hâtive et la plus rustique des herbes fourragères; celle qui résiste le mieux aux excès de température, à la sécheresse et aux froids; elle est très nutritice et contient en forte proportion les éléments du beurre et du fromage. C'est cette plante qui influe le plus sur la qualité du beurre et qui lui communique au plus haut degré un arôme remarquable et un goût délicieux d'amande.

"Le paturin est une herbe indigène qui pousse partout dans la Prorince et dans presque 
tous les sols." - Sa semence pèse 13 livres au minot et l'on en compte 244,000 dans une once.

4.-Le paturin tardif des marais (Poa serotina, Fowl Meadow-Grass).

Ce paturin fait un excellent fourrage, plus mou, plus tendre et par conséquent plus facile à presser que la fléole. Il réussit surtout dans les sols bas et humides, et possède le précieux arantage de ne rien perdre de ses qualités, quand bien même on retarde à le faucher ; car, une fois la graine tombée, la tige se couche le plus sourent, et conserre sa vitalité au lieu de se dessécher, émettant de nourelles branches de chacun de ses joints. Ainsi bientôt une seconde végétation succède à la première, et presque aussi abondante. De sorte que ce foin peut être fauché de juillet à octobre, ce qui lui a valu son nom spécifique de tardif.

Il est surtout recherché, comme plus délicat, pour les bœufs, les vaches et les moutons. Lorsqu'il est fauché de bonne heure, il donne, à la fin d'août, un regain presque aussi abondant que la première coupe au commencement de juillet. Son foin est très nutritif. La semence peut donc entrer avec avantage dans les mélanges pour les prairies. Il est vivace ei se rencontre dans les prés humides. 


\section{Graminées étrangères}

5.-L'aroine élerée ou fromental (Avena elatior: Tall Oat Grass.)

Le fromental, ou ray-grass français, rst l'une de nos plus grandes graminées fourragères. Elle peut fournir un fort rendement si elle est cultirée dans une terre qui lui conrienne, c'est-à-dire dans les prés hauts et moyens; car elle redoute un excès d'humidité. Elle est peu rirace, mais sa graine mûrit assez tôt et se ressème, de sorte qu'elle ne disparait jamais entièrement des prairies, une fois qu'on l'a semée. Elle prospère dans tous les sois, même dans les plus ingrats. Elle supporte assez bien la sécheresse.

Le fromental est une graminée à panicule semblable à celle de l'aroine cultirée, arec des tig'es lisses et rides, de 3 à 4 pieds de hauteur. Il fleurit de bonne heure et se durcit rite ; c'est pourquoi on doit le faucher pendant sa floraison. Il fournit alors un foin d'une excellente qualité. Il donne après la fauchaison un regain des mieux fournis. Aussi l'emploie-t-on souvent dans les grands jardins comme le ray-grass d'Angleterre (Lolium perenne), pour former des tapis de rerdure qu'on soumet à la tonte plusieurs fois dans la même saison. Il est donc excellent pour les pâtu- 
rages. Il forme un gazon élargi et peu dense, par suite de son faible tallage, en sorte qu'il ne doit pas être semé seul. Il se développe très vite et donne, dès la première année, un produit considérable ; la deuxième annér, le produit diminue sensiblement, et les plantes commencent à disparattre. Le fromental est donc à sa place dans les prairies temporaires de courte durée.

6. -- L'aroine jaunâtre (Ave na favescens; Yellow Oat Grass).

L'avoine jaunâtre appartient aussi aux meil. leures graminées; elle réussit fort bien dans les terrains chauds. Elle prospère dans les bonnes terres des régions montagneuses, ne supporte pas une grande humidité dans le sol, mais elle est assez résistante à la sécheresse. Les tiges sout bien feuillées et les jets stériles sont très nombreux ce qui en fait un fourrage de bonne qualité. Elle est très recommandée pour les mélanges; elle donne un pâturage très recherché du bétail. Malheureusement, la semence fournie par le commerce est rarement pure ; elle contient presque toujours une plus ou moins grande proportion de dactyle. Cependant on obtient, même arec cette graine, de très bons résultats. Quand on peut s'en procu. rer de la bonne do un prix convenable, on peut recom. 
mander de la faire entrer dans les mélanges en assez forte proportion.

7.-Le brome inerme (Bromus inermis, Austrian Brome Grass).

Le brome inerme est une graminée de Russie extrêmement rustique et de grande raleur ; virace à souche longuement stolonifère (tiges longues) prospérant fort dans les terrains sableux secs et riches en humus. En Hongrie, il se cultive en mélange avec la luzerme. Elle est très hâtive et donne un fort regain. Elle a donné à Ottawa, 104 lirres d'herbe à la rerge carrée, qui s'est réduit par le fanag'e à 47 Jivres d'excellent foin, ou $3 \frac{3}{4}$ tonnes à l'acre.

8.-Le dactyle pelotonné (Dactylis glomerata; Orchard Grass).

Le dactyle pelotonné ou aggloméré est une graminée fourragère des plus précieuses, malgré son apparence ligneuse. Son fort rendement et sa faculté de repousser sans diminuer de rigueur en font un fourrage des plus rustiques.

Le dactyle est une graminée à panicule, mais les épillets nombreux sont rapprochés ou agglomérés en fascicules compactes d'où lui rient son nom. Les feuilles sont longues, larges, consistantes et nombreuses. A la deuxième coupe, les jets 
de feuilles stériles atteignent souvent une longueur de trois pieds, ce qui ajoute la quantité à la qualité. C'est dans la seconde ou la troisième année que le dactyle fournit son plus fort produit, car il se développe assez lentement. Très vigoureux dès sa base, il doune arec le temps des touffes compactes et très saillantes; aussi n'est-il pas propre à être employé seul. Il ne convient qu'en mélange avec d'autres graminées et légumineuses, tant pour prairies permanentes que pour prairies temporaires, si toutefois on veut utiliser ces dernières au moins pendant trois ans.

Le dactyle est très estimé en Angleterre comme fourrage. Il fait une excellente herbe, tant pour prairies que pour pâturages, surtout dans les endroits ombragés. Le meilieur parti à tirer des prairies où il domine, comme on en roit plusieurs sur la côte de Beaupré, serait d'en faire la récolte lorsqu'il est encore vert comme on le pratique en Angleterre. Cette plante croft à peu près naturellement dans tous les terrains.

9. - La fétuque durette (Festuca duriuscula, Hard Fescue).

Herbe petite, mais de grande valeur pour les pâturages élevés, ainsi que sur les sols sablonneux. On la rencontre dans les terrains secs, à St-Joachim, par exemple. 
10. - La fétuque ovine ou des brebis (Festuca ovina, Sheep's Fescue.)

Cette espèce doit son nom spécifique à la qualité et à la finesse de son herbe qui la fait surtout rechercher des moutous. Elle sert avec la précédente à garnir les pâturages, surtout dans les endroits secs, sablonneux et rocheux; leur petite taille ne permet guère d'en former des prairies. C'est une graminée rustique à feuilles filiformes. On la trouve dans les pâturages secs.

11.-La fétuque élerée (Festuca elatior, Tall Fescue).

12. - La fétuque des prés (Festuca pratensis, Meadow Fescue).

Ces deux graminées, introdnites d'Europe, sont viraces et des plus recommandables pour le Canada. Elles sont parfaitement rustiques. Ce sont de hautes herbes, fournissant un foin abondant, très nutritif, de bonne qualité et très estimé du bétail. Elles fournissent aussi une excellente pâture au commencement du printemps et tard en automne. On considère maintenant la fétuque des prés comme étant simplement une rariété de la fétuque élerée. Fille est plus grêle et d'un rendement un peu moins élevé, mais le foin en est plus fin. Il faudrait toujours faire entrer ces graminées nutritives dans les mélanges pour prairies 
et pâturag'es d'assez longue' durée. Ces deux graminées sont bien appropriées aux terrains frais et humides, aussi bien de la plaine que de la montigne. Ces deux fétuques ne sont cultivées qu'en mélang'e. Les tiges ont jusque trois pieds de hauteur; l'inflorescence est en panicule rameuse. Les feuilles sont longues et assez larges, finement striées. Ces herbes se développent rite et donnent, déjà la première année, un bon rapport. Au printemps, elles commencent à régéter de bonne heure, surtout la fétuque des prés; tandis que la fétuque élerée fait mieux à l'automne. Elles croissent promptement, de sorte que, si la terre est bonne, on peut en faire deux coupes dans la même année. La graine de chacune de ces fétuques pèse 14 livres au minot, et l'once en contient 26,000. On trouve ces deux herbes dans les prés, les pâturages.

13. - Le rulpin des prés (Alopecurus pratensis Meudow Foxtail).

Le vulpin des prés est assez semblable à la fléole des prés (timothy), sinon qu'il a l'épi un peu plus court et doux au toucher et que sa fleur n'a qu'une glumelle. La semence ne se séparant pas des balles (ce qui est beaucoup mieux, car l'on ne devrait jamais semer de graine dépouillée de son enveloppe), ne pèse guère plus de cinq livres au mi- 
not, et cependant une once n'en contient pas moins de 76,000. C'est, arec le paturin des prés (Poa, pratensis) la plus hâtive de toutes les graminées, il épie de deux à quatre semaines plus tôt que le fromental. Le rulpin des prés ne produit pas une grande abondance de foin, mais ce foin constitue un excellent pâturage en raison de sa précocité et de la rapidité arec laquelle il repousse après aroir été fauché ou brouté, ce qui lui permet de donner un riche regain. Malgré sa croissance assez rapide dans l'année de la semaille, ce n'est que dans la troisième année que la plante acquiert son entier développement. Il forme un gazon assez serré et pousse des stolons rampant sur un court espace. Les tiges peurent atteindre une hauteur de trois pieds. Beaucoup de graines sont déjà mûres arant la fenaison et se ressèment d'elles-mêmes. Le vulpin des prés cst une précieuse graminée dans les terrains argileux et humides. C'est surtout dans les endroits froids et élevés qu'il donne d'excellents résultats. Il supporte un climat rigoureux mieux que n'importe quelle autre graminée. Il est rira. ce et on Je rencontre dans les lieux froids et élevés, dans les prés, les pâturages.

$$
\text { B.-Lègumineuses }
$$

Les légumineuses ont une structure entière- 
ment différente de celle des graminées. Leurs racines principales pivotent aussi profondément que possible dans le sous-sol (racines pivotantes). Ces racines sont elles-mêmes garnies sur toute leur longueur de radicelles fibreuses. Les racines se ramifient et sont, pour la plupart, droites (trèfle rouge, luzerne, sainfoin), ascendantes ou couchées (trèfle alsique), ou rampantes (trèfle blanc). Les feuilles se composent de trois folioles (trèfle rouge, trèfle alsique, trèfle blanc, luzerne), ou sont taillées, c'est-à-dire composées de plus de trois folioles (sainfoin). Les légumineuses appartienuent à la famille des papilionacées. Chaque fleur est composée de quatre pétales irréguliers; le plus grand, dirigé vers le haut, est nommé l'étendard, le deuxième en grandeur, qui occupe le bas, est dit la carène, et ceux des deux côtés sont les ailes. Les étamines sont au nombre de dix; le plus souvent, neuf d'entre elles sont soudées en un seul tube fendu à sa partie supérieure et renfermant le pistil. Les fleurs sont réunies soit en capitules soit en grappes. Le fruit est une gousse contenant une ou plusieurs graines. Cette gousse est ordinairementéliminée par le battage et l'on en obtient la graine nue (il en est ainsi pour le trèfle rouge et la luzerne), ou bien la gousse entoure 
encore la graine après le battage, comme au sainfoin.

Toutes les légumineuses fourragères en usage dansla province ont été introduites d'Europe et naturalisées ici

14.-Le trèfle rouge.

De tous les trètles, le plus estimé comme fourrage. Il en existe deux variétés: le petit trèfle rouge ordinaire ou commun qui convient mieux pour pâturage et le grand trèfle rouge qui est en général consommé à l'état rert ou comme fourrage sec. C'est dans les sols marneux, ainsi que dans tout terrain d'une certaine consistance, contenant du calcaire, que le trèfle réussit le mieux. Il peut même se mettre sur l'argile la plus compacte, à condition qu'elle soit en bon état de culture; mais il ne s'accomode pas d'un terrain retenant l'eau trop longtemps, de même qu'il ne crôt qu'arec peiue dans les terres trop légères et sablonneuses.

Il est plus sensible au climat que les graminées, et comme tous les trèfles il supporte bien la sécheresse. C'est pourquuoi, dans les années sè. ches, nous royons sur nos prés les graminées peu apparentes, tandis que les trèfles prenuent le dessus. Dans les années farorables il se déreloppe déjà assez la première année pour domner, en automne, une petite coupe. L'année suivante, il 
donne au moins denx coupes, puis en automne on le retourne. Le trèfle rouge n'étant que bisannuel, ne doit entrer que dans les mélanges pour prairie de courte durée.

15.-Le trèfle alsique doit être semé de préférence dans les terres franches ou formées de glaises fraiches et même humides. Il est beaucoup moins sensible que le trèfle rouge et fournit un bou rendement. Plus tardif, il ne se dureit pas aussi facilement que ce dernier. Les tiges du trèfle alsique sont ascendantes; d'abord coudées à leur base, elles se redressent peu à peu. Ses fleurs en capitules sout blanches au centre et roses couleur chair en dehors. Il se conserve mieux comme foin que les autres espèces de trèfle. Il est vivace.

15.-Le trèfle blanc est une plante qui n'a de Taleur qu'en mélange avec d'autres trèfles et graminées, comme herbe basse. Cultivé seul, le produit en serait médiocre. Dans un mélangee, les tiges rampantes du trèfle blanc poussent et s'enracinent partout où elles trourent une place vide. il constitue de cette manière un fond de première qualité pour les pâturages. Le trèlle blanc dure de trois à quatre ans en semis purs, mais en mélange, il est virace et ne disparait jamais complètement. En fumant avec des cendres de bois 
ou de l'ellograjs minéral un vieux pré dans lequel le trèfle blanc est à peine visible, on verra bientôt ce fourrage se développer vigoureusement; ce qui prorient de ce que la plante est mise tout à coup daus des conditions favorables à sa végétation. Mais c'est surtout l'arrosage au purin qui est pour le trèfle blanc d'une efficacité sırprenante. Par cette opération,le sol recoit non seulement l'engrais mais sourent encore une assez grande quantité de graines de cette même plante prorenant du fourrage donné au bétail, lesquelles germent et se développent sur le pré.

Le résidu sec d'un engrais liquide de ce genre ayant été examiné botaniquement, il a été trouvé $q u$ une lirre contenait environ six mille graines de trèfle blanc. Cette légumineuse se trouve partout dans les terrains riches.

La luzerue vient dans notre province pourvu qu'on lui domne une terre bien profonde, riche en principes minéraux (chaux, acide phosphorique, potasse), et un sous-sol perméable et bien drainé. Elle a été cultivée à la Ferme expérimentale d'Ottawa, où elle a donné annuellement trois récoltes d'un excellent fourrage. Dans les montagnes à climat humide, elle est sujette non seulement à être étouffée par les mauvaises herbes, mais l'humidité lui nuit aussi directement. D'après la 
nature $d u$ sol et du climat sa durée varie de trois à vingt ans. Elle ne doit entrer que dans les mélanges qui sont coupés trois fois par an ; sans cela, elle devient dure.

18. - Le sainfoin cultivé est une plante rustique, qui se rencontre dans les pâturages jusqu'à une grande élévation au-dessus du niveau de la mer.

Le sainfoin est un fourrage de premier ordre s'il provient de graines sûres.

Le sainfoin demande avant tout un sous-sol calcaire parfaitement sain ; à ces conditions il réussira, même dans les terres légères, soit sablonneuses ou graveleuses. Comme la jeune plante craint plutôt la sécheresse que le froid, on sème de bonne heure. La graine et son enveloppe doivent avoir une teinte brun-clair. Une innovation consiste à livrer la graine décortiquée, c'est une mauvaise opération.

Pour le faucher, il faut se souvenir que ses feuilles sont d'autant moins adhérentes qu'elles sont plus humides. Son foin quoique un peu grossier, est de première qualité lorsqu'il a été fait dans de bonnes conditions. Son regain n'a pas de rival pour la production du lait.

VII. Mélanges de graines fourragìres.

Nous ${ }_{\mathrm{B}}^{\text {¿ }} \mathrm{les}$ divisons suivant la composition, l'emploi agricole et la durée du rapport en ; 
1o Mélanges de trèfles et de graminées.

20 Mélanges pour prairies temporaires.

30 Mélanges pour prairies permanentes.

\section{1o Trèfles et graminécs.}

(durée de 2 à 3 ans)

Nous comprenons daus cette elasso les mélanges où le trèfle domine ou bien est dans la proportion d'au moins 40 0/0. Comme d'ordinaire la régé tation de cette plante dure peu, le temps qu'un tel mélange reste en bon rapport est senlement de 2 à 3 ans. - Mais il est préférable au semis de trèfle pur, parce que la réussite en est plus assurée et qu'il donne un fourrage aree lequel le bétail est moins exposé à la météorisation (enflure générale de l'abdomen, chez les ruminants, raches, etc., due à des gaz qui s'y trourent accumulés). Un champ qui a porté du trèfle il y a peu d'années, n'est point propre à en receroir de noureau : on risquerait de n'en aroir que peu de profit, tandis qu'il sera consiclérable par le moyen d'un mélange de trèfle et de graminées. A cet effet, on associe à la légumineuse des graminées bien appropriées au sol. Quand celui-ci est trop médiocre et que le trèfle serait sujet à ne pas bien réussir, on le sème également en mélange arec des graminées, telles que la fléole des prés (mil, timothy), pour une terre forte, et le paturin des 
prés, pour une terre plus légère. Le trèfle roug'e peut, en partie, être remplacé aussi par d'autres espèces de cette légumineuse; par le trèfle alsique, par exemple, pour un sol compact; quand il s'agit d'un pacage uniquement, c'est le trèfle blanc qui doit dominer. Le sainfoin et la luzerne entrent rarement daus la composition des mélanges en question.

Les graminées qu'on y emploie le plu sourent sont : le fromental ou aroine élevée, la fléole des prés (mil, timothy), le dactyle, le paturin des prés.

Les tableatux suivants sont donnés comme exemples de mélangres de trèfles et de graminées, le lecteur ne devant pas perdre de rue la manière de procéder que nous arons indiquée aux pages 38 , 39 et 40. Cependant, dans ces tableaux, nous n'arous inscrit que les résultats obtenus au moyen de ces calculs. En outre, afin d'être plus praliques et pour exempter au cultirateur la peine et la perte de temps qu'exige un semblable travail, nous avons éliminé de la compesition de ces mélanges toutes les quantités moindres qu'une demi-livre.

Voici les tableaux en question: 
A.-Mélanges de deux espèces.

\begin{tabular}{|ll|r|}
\hline \multicolumn{1}{|c|}{ Espèces de graines. } & \\
& Quantité de semence â l'acre. \\
& Livres \\
\hline Trèfle rouge. & $19 \frac{1}{2}$ \\
\hline Fromental (avoine élevée). & $2 \frac{1}{2}$ \\
\hline
\end{tabular}

Mélanges destinés à une bonne terre.-Plus le sol est léger, plus on reuforce la dose de fromental mais ce n'est qu'exceptionnellement qu'il s'en met pius de 33 o/o. La durée est de deur années.

\begin{tabular}{|l|r|}
\hline II & \\
Espèces de graines. & Quantité de semence à. l'acre. \\
& Li vires \\
\hline Trèfle rouge. & $19 \frac{1}{2}$ \\
Fléole des prés (timothy). & $2 \frac{1}{2}$ \\
\hline
\end{tabular}


Si le mélange de trèlle rouge et cie fromental est mieux approprié à un sol léger et chand, ce deuxième mélange rếussit mieux sur une terre froide et forte, notamment pour fourrage rert. Plus la terre est forte, plus on met de fléole, mais en ne dépassant jo o/o qu'exceptionnelle. ment.

\begin{tabular}{|l|c|}
\hline III Espèces de graines & Quantité de semence à l'acre \\
\hline Lrèfle alsique. & $14 \frac{1}{2}$ \\
Fléole des prés (timothy). & \\
\hline
\end{tabular}

Mélange destiné à une terre humide et froide ou le trèfle est d'un rapport incertain.

Nota.--Un mélange de sainfoin et de fromental n'est pas à recommander, parce que la semence en est chère et que le rendement serait faible et de peu de durée.

\begin{tabular}{|l|c|}
\hline \multicolumn{1}{|c|}{ Espèces de graines } & $\begin{array}{c}\text { Quantité lle semence à l'acre } \\
- \\
\text { Lirres }\end{array}$ \\
\hline Dactỵle & 20 \\
Trètle rouge & 6 \\
\hline
\end{tabular}


Mélange convenant à tous les terrains, mais préférablement pour les lieux ombragés. Ce mélange fait bien surtout pour fourrage vert.

\section{B.-Mélanges de trois espèces.}

\begin{tabular}{|c|c|}
\hline V Espèces de graines & $\begin{array}{l}\text { Quantité de semence à l'acre } \\
\text { Lîrre- }\end{array}$ \\
\hline $\begin{array}{l}\text { Trètle rouge } \\
\text { Trêfle alsique } \\
\text { Flenlu du's prés timmithy, }\end{array}$ & $\begin{array}{l}6 \frac{1}{2} \\
7 \\
3 \frac{1}{2}\end{array}$ \\
\hline
\end{tabular}

Combinaison des mélanges II et III appropriée plutôt aux terres fortes. Plus ces terres sont compactes, plus ont réduit la proportion du trèfle rouge.

\begin{tabular}{|l|c|}
\hline \multicolumn{1}{|c|}{ VI Espèces de graines } & $\begin{array}{c}\text { Quantité de semence à l'acre } \\
\text { Livres }\end{array}$ \\
\hline $\begin{array}{l}\text { Trèlle rouge } \\
\text { Fromental (avoine élevée) }\end{array}$ & $19 \frac{1}{2}$ \\
Fléole des prés & $2^{\frac{1}{2}}$ \\
& \\
\hline
\end{tabular}

Combinaison des mélanges I et II pour une bonne terre de pesanteur moyenue : durép de 2 à 3 ans. 
VII

Espèces de graines

Quantité de semence à l'acre

Livres

Trèlle alsique

Trètle blanc

Paturin des jrés

5
15
5

Mélange ne convenant que pour pâturagee.

$$
\text { C.-Mélanges de quatre espèces. }
$$

VIII

- Espèces de graines

Trèlle rouge

Trèlle alsique

Fromental

Fléole des prés
Quantité de semence à l'acre

Livres

Combinaison des mélang'es V et VI, appropriée à une terre de pesanteur moyenme; pour une terre plus forte on remplace le fromental par la fétuque des prés ou le rulpin des prés (pour les terrains très argileux et humides). Pour pâture, il convient de prendre les trèfles alsique et blanc, le paturiu. des prés et le dactyle. 
IX

Espèces de graines

Quantité de semence à l'acre

Livres

Trèlle rougre ordinaire

Trèlle aissique

Trefle blanc

Dactyle jelotonné

$10 \frac{1}{\frac{1}{2}}$
6
$8^{\frac{2}{\frac{1}{2}}}$

Ce mélange fait bien daus à peu près tous les sols, mais ciest surtout dans un bon terrain sablo-argileux qu'il pousse arec toute sa rigueur. Il convient pour foin, pâturage et fourrage vert.

\section{D. -Mélange de cinq espèces}

\begin{tabular}{|c|c|}
\hline I Espèces fle graines & $\begin{array}{l}\text { (Mantite le somence in lacer } \\
\text { Livres }\end{array}$ \\
\hline $\begin{array}{l}\text { Trèfle rouge } \\
\text { Trèfle alsi(rue } \\
\text { Fromental } \\
\text { Dactỹle } \\
\text { Fléole des prés }\end{array}$ & $\begin{array}{l}10 \\
8 \\
2 \\
2 \\
2 \frac{1}{2} \\
2 \\
2 \\
2\end{array}$ \\
\hline
\end{tabular}

Mélange approprié à nne terre de limon ou de bonne argile; durée de 3 ans.

On roit que les mélanges de cette catégorie ne sont pas de composition bien complexe, et il serait facile d'en ajouter quelques douzaines, mais 
il est impossible de domner, dans un ourrage aussi restreint, des compositions propres à tous les terrains: aussi, celles que nous présentons ici sont moins des recettes que de simples exemples, propres à gruider le cultivateur dans ses calculs en cette matière.

Des mélanges de trèlles et de graminées on obtient généralement un rapport moindre que des prairies temporaires, mais en reranche la semence en est moins chère. D'un autre sôté, ils fournissent un fourrage plus propre à être comsommé en vert que celui des prairies, parce que la proportion des trèfles l'emporte sur celle des graminées. A u contraire, l'herbe des prairies temporaires se prête mieux à être convertie en foin.

Lorsque, dans un tel mélange. les trèfles entrent pour moins de 50 o/0, il doit s'appeler plutôt mélange de graminces et de trèfles. Il s'emploie șà et là des compositions de cette sorte, mais il ne faut pas les confondre arec celles pour les prairies temporaires ou permanentes, qui, comme on le rerra plus loin, sont fondées sur d'autres principes. Dans les mélanges de graminées et de trèfles, il n'entre d'ordinaire qu'un très petit nombre de graminées à durée assez courte.

Il arrive aussi qu'on sème différents trèfles ou d'autres légumineuses, sans addition de graminées, 
comme, par exemple, le trèfle rouge et le trèfle alsique ou le sainfoin, ce qui pourrait s'appeler mélanges de trêfles. Inversement, les mélanges de gruminées ne consistent qu'en espèces de cette famille de plantes, sans addition de légumineuses. Mais ces derniers ne sont pas à recommander, parce que le rendement et la qualité sont toujours médiocres.

20 Milange; pour prairies temporaires (Durée de 3 à 6 ans).

Ces mélanges sont destinés principalement à la culture intensire, sur les terres de bonne qualité et bien fumées. Dans ce cas leur rendement est sourent énorme. Il ne peut être égalé ni par les prairies permanentes ni par les trefles et graminées. Il importe de les composer d'après les principes suivants.

1. Les légumineuses ne doirent y entrer en général que pour un tiers $(3.30 / 0)$, si l'on reut que la culture soit dans les meilleures conditions de rapport et de durée.

2. Il faut mettre à la fois des herbes hautes, moyennes et basses.

3. Les espèces les plus durables, telles que le dactyle, la fétuque des prés et le paturin des prés, doirent s'y trouver dans les proportions conrenables. 
Contrairement aux mélanges de trèfles et de graminées, ceux dont' il s'agit ici ont l'avantage de rester plus longtemps en rapport, d'être généralement d'un rendement plus fort et surtout plus sûr; mais les prés coûtent un peu plus cher à établir et il fant plus de savoir et d'expérience pour composer un mélange qui leur convienne Les prairies temporaires l'emportent sur les permanentes en ce qu'elles coûtent moins cher à mettre en valeur et sont plus productives.

La durée d'exploitation d'une prairie temporaire bien conditionnée dépend de l'état du sol, de sa nature plus ou moins compacte, de son degré d'humidité, de son état de fumure et aussi de ce qu'on peut lui fournir d'engrais dans la suite. Cette durée sera d'autant plus longue que le sol est plus compact et plus propice à la production fourragère, quand d'ailleurs les autres conditions sont également farorables. Si pendant l'exploitation on ne peut faire annuellement un arrosage au purin, la régétation sera moins plantureuse déjà la quatrième année; il faudra alors sans retard rompre le pré, en ameublir le sol et le soussol, fumer de nouveau, pour le remettre en culture, ce qui permettra d'en tirer diverses récoltes pendant une ou plusieurs années, suivant les circoustances; après quoi on y fera un nouveau 
semis de plantes fourragires. Il raut mieux, en général, procéder ainsi, que d’aroir recours à un semis supplémentaire. Il est rrai que le terrain, après aroir été labouré, peut être remis en pré immédiatement, mais il est préférable de le faire porter d'abord une récolte intermédiaire.

Maintenant, roici quelques exemples de mélanges pour prairies temporaires Nous arous pris pour base de nos mélanges la quantité de 23 à 2.5 lirres de graines, suirant l'espèce de sol comme étant la meilleure proportion pour le climat de notre Prorince.

A. - Mélunge mori une bonne terre ferlile, riche on humus, de lim'm, de marne limoneuse. ou de marne argileuse, avec sous-sol sablonneux.

\begin{tabular}{|c|c|c|}
\hline & Espèces de graines & $\begin{array}{c}\text { Quantité de semence à l'acre } \\
\text { Livres }\end{array}$ \\
\hline $\begin{array}{r}1 \\
2 \\
3 \\
4 \\
5 \\
6 \\
7 \\
8 \\
9 \\
11\end{array}$ & $\begin{array}{l}\text { Luzerne } \\
\text { Trèle rouge } \\
\text { Trèfle blanc } \\
\text { Trèfle alsigue } \\
\text { Fromental } \\
\text { Fétuque élevée } \\
\text { Fétuque des prés } \\
\text { Paturin des prés } \\
\text { Dactyle } \\
\text { Fléole des prés }\end{array}$ & $\begin{array}{cc}1 & 1 \\
2 & 1 \\
1 \\
3 \\
2 \\
1 \\
3 \\
3 \\
3 \\
2 \\
3 & \frac{1}{2} \\
3 & \frac{1}{2} \\
24\end{array}$ \\
\hline
\end{tabular}


Où la luzerne risque de ne pas réussir, on peut la remplacer par une autre léguminense.

B.-Mélunge pour une bonne terre argileuse, riche en humus

\begin{tabular}{|c|c|c|}
\hline & Espèces de graines & $\begin{array}{l}\text { Quantité de semence à l'acre } \\
\text { Livres }\end{array}$ \\
\hline $\begin{array}{l}1 \\
2 \\
3 \\
4 \\
5 \\
6 \\
7 \\
8 \\
9\end{array}$ & $\begin{array}{l}\text { Trèfle rouge } \\
\text { Trèfle blanc } \\
\text { Trèlle alsique } \\
\text { Fétuque des prés } \\
\text { Fétuque élevéc } \\
\text { Dactyle } \\
\text { Vulpin des prés } \\
\text { Fléole des prés } \\
\text { Paturin tardif }\end{array}$ & $\begin{array}{cc}3 & 1 \\
1 & \\
3 & \\
2 & 1 \\
1 & 1 \\
2 & 1 \\
2 & 1 \\
2 & 1 \\
4 & 1 \\
2 & \frac{1}{2} \\
2 & \end{array}$ \\
\hline
\end{tabular}

1. Plus la terre est forte, plus le trèfle rouge peut être remplacé par le trèfle alsique.

2. La fléole des prés et le dactyle sont les types des plantes propres aux terres fortes, et c'est la fléole des prés qui conrient le mieux pour ètre substituée à une espèce qu'on roudrait laisser de côté. 
C.-Mélange pour une terre de sable limoneux, profond et riche en humus.

\begin{tabular}{|c|c|c|}
\hline & Espèces de graines & Quantité de semence à l'acre \\
\hline \multirow[t]{2}{*}{$\begin{array}{r}4 \\
5 \\
6 \\
7 \\
8 \\
9 \\
10\end{array}$} & $\begin{array}{l}\text { Luzerne } \\
\text { Trềle rouge } \\
\text { Trêfle alsique } \\
\text { Fromental } \\
\text { Fétuque élevée } \\
\text { Fétuque des prés } \\
\text { Dactyle } \\
\text { Fléole des prés } \\
\text { Paturin des prés } \\
\text { Brome inerme }\end{array}$ & $\begin{array}{ll}2 & \frac{1}{2} \\
3 & \\
2 & \frac{1}{2} \\
3 & \frac{1}{2} \\
1 & \\
2 & \frac{1}{2} \\
3 & \\
1 & \\
3 & \\
2 & 2\end{array}$ \\
\hline & & $\overline{24}$ \\
\hline
\end{tabular}

1. Si l'on reut substituer une espèce de graminée à une autre, le dactyle mérite la préférence.

2. Où la terre n'est pas assez profonde pour la réussite de la luzerne, il faudra une légumineuse mieux appropriée.

D. - Mélange pour une bonne et profonde terre calcaire marno-calcaire on marno-sablonneuse.

Espèces de graines

Quantité de semence à l'acre

Livres

1 Sainfoin

2 Luzerne

3 Trifle blanc

4 Fromental

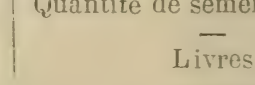


5 Dactyle

6 Fléole des prés

7 Paturin des prés

8 Brome inerme

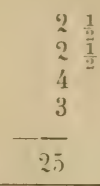

1. Les méianges où le sain foin domine sont toujours chers, et le rapport en est disproportiouné à la dépense; partout où c'est possible, il faut chercher à le remplacer, jusqu'à concurrence de moitié, par du trèfle blanc et rouge.

2. Là où le sainfoin réussit sùrement, le fromental convient aussi.

E.-Mélange pour une bonne terre humeuse, drainée et accessible au voiturage de fumier et de marne.

\begin{tabular}{|ll|c|}
\hline \multicolumn{2}{|c|}{ Espèces de graines } & Quantité de semence à lacre \\
Livres
\end{tabular}


Suirant la mature du sol, le trèfle alsique, le dactyle et la fléole des prés tiennent ici le premier rang.

F-Mélange pour une terre argileuse, humide tt froide.

\begin{tabular}{|c|c|c|}
\hline & Espèces de graines & $\begin{array}{c}\text { Quantité de semence à l'acre } \\
\text { Livres }\end{array}$ \\
\hline $\begin{array}{l}1 \\
2 \\
3 \\
4 \\
5 \\
6\end{array}$ & $\begin{array}{l}\text { Trèlle rouge } \\
\text { Trèfle blanc } \\
\text { Trèfle alsique } \\
\text { Dactyle } \\
\text { Fléole des jrés } \\
\text { Vulpin des prés } \\
\text { Fétuque des prés } \\
\text { Paturin tardif }\end{array}$ & 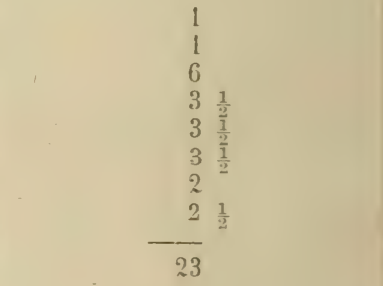 \\
\hline \multicolumn{3}{|c|}{$\begin{array}{l}\text { G.-Délange poui sol argileux (glaiseux), humide et } \\
\text { de quolité moyenne, aver sous-sol perméable. } \\
\text { (M. J. Fletcher). }\end{array}$} \\
\hline & Espèces de graines & Quantité dle semence ì l'acre \\
\hline $\begin{array}{l}1 \\
2 \\
3 \\
4 \\
4 \\
5 \\
6\end{array}$ & $\begin{array}{l}\text { Luzerne } \\
\text { Trèfle rouge commun } \\
\text { Trèfle alsique } \\
\text { Trèfie blanc, de Hollande } \\
\text { Paturin des prés } \\
\text { Fléole des prés (mil) }\end{array}$ & $\begin{array}{l}2 \\
2 \\
2 \\
2 \\
1 \\
6\end{array}$ \\
\hline
\end{tabular}


7 Fétuque des prés

8 Dactyle pelotonné

3o. Mélunges pour les pratries permanentes ou naturelles.

(Durée de plus de 6 ans)

Lorsqu'il s'agit d'une prairie derant rester un peu longtemps en exploitation, il faut procéder d'une manière particulière. La proportion des légumineuses ne doit pas dépasser 20 olo. Laa proportion des plantes de per de durée, comme le trèfle rouge, le fromental, et la fléole des prés, doit être réduite plus encore que pour les prairies temporaires, tandis qu'il importe d'aroir recours davantage aux herbes, légumineuses et graminées, de longue durée. Les graminées sus mentionnées étant généralement moins productires, on n'obtient pas de ces mélanges un rendement aussi fort que de ceux des deux catégories précédeutes, parce que, pour prolonger l'exploitation, on y domne moins de place aux espèces d'un grand rapport. C'est pourquoi, partout où il est possible, une culture fourragère bien entendue doit donner la préférence, soit anx mélanges de trèlles et de graminées, soit, ce qui vaut mieux que tout, à ceux 
pour prairies temporaires, et ne mettre en prairie permanente que dans les cas où il est impossible ou défarorable de faire autrement. Ces cas sont les suirants.

1. Terrains exposés à des inondations;

2. Pentes trop fortes, où la rupture fréquente du pré ne peut aroir lieu facilement;

3. Terres excessirement compactes et résistantes, qui sont difficiles à travailler, et ne donnent que peu de rapport à la culture des céréales, ou, au contraire, sont d'un sol par trop léger.

4. Hauteurs où les conditions météorologigues sont contraires aux céréales et farorables aux plantes fourragères;

ว. Endroits propres à la formation de prairies irriguées (arrosées);

6. Lieux roisins de la ferme et vergers plantés de beaucoup d'arbres, ne se prêtant point à être rompus fréquemment, mais propres à faire des prairies permanentes, dont le rendement peut derenir très fort, grâce à de copieux arrosages de purin ;

7. Champs trop éloignés de la forme et en géuéral toutes terres ne se recommandunt pas pour une culture intensive, comme, par exemple, celles 
qui sont trop pénétrées d'eau, ou trop sèches, ou trop maigres.

Du moment qu'une prairie naturelle (permanente), infestée de mauraises herbes et de plantes médiocres prenant la place des bonnes, n'est plus que d'un faible rapport, il ra de soi qu'en toute circonstance où c'est possible, il faut la rompre et ensemencer de noureau. Si l'on peut faire de la culture intensive, on utilisera dorénavant le terrain soit comme prairie temporaire, soit en y mettant un mélange de trèfles et de graminées; sinon, l'on en fera de noureau une prairie permanente.-Quand il faut acheter la semence, le coût d'un mélange pour prairie permanente est encore plus élevé que pour une temporaire, parce que les graines des graminées durables sout les plus chères. L'établissement d'une telle prairie et le choix de ses plantes exigent de sérieuses réflexions et l'attention la plus minutieuse, parce que de là dépend arant tout le rendement que nous en espérons pendant de longues années. C'est pourquoi une connaissance approfondie de la matière et beaucoup d'expérience agricole sont ici plus nécessaires encore que quand il s'agit d'une prairie temporaire. 
Toici quelques exemples de mélanges pour prairies permanentes:

H.--Mélunge pour une bonne terre de limon, de marne limoneuse ou de marne argileuse, riche en humus.

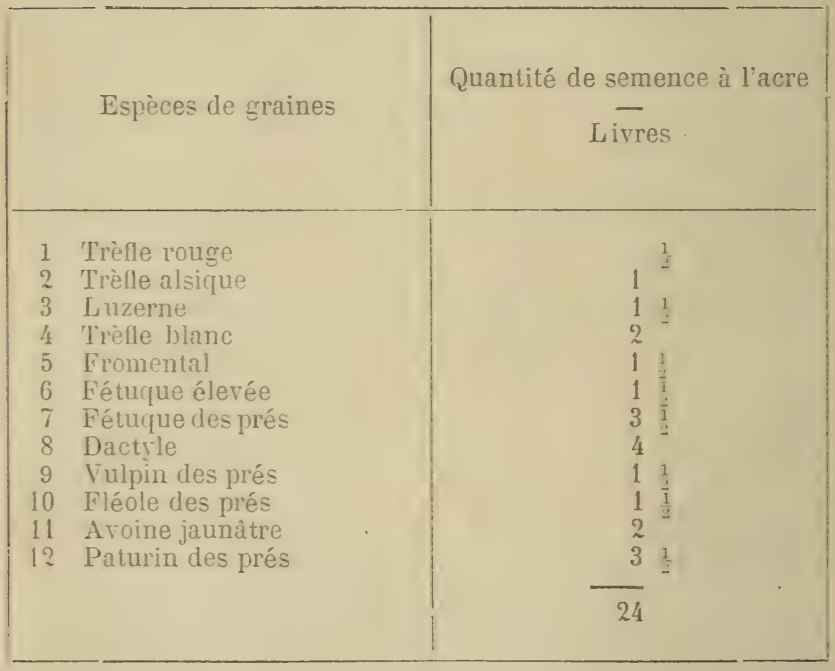

Une boune et fertile terre moyenne ne doit être mise en prairie permanente que quand certaines circonstances la rendent impropre à une exploitation inteusire, telles que son éloiguement de la ferme, la difficulté d'y transporter du fu- 


\section{$-81-$}

mier, etc., etc.-S'il y a profit d'y cultirer un sol intensivement, on fait mieux d'y semer le mélange $A$ pour prairie temporaire.

I.--Mélange pour une terre légère el médiocre

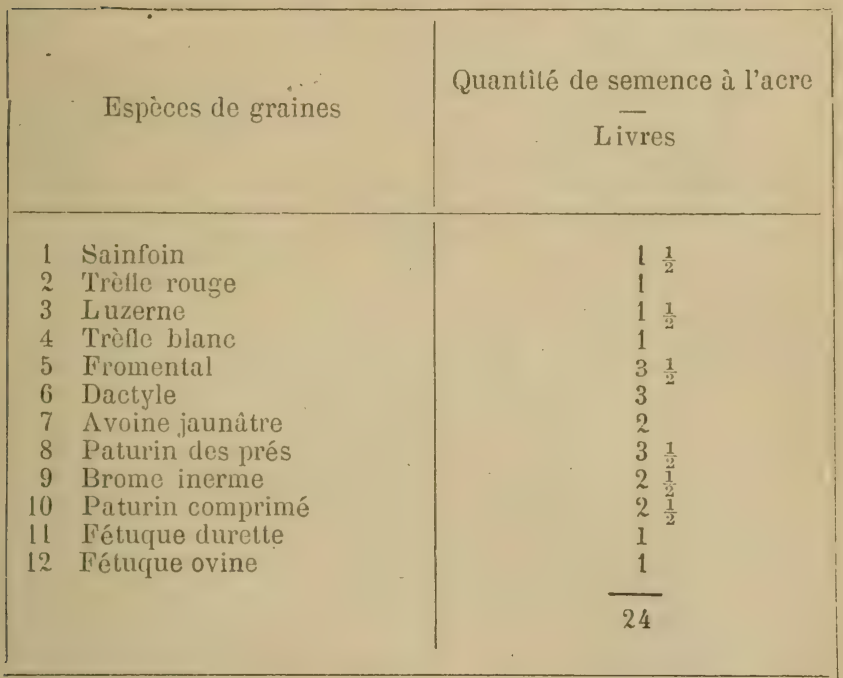

Plus le sol est maigre et léger, plus est difficile le choix de boumes plantes propres à $\mathrm{y}$ réussir. 
J.-Mélange pour une terre argileuse froide.

\begin{tabular}{|c|c|c|}
\hline & Espèces de graines & $\begin{array}{l}\text { Quantité de semence à l'acre } \\
\text { Livres }\end{array}$ \\
\hline \multicolumn{2}{|c|}{$\begin{array}{ll}1 & \text { Trèfle rouge } \\
2 & \text { Trèfle blanc } \\
3 & \text { Trèfle alsique } \\
4 & \text { Fromental } \\
5 & \text { Fétuque des prés } \\
6 & \text { Dactyle } \\
7 & \text { Vulpin des prés } \\
8 & \text { Fléole des prés } \\
9 & \text { Paturin tardif }\end{array}$} & $\begin{array}{ll}1 & \\
1 & \frac{1}{2} \\
2 & \frac{1}{1} \\
1 & \\
2 & \\
3 & \frac{1}{2} \\
5 & \\
3 & \frac{1}{2} \\
3 & \\
23 & \end{array}$ \\
\hline \multicolumn{3}{|c|}{$\begin{array}{c}\text { K.-Mélange pour des prairies irrigures ì sol léger } \\
\text { ou de pesanteur moyenne. }\end{array}$} \\
\hline & Espèces de graines & Quantité de semence ì l'acre \\
\hline $\begin{array}{r}1 \\
2 \\
3 \\
4 \\
5 \\
6 \\
7 \\
8 \\
9 \\
10\end{array}$ & $\begin{array}{l}\text { Trèfle blanc } \\
\text { Trèfle alsique } \\
\text { Fromental } \\
\text { Fétuque élevée } \\
\text { Fétuque des prés } \\
\text { Dactyle } \\
\text { Vulpin des prés } \\
\text { Fléole des prés } \\
\text { Avoine jaunâtre } \\
\text { Paturin des prés }\end{array}$ & $\begin{array}{cc}2 & \frac{1}{2} \\
2 & \frac{1}{2} \\
1 \\
1 \\
4 \\
3 & \frac{1}{3} \\
2 & \frac{1}{2} \\
1 & \frac{1}{2} \\
2 \\
2 & \frac{1}{2} \\
23\end{array}$ \\
\hline
\end{tabular}


L.--Mélange pour une bonne terre tourbouse.

\begin{tabular}{|c|c|c|}
\hline & Espèces de graines & $\begin{array}{l}\text { Quantité de semence à l'acre } \\
\text { Livres }\end{array}$ \\
\hline $\begin{array}{r}1 \\
2 \\
3 \\
4 \\
5 \\
6 \\
7 \\
8 \\
9 \\
10\end{array}$ & $\begin{array}{l}\text { Trèfle. blanc } \\
\text { Trètle alsique } \\
\text { Trèfle rouge } \\
\text { Fromental } \\
\text { Fétuque élevée } \\
\text { Fétuque des prés } \\
\text { Paturin des prés } \\
\text { Dactyle } \\
\text { Vulpin des prés } \\
\text { Fléole des prés }\end{array}$ & \begin{tabular}{ccc}
2 & \\
2 & \\
& $\frac{1}{2}$ \\
1 & $\frac{1}{2}$ \\
1 & $\frac{1}{2}$ \\
4 & \\
1 & $\frac{1}{2}$ \\
4 & $\frac{1}{2}$ \\
2 & $\frac{1}{1}$ \\
2 & $\frac{1}{2}$ \\
\cline { 1 - 1 } 23 &
\end{tabular} \\
\hline
\end{tabular}

Si la terre est forte, froide, diminuez la proportion de fromental et remplacez par la fléole des prés, le dactyle ou le rulpin des prés.

$4^{2}$. Remarques générales sur les mélanges.

1. Les mélanges dont nous renons d'indiquer la composition sont destinés principalement aux prés à faucher. Pour les pâturages, il faut aroir recours surtout aux plantes les plus propres à ce genre d'exploitation, notamment au trefle blanc, 
au paturin des prés et au dactyle pelotonné, qui sont par excellence des espèces à pâturer, tandis que la fléole des prés n'a g'uère de raleur ici. Sur un sol paurre on peut employer aussi la fétuque ovine.

2. Plus le sol est bon, plus il est arantageux de semer dru. Mais il faut y aller prudemment sous peine de subir des préjudices considérables Il importe de ne pas semer à tout hasard sur une moitié d'un acre un mélange calculé pour un acre entier.

3. Pour une terre sèche et un climat sec, il faut aroir recours surtout aux herbes basses, à tallage serré, tel, par exemple, que le trèfle blanc, non seulement en raison de son produit, mais parce qu'il farorise le dépôt de la rosée et la retieut plus longtemps dans ses touffes épaisses, au profit également de la régétation des autres espèces.

4. Les direrses sortes de terraius entre lesquelles nous arons réparti les direrses espèces de plantes fourragères ne comprennent pas précisément tous les mélanges terreux que l'on rencontre. Nous n'a rons indiqué ici que les mélanges principaux, négligeant un certain nombre de sols intermédiaires qui relient ces types entre eux. Lors- 
que l'on aura à ensemencer les terraius intermédiaires, il suffira de choisir les plantes recommandées pour la nature de la terre qui s'en rapproche le plus par sa composition élémentaire et son degré habituel d'humidité. Ainsi, lorsqu'une terre est plus humide que celle indiquée pour tel ou tel mélange $A$ à $M$, le mélange le plus convenable généralement, est celui de la terre qui, par son degré de compacité, se rapprorhe le plus de celle dont il s'agit; ainsi, par exemple, pour une terre de limon humide, le mélange $B$ est préférable au mélange $A$.

5. Il importe de répéter que nos mélanges ne doivent être pris que comme des exemples et non comme des recettes; c'est au cultivateur de composer lui-même les mélanges les mienx appropriés aux conditions dans lesquelles il travaille.

\section{VIII. - Achat des semences.}

Il est rare que le cultivateur recueille sur ses propres prés toutes les semences nécessaires aux mélanges: d'ordinaire, il est obligé de se les proeurer, au moins en partie, par la roie du commerce. En cela, il est indispensable d'user de certaines précautions, afin de ne pas risquer d'avoir une semence mauraise, une récolte manquée ou insuffisante. 
Il est rrai qu'il ne manque pas de marchands honnêtes qui, pourles mélanges aussi, n'emploient que des graines de bonne qualité; mais ceux-là préfèrent que l'acheteur se procure chaque espèce à part, afin de pouroir plus aisément s'assurer de la boune qualité et en estimer la valeur. Il est très difficile de juger d'un mélange de graines, et c'est le cultivateur qui est le moins en mesure de le faire; mais il aura beaucoup moins de peine à apprécier les graines s'il les a sous les yeux en espèces isolées et à l'état de pureté. Pour ces raisons nous conseillons au cultirateur d'acheter à part chaque espèce, pour en composer lui-même ses mélanges, et notamment de tirer sa semence d'une maison de confiance, et qui vend sur garanties. L'acheteur doit exiger la garantie du taux de la pureté et de la faculté germinative. Ayant en mains cette garantie, qu'il fasse contrốler (examiner) la semence achetée, à la Ferme expérimentale centrale d'Ottawa; car il peut arrirer que la garantie soit d'un degré satisfaisant, et que la qualité de la marchandise n'y réponde en aucune façon.

L'examen des grainez, à la Ferme expérimentale centrale d'Ottawa, est fait gratuitement.

Quand un cultivateur n'a pas besoin d'vne grande quantité d'une mềne semence, il peut s'entendre arec son voisin pour en faire l'achat 
en commun. Mais il raudra mieux encore que les cultivateurs d'un même canton, ainsi que les membres des cercles agricoles s'associent pour acheter en commun leur graines.

\section{IX.-Préparation du terrain.}

Du sol, du sous-sol et de la configuration.

Du sol.-Toutes les terres, légères ou fortes ou de nature si différente, que nous avons énumérées en y appropriant les espèces de plantes convenables, peuvent être avantageusement cultivées et converties en prairies temporaires ou permanentes, pourru que ces terres aient assez de profondeur.

Si toutes ont leurs inconvénients, chacume d'elles a aussi ses avantages.

Si l'ou n'a pas le choix, il convient de ne pas regarder de trop près à la nature des terrains. Il y a moyeus de tirer bon parti des uns et des autres.

S'ils sont trop secs et trop légers, il taut leur domner des fumiers humides; les engrais végrétaux enfouis rerts les rafralchissent beaucoup et les affermissent peu à peu. Le grand point, pour ces terres c'est de limontr, - s'il est permis d'employer ce terme,-tous les ans un peu. (Voir l'article Compost). 
S'ils sont trop frais et trop serrés pour laisser passer l'air, il convient de faire des fossés, afin d'en chasser l'eau et d'y amener l'air, ou des rigoles, pour y placer des tuyaux de drainage. On fera, en un mot, au foud de ces terrains ce que l'on fait au fond des pots à fleur, un trou par où l'excès de I'eau s'en ira et par où l'air circulera en formant des nitrates,

L'eau qui dort gâte les racines, le manque d'air les tue. L'eau, comme l'air, doit circuler et se renouveler toujours.

Du sous-sol.-S'il est important de bien se rendre compte du sol sur lequel on doit opérer, il ne l'est pas moins de rérifier arec grand soin la nature du sous-sol. Combien de terrains contenant des éléments de bonne fertilité, sont frappés de stérilité par l'influence mauraise du sous•sol sur lequel ils s'appuient et qui tantôt forme un obstacle à l'écoulement des eaux, tantôt les absorbe arec trop de facilité et derient, en été, tellement sec, qu'il s'échauffe et brûle les raciues des plantes.

Quoique, en règle générale, il faille craindre de ramener à la surface une terre stérile en défonçant ou en labourant profondément certains soussols, il y a cependant lieu, dans les deux cas qui nous occupent, de faire en sorte qu'on arrive à mélanger la couche supérieure avec la couche in. 
férieure, lors même qu'il devrait en résulter une infécondité temporaire.

De la configuration du terrain.

La configuration du terrain doit être prise en grande considération.

Les terrains situés dans les lieux bas, humides, avec des bas-fonds où les eaux ne peuvent s'écouler, sont peu propres aux prairies; il faut de toute nécessité les assainir: "L'eau, qui engrendre l'herbe, engendre aussi le jouc," dit le proverbe allemand.

Les terrains coupés par des éminences à pente rapide, offrent l'inconrénient opposé: l'eau n'y peut séjourner assez longtemps. Le danger est égal, soit qu'il y ait peu d'eau, ou qu'il y en ait trop.

Une pente modérée et égale, une surface unie sans accidents trop prononcés du terrain, qui permette aux eaux de se répandre également et de couler lentement arec régularité, telles sont les conditions les plus farorables pour création d'une bonue prairie.

Du drainage.-Assainissement.

L'opération préliminaire du drainagee, dans les terrains humides, n'est pas moins nécessaire 
pour la création d'une prairie que pour toute autre culture. Un sol marécagenx à l'excès ne conrient pas plus aux régétaux qu'aux animaux et aux hommes.

Le drainage est non seulement nécessaire pour débarrasser le sol de l'eau stagnante, préjudiciable aux herbes qui s'y tronrent, mais aussi pour permettre à l'air d'y pénétrer plus librensent. Il peut, de plus, fournir un supplément précieux d'eau courante.

Plus le sous-sol est dur et serré, plus les drains doirent $\mathrm{y}$ être enfoncés. La profondeur requise pour les drains ordinaires est de 3 a 4 pieds $\frac{1}{4}$ et de quelques pouces de plus pour les drains d'un plus fort diamètre; en général, 3 pieds quand la couche inférienre est sablomneuse.

La distance entre les drains rarie suirant la profondeur des tranchées et la nature du sol; la pente se règle d'après l'inclinaison du terrain à assainir. Les meilleurs drains sont les tuyaux en terre cuite (terre à brique).

Il faut pouroir, en tout temps, s'assurer que les drains fonctionnent bien. L'observation de cette règle est particulièrement intéressante dans un domaine où l'eau peut être employée d'une façon agréable et utile, pour alimenter un bassin, pour l'irrigation, etc. 


\section{$-91-$}

Consert - - Il importe de se rendre compte de la nature du sol arant d'entreprendre cette opération, qui, même sur un espace restreint, pent être honne en certains endroits et inutile ou nuisible dans d'autre : par exemple, si le sol est naturellement sec, léger; ou à un fond sablonneux ou pierreux.

X. Culture préparatoire ou récoltes précédentes, d̀ base de plantes annuelles, sarclíes et amétiorantes.-

Engrais et préparation du sol.

C"est une grande erreur de croire qu'un champ fortement enherbé et encherêtré de racines de chiendent, se prête le mieux, daus cet état, à l'établissement d'une prairie; c'est le contraire qui est rrai. Pour que le mélange puisse réus:ir, il funt que la terre soit exempite de manvaises herbes, bien travaillée et dans un bon état d'engraissement. La récolte précédente qui conrient le mieux est celle qui laisse le sol dans cet état, par exemple les ricolles sarclées, surtout les pommes de terre et les betterares. Car pendant lenr régétation, le sol est biné plusieurs fois, débarrassé des mauraises herbes et bien fumé.

Un champ ainsi cultivé derient libre en automne, et dans l'arière-saison, aiusi que de bonne heure au printemps, il peut être la- 
bouré et fumé, de sorte que la semence est reçue dans une terre meuble, nette et engraissée.

Pour les mèmes raisons, le tabac et principalement le maïs (blé-d'Inde) constituent de très bonnes récoltes préparatoires. Les céréales y sont moins propres parce que d'ordinaire, elles laissent le sol assez ferme, infesté de mauraises herbes et elles ne reçoirent gévéralement pas autant de fumier que les plantes précédentes; nnais, comme le sol en est plus rite débarassé que des plantes-racines, cela permet de lui donner, après la moisson, direrses façons $\in \mathrm{n}$ automne. Quand un semis de graminées doit, au printemps suirant, suivre un blé, il est bon de rompre le champ aussitôt après la moisson. Alors, dès que les mauraises herbesont poussé leurs premières feuilles, on fait passer la herse ; peu de temps après on fume copieusement et en suite on laboure. Si l'on reut faire trois labours en automne, le fumier n'est en force qu'au troisième, et, en ce cas, le deuxième doit être le plus profond.

On peut aussi faire succéder un mélange de graines fourragères à une culture de graminées pures ou de trètles et de graminées, sans que les conditions de la réussite soient beaucoup plus défarorables. Ce procédé est tout indiqué là où le champ ne se prête guère à une autre exploi- 
tation. Dans ce cas, on peut retourner le gazon simplement à la charrue dans le courant du mois d'août, travailler un peu la couche végétale et y ressemer un mélange quelques jours après. Il est avantageux de procéder ainsi ; car, au bout de quatre semaines, on a un nouveau gazon, et l'ancien, qui reste bien en dessous, n'empêche pas les façons ultérieures du sol. Cependant, pour ce dernier, il y a cet incouvénient qu'il ne peut être ameubli et travaillé suffisamment. Alors, il conrient de faire intervenir une demi-jachère, qui s'obtient très bien de la manière suirante: Après la seconde coupe, le gazon est pelé, pour être hersé, fumé et labouré quelques jours après. Dès que le sol a reverdi, on lui donne un ou plusieurs hersages, suivant les besoins et, en automne, un second labour profond, pour être laissé tel quel durant l'hirer. Au printemps, s'il le faut, on laboure et l'on fume encore une fois; et enfin, en mai, on fait un semis. Il est moins laborieux de procéder ainsi : le sol est pelé déjà après la coupe du foin, bien fumé, labouré et ensemencé d'un fourrage rert, dont les meillenrs sont, dans ce cas, le maîs ou les resces. Si la semaille se fait à la fin de juin, on peut déjà couper vers la mi-août. Pendant la végétation de la plante fourragère, le vieux gazon se décompose vite, et, 
l'automne renu, le labourage du sol se fait d'autant plus facilement qu'il a subi une préparation équiralant à celle du mode précédent. Le noureau semis se fait de même en mai. Notons cepeudant qu'à la place d'un fourrage rert, on pourrait aussi mettre des betteraves.

Pour établir une prairie, on commencera donc par fumer et labourer très copieusement le terrain et par le nettoyer au moỵen d'une culture sarclée. binée et rechaussée.

Les arantages physiologiques (fonction des organes) de la culture préparatoire par les plantes sont assez conmues pour qu'il soit inutile de les développer.

On sait que le caractère essentiel de ce système, c'est de faire serrir les plautes elles-mêmes à la prospérité commune, en procurant une bonne récolte de plantes fourragères, tout en améliorant et en nettoyant la terre à convertir en prairie.

Dansce système, la charrue na pas à elle seule, la dure obligation de net toyer et d'ameublir le sol, les cultures sarclées, les plantes étouffantes partagent ce trarail arec les instruments aratoires, et le résultat de toutes ces forces concourant rers un but commun, est une utilisation complète de la faculté productive de la terre. 
ExGrais. Les engrais qui conviement le mieux aux prés, prairies et pâturages, sont les suirants. le fumier des animaux qui rivent de l'herbe, rerte ou séchée, des prairies et pâturag'es, est de beaucoup le meilleur de tous, puisqu'il restitue au sol une bomne partie des substances que les plautes lui enlèrent. Ainsi le fumier de ferme, prorenant de la litière des raches et des cheraux, et le purin de basse-cour, formé par les égouts de ce fumier, sont excellents pour les prés. Les composts, formés de boues de chemius, de curures de fossés ou de mares, de suie, de platras, de sel marin, d'os broyés, de chaux, de superphosphate de chanx, de cendre de bois ou de tourbe, qui produisent des effets merveillenx, le tout arrosé de purin, constituent un des meilleurs engrais pour prés, que l'on puisse employer.

Encrais miruides-Les engrais liquides impriment une grande vigueur à la végétation herbacée.

L'effet du purin sur le gazon est très prompt, pourru qu'il soit appliqué dans de bonnes conditions, c'est-à-dire répavdu en temps de pluie, surtout le soir, à une heure assez arancée pour ériter les roups de soleil. ("est de la sève toule faite, et l'herbe en profite tout de suite. Où l'urine du bétail tombe, sur les pâturages et dans les champs, 
les plantes poussent plus vigoureusement qu'au. tre part; donc l'urine du bétail est un engrais énergique et précieux.

Le purin agit beaucoup plus promptement que les autres engrais,naturels ou artificiels; il agit sur l'herbe en augmentant son abondance et sa vigueur, mais, toutefois, en favorisant peut-être aussi le déreloppement des graminées au détriment des légumineuses, le trèfle blanc excepté.

Ne perdons pas de tue que le phosphate de chaux contribue, dans une forte mesure, à la richesse du lait, que les herbes en enlèvent beaucoup, au sol et qu'il est absolument nécessaire de le lui rendre sous forme de fumure ou de purin. Cette dernière forme est préférable en ce que le phosphate de chaux, étant ell dissolution dans le purin, est tout de suite assimilé par les plantes.

L'acide phosphorique a un effet lent, lors. qu'il est fourni au sol à l'état d'engrais, (engrais minéral, superohosphate du commerce); c'est pourquoi il est préférable de l'appliquer sous forme de purin, car son action n'est pas douteuse sur la qualité de l'herbe des prés, prairies et pâturages.

Comment engraisSer Le sol; FUMURE.-Au début, à l'automne, en préparant le sol, il est plus 
économique et plus aratagenx d'employer les engrais à action lente; c'est pourquoi il est bon d'ajouter au fumier de ferme de la poudre d'os. A u * printemps suirant, à l'ápoque ordinaire des semailles il convient d'appliquer un engrais utilisable un peu plus tôt.

11 sera bon alors de domner une ringtaine de minots de cendres vives de bois et 300 livres de superphosphate de chanx à l'acre. Cependant il ne faut pas mélanger ces deux engrais, mais les épandre séparément, à un intervalle de quelques semaines. A l'automne de l'amnée du semis, on applique en couverture une bonne couche de fumier d'étable bien consommé. Le printemps suivant, on répand sur la jeune herbe un mélange de dix minots de cendres rives de bois et deux cents livres de sulfate d'ammoniaque, à l'acre.

Dans les environs des grandes villes, où l'on peut se procurer facilement dans les grandes usines ou manufactures, de la suie de charbon de terre, une dizaine do minots de suie remplacerait avantageusement le sulfate d'ammoniaque.

Enfin, à l'automme, on donne 25 à 30 tomnes de bon fumier d'étable, et ainsi de suite, d'année en année. A partir de la seconde année du semis, 
quelques arrosages an purin, répétés à direrses reprises, feront merreille.

Il existe encore un autre mode de fumure des prés, que l'on peut, que l'on doit même employer concurremment arec les moyens indiqués ci-dessus, si on rent obtenir des prairies et des pâturages d'un très fort rendement. Il consiste à faire pâturer les prairies et pâturag̣ s a veu soin et à donner en même temps deux lirres de son de blé, et une on deux lirres de tourtuan. moitié de graine de lin et moitié de graine de coton, par jour, à chacun des auimaux qui y paissent.

Il faut aussi toujours plitrer les prairies artificirlles. Cette opération se fait en épandant deux fois, du plâtre sur les press, d'abord, au printemps, lorsque l'herbe a un pouce de longuenr environ ; ensuite, après le pâturag̣e, qui doit géuéralement précéder la récolte de foin (Voir page 185). Le plâtre doit être mélangé arec moitié de cendre. Il convient de n'épandre la première fois que le tiers de la quantité de plâtré (c'est-à-dire environ un minot, la quantité totale à mettre sur macre de prairie étant de trois minots) ; car une plus forte proportion nuirait à la qualité de l'herbr et la rendrait indig̣ste pour le bétail.

La chaux rire en poudre, éteinte à l'abri de l'air, est aussi très arantageuse. Dans ce der- 
nier cas, lorsqu'on emploie la chanx, il faut l'épandre mélangée arec du sable see, si le sol est argileux ou arec de la terre glaise sèche si le terrain est sablomneux, et de la même manière que le plâtre. Il est hou d'alterner ces deux amendements-stimulants : une amnée du plâtre, et l'année suirante de la chaux. La quantité de chanx à épandre sur les prairies est de deux minots à l'acre.

Un épand le plâtre et la chaux, soit à la main, soit au semoir mécanique, le soir, par un temps calme et courert, arant on après tue petite pluie, afin que ces substances sattachent fortement aux fenilles. Il pent être aussi très utile d'ajouter au plâtre et à la chanx, un minot (70 livres) de sel.

La potasse, l'élément principal de la cendre de bois, du muriate de potasse et du chlorure de potassium fait pousser le trèfle. Jules Crerat dit que la richesse en sucre des fourrages est d'autant. plus grande que le sol est plus riche en principes minéraux: potasse, phosphates et calcaire, engrais plus farorables an déreloppement des graines, des racines et des tubercules qu'à celui des feuilles et des tiges ligueuses.

Tne trop forte quantilé de ces engrais minéraux ne convient donc que lorsqu'on rent obtenir de la graine de nos herbages, surtont des légumi- 
nenses. L'azote, qui se troure dans le sulfate d'ammoniaque, et surtout encore dans le bon fumier de ferme, bien conserve. favorise plus particulièrement le déreloppement des graminées.

Ainsi donc, l'engrais par excellence pour les prés, cest le bon fumier de ferme, qui contient beaucoup d'azote.

En ce qui concerne l'engrais, il faut observer que pour le terraiu en prairie temporaire ou perma. nente, il peut se passer beaucoup d'années arant qu'on doire en mettre de nouveau; car les éléments nutritifs des fumures en courerture qu'on lui applique de temps à antre, ainsi que les arrosages au purin qu'ou lui donne, an lieu de pénétrer daus les parties plus profondes, sont absorbés par la partie supérieure de la couche arable. C'est pourqu ui il faut, arant la semaille, engraisser copieusement le sol arec du fumier de ferme.

Les plantes de nos prairies forment, en géné. ral, un gazon épais de plantes graminées pour la plupart à racines superficielles et traçantes ; ces racines tendent toujours à se rapprocher de la surface du sol où elies trourent le mieux leur nourriture dans les feuilles d'arbres, les débris régétaux, les stimulants. les engrais, le limon que leur apportent les eaux. Constamment elles émettent de nourelles radicelles plus superficielles que celles 
qui les ont nourries jusque-là, et qui meurent, contribuant ainsi à augmenter la couche d'humus, richesse accumulée pour l'avenir. "Les herbes des prés, dit M. de Gasparin, ne parviennent à tout leur développement qu'aut aut que parla succession des années, elles se sont formé au-dessus du sol minéral, un terreau azoté pour leurs racines. Quand le gazon n'est pas complètement formé et que les racines des plantes reposent encore sur le sol minéral, si celui-ci n'a pas une richesse naturelle assez élevée, les récoltes des prairies sont encore peu aboudantes, et elles ne parrienment à leur maximum qu'après plusieurs années de régétation et de nombreuses fumures, excepté dans les terrains riches et perméables. Jusqu'à ce point maximum, le fumier distribué aux prairies ne produit pas tous ses effets, et ce n'est que quand elles y sout parvenues qu'on peut espérer de voir reproduire sa véritable valeur." (Cours d'agrimllure, t. I., p. 690.)

Pour un semis de printemps, le mienx est d'enterrer le fumier en automue par un labour, parce que, comme il se décompose un peu pendant l'hiver, les jemnes plantes troureront plus sûrement de la nourriture déjà prête en quantité suffisante. Si l'on ne peut fumer en automne, il faudra le faire de bonne heure au printemps, afin que la terre puisse en profiter arant l'ensemencement. 


\section{$-102-$}

Daus les lieux où le sous-sol et la couche arable sont de même composition, une culture profonde peut aussi rendre d'excellents serrices pour les mélanges de plantes fourragères; celles-ci étant alors à même de plonger plus bas arec leurs racines, rencontrent de nouveaux éléments uutritifs qui ne leur étaient pas accessibles aupararant. Tje cette manière, elles ont aussi moins à souffrir de la sécheresse, ce qui est arantageux surtout aux grraminées. De plus, l'excès d'humidité se répartissant aussi mieux dans une terre ameublie, les plantes y sont moins éprourées dans les années très humides. Les agents atmosphériques, air, chaleur, eau, ont plus daction sur me terre ainsi préparée et dissolrent des éléments minéraux res. tés insolubles jusque-là. C'est pourquoi le rapport des cultures fourragères est plus considérable arec un sous-sol ameubii. Un labour profond est pì arantageux s'il est fait en automne, car en hirer le sol est désagrégé par les alternatires des grelées et des dégels. Quand une quantité trop forte d'un sous-sol non traraillé jusqu'alors est amenée tout d'un coup à la surface et mêlée à la couche végétale, la conséquence n'en peut être que nuisible. C'est pourquoi il ne faut procéder que peu à peu à l'approfondissement de cette couche. Pour commencer, il raut mieux se boruer plutôt à 
ameublir le sous-sol, sans le porter à la surface, au moyen d'une charrue fouilleuse. Quand on n'a pas de charrue fouilleuse, on peut arriver au même résuitat par l'emploi successif de deux charrues: l'nne avec son versoir, et l'autre sans versoir ni avant-train, mais arec le fer incliné de 8 à 16 pouces à droite. On fait un tour entier avec la charrve complète, puis on refait ce même tour, en plongeant l'autre charrue daus la rai :, tracée d'abord; et on continue ainsi pour chaque tour. Le sous-sol se trouve alors suffisamment ameubii, saus qu'on ait eu à craindre de nuire au sol.

Cette opération se nomme le défoncement On emploie ensuite de nonrear la charrue ordinaire. On continue ce mode d'amélioration du sol, labourant de plus en plus profondément, jusqu'à ce qu'on ait atteint le fond de la couche ameublie du sous-sol.

Les façons culturales, en vue d'un semis de graines fourragères, doivent se faire en antomne, si possible: car, après l'hirer, la terre est bien divisée, et il suffit d'y faire un 'éger labour. suivi de hersages énergiques, pour qu'elle soit prête à l'ensemencement. Si l'on ne pent procéder ainsi, il faut émietter le sol par des traraux répétés arec la charrue, la herse, le scarificateur, etc.; car on ne peut attendre de hons résultats de l'ensemence- 
ment d'un champ mal préparé et courert de mottes plus ou moins grosses.

Dans un tel terrain, les graines plus fines tombent à une profondẹur trop grande et s'y perdent inéritablement, tandis que celles qui restent sur les mottes ne lèrent qu'en partie et périssent plus tard.

Un semis de graines fourragères exige donc que le sol soit préparé presque aussi bien que la terre diun jardin potager.

LES COMPOSTS, LES BALAYURES,LES BOUES ET LES IAIMONS.

Presque toutes les herbes aiment la fra1cheur, non seulement aux racines, mais aussi aux feuilles; il s'ensuit que notre climat ne satisfait exactement pas à ces conditions. Voilà la première et la plus grande difficulté, contre laquelle on luttera sourent en rain.

Pour en qui est du sol, plus il sera léger, moins il conriendra; au contraire, s'il est argileux les herbes y réussiront.

Tandis que par conséquent sur une côte aride et sablonneuse, il est presque impossible d'obtenir une belle prairı, une rallée basse et argileuse est très propice. Cela ne suffit pas cependant; il faut encore bien préparer le sol et soigner les herbes. 
Voici, d'après M. Van Hulle, ce qu'il y a de mieux à faire, indépendamment de ce qui a été recommandé ci-dessus, nou seulement pour créer un beau pré dans les situations farorables, mais encore pour lui assurer de la durée, même dans de très mauvaises conditions :

"Ne pourrait-on pas attribuer, en très grrande partie, la beauté du gazon le long de la voie publique à la poussière que le vent y chasse et qui recouvre et abrite constamment les racines supérieures, les plus importantes, au fur et à mesure, pour ainsi dire, qu'elles se forment, tandis que, dans nos prairies et pâturages, ces racines supérieures sont rôties par le soleil? Dans l'affirmative,--et nous croyons que cela n'est pas douteux.-il suffirait d'imiter plus ou moins ce qui se passe dans la nature, c'est-à-dire de répandre de temps à autre une lég’ère couche de terre sur le pré. Il ra de soi que, plus cette terre sera fertile ou substantielle mieux cela vaudra; toutefois, au besoin, durant l'été surtout, toute terre, quelque légère qu'elle soit, doit produire un excellent effet. Tâchous donc toujours d'aroir quelque part en dépôt un tas de terre, et chaque fois que, par ci par là, une place laisse à désirer, répandons-y environ quatre lignes (un demi pouce), de cette terre, durant tout l'été." 
"Le temps le plus propice pour transnorter les boues sur les prairies, est l'hirer; en roici les raisons: la première, c'est que par là on érite de briser le sol des prés arec les roues des roitures d'été, qui fouleraient trop violemment et trop inégalement le terrain ; la seconde, et c'est la principale, c'est que l'hirer est le meilleur pulvérisateur, et que, le printemps arriré, il laisse un engrais-amendement admirablement préparé pour être répandu sur les prés, prairies et pâturagees. De fait, c'est au printemps, de bonne heure, arant la reprise de la régétation, qu'il convient de limoner les prairies."

\section{RESTITUTIUN AU SOL DE SA FERTILITÉ}

Nous ne saurions mieux faire que de reproduire, à ce sujet, les paroles de M. Fouquet, membre du Conseil Supérieur d'Agriculture et professeur à l'Institut Agricole de Gembloux, Belgique, appelant, au congrès national d'agriculture, l'attention de l'assemblée sur les soins à donner aux prairies:

"Elles sont généralement mal soignées, dit-il, surtout au point de rue des engrais. On les considère trop sourent comme des magasins fourragers inépuisables. On croit que les prairies n'ont pas besoin de fumures, ce qui est une erreur : les plan. 
tes des prairies ont les mêmes exigeneas que celles de nos cultures aunuelles, d'autant plus même qu'elles occupent le sol d'une façon permaneute.

"Le rendement moyen des prairies est peu élevé, et c'est à l'aug'menter surtout que le cultivateur doit chercher à arrirer; l'augmentation de produits équivaut, en effet, à une extension en surface et permet de nourrir un plus grand nombre de têtes de bétail sur une exploitation donnée."

M. Fouquet rappelle que des expériences faites à Rothamsted (Angleterre), sont concluantes et que, par des fumures conrenablement fournies aux prainies, le rendement de celles-ci doit augmenter. Il proure que le fumier de ferme fabriqué avec les seules ressources de l'exploitation, n'est pas suffisant pour maintenir la fertilité des terres arables et, des prairies tout à la fois, et il indique des sources auxquelles le cultirateur peut aroir recours pour suppléer au manque d'engrais de l'exploitation.

Voici quelques chiffres qu'il a empruntés aux nombreux travaux de Lawes et Gilbert, à liothamsted. Ces chiffres se rapportent à l'acre, 
FOIN

Bottes.

Sans engrais.

Sulfat: ef chlorhy rrate d'ammoniargu.

Nitrate dr sourte..........................

Superphosphate de chaux .......................

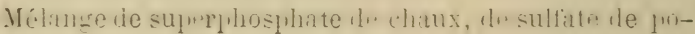
Lasse, de soude et de magnésie..................

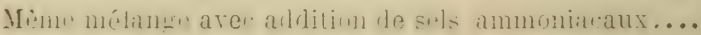
Mime mélanwn, linitrate de sulule minplarant les sels ammin

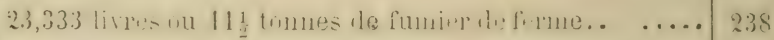

23.333 livms an 11 1 trunts de fumier le forme et sels ammoniacaux

....

$140 \frac{1}{2}$

$1851 / 2$

$1891 / 2$

$1571 / 2$

1951/2

310

285

$2721 / 2$

"C'es chiffres, dit M. Fouquet, ne laissent aucun doute sur les arantages qu'il peut y aroir à traiter les terrains enherbés arec moins de parcimonie que nombre de cultivateurs n'ont l'habitude le faire."

Les graminées, qui constituent la base des plantes de prairies, ont les mêmes besoins que les céréales; elles sont cultivées, en geénéral, pour leurs tiges, lesquelles doivent être consommées sur pied ou fauchées au début de la floraison, qui 
coincide arec le maximum de valeur alimentaire de la récolte. Le cultirateur ne peut done pas per. dre de rue que la teneur élevée de la récolte en acide phosphorique, en chaux et en potasse, est une preure certaine de leur valeur nutritive éle. rée et que, au contraire, là où les minéraux ont été fournis et absorbés par la récolte en quantité insuffisante, la valeur alimentaire est faible.

Daus beaucoup de cultures, dit le Guide du Cultivateur. de France, on adopte un engrais incomplet dosant eaviron : azote, 6 pour cent, acide phosphorique soluble et assimilable, 6 pour cent.

En revanche, l'addition de potasse produit habituellement un effet très marqué dans tous les terrains légers et pauvres en minéraux.

La dose de potasse ajoutée varie alors de 2 à 7 pour cent.

Voici la formule de M. Georges Ville pour l'engrais complet :

\begin{tabular}{|c|c|}
\hline 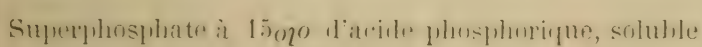 & Livres \\
\hline 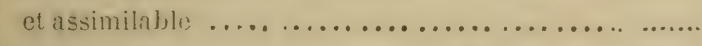 & $\therefore: 10$ \\
\hline Nitrate de potasse. .......... & .75 \\
\hline Sulfate d'ammoniaque.......... & 50 \\
\hline Sulfate de chaux.... & 150 \\
\hline Total......... & 425 \\
\hline
\end{tabular}


Ces chiffres s'adaptent à la quantité d'engrais nécessaires pour un acre de terre et épandus en courerture sur les prairies déjà établies.

"Cette proportion de potasse est considérable, ajoute le Guide du Cultivateur, et n'est justifiée que là où le sol en est très appaurri par une culture épuisante, sans restitution suffisante ou sil en contjent naturellement très peu, ce qui est surtout le cas d'un grand nombre de sables."

\section{SAISON PROPIC'E A LA RESTITUTION AU SOL.}

Il faut, autint que posible, appliquer ces amendements-engrais arant l'hirer. Épandus en octobre, ces engrais derienn:nt plus facilement assimilables pour que la première compe en protite largenent, dès que l'herbe se réveille en arril-mai ; tandis que, einployés seulement au printemps, ils ne produiraient leur effet que plus tard.

Cependant, les sels ammoniacaux, et les nitrates surtout, de même que les engrais liquides, ne doirent être épandus qu'au commencement du printemps. Le sulfate de chaux, ou plâtre, ne doit même être emplosé que lorsqu'il n'y a plus aucune gelée à craindre. 


\section{$-111-$}

Nous croyons deroir domner ci-après un exemple de préparation et de fumure d'un terrain devant servir de prairie, arec du fumier de ferme et des engrais chimiques combinés.D'abord, la première chose à considérer pour la création d'un pré (prairie et, pâturage), est l'espèce de terre qui convient le mieux. La compacité du sol est une condition rigoureuse d'une forte production herbacée. On choisira donc une terre assez fortement aroileuse, pas à l'excès cependant, mais très bien drainée, ou ayec un bon sous-sol perméa. ble.

En l'absence de l'une de ces deux conditions, il faut pratiquer le défong̨age, opération qui consiste à remuer le sol arec une harrue fouilleuse ou par l'emploi successif de deux charrues ordmaires. De cette manière le sous sol se trourera suffisamment ameubli et mettra à même d'assécher assez bien le terrain, en supposant toutelois que les drains fonc. tionnent bien et que les canaux et les rigoles soient dans un bon état. De fait, les meilleures espèces d'herbes ne croissent jamais dans les endroits humides. Tous les animaux de ferme profiteut plus rite dans les terres sèches (parfaitement égouttées, assainies), ils s'y couchent plus à leur aise, et la saveur de l'herbe y est plus appétissante; par suite les produits sont supérieurs. Il fant choisir uue 
terre forte et riche, car il faut que le terrain soit saluré des éléments fertilisants des plantes qu'on a l'intention d'y cultiver. Il faut de plus que laculture des plantes-racines, qui doit toujours précéder l'établissement d'uu pré, ait mis la terre dans un bon état de culture. Si l'on veut obtenir des rendements extraordinaires, il faut que la terre ait été rendue aussi meuble que celle d un jardin. Les rarines de toutes les espèces d'herbes de prairies et de pâturages sont excessirement fines, et ne peurent pénétrer aisément les mottes de terre.

Il faut cornmencer à préparer la terre pour l'établissement d'un pré, l'automne qui précède l'année du semis, en labourant et défonçant le terrain, après aroir épandu sur le champ une bonne quantité de chaux rive en poudre (40 minots à l'acre, en moyeune). Si la terre est très forte, on fait un noureau labour sur la largeur du champ, le premier ayant été fait sur la longueur, ce second labour devra être le plus profond; enfin, on en fait un troisième, mais léger, (environ deux ou trois semaines après le premier), et on y enterre 25 à 30 tonues de fumier pailleux. Car ici il ne sagit pas seulement d'engraisser la terre, il faut de plus l'umenler: la rendre meuble aussi profondément que possible, si ce travail n’a pas cléjà été fait d'une manière satisfaisante pour les plantes-racines (pa- 


\section{$-113-$}

tates, navets, etc.) On ajoute au fumier d'étable 300 livres de poudre d'os, à l'acre.

La saison la plus propice pour les labours est l'automne, parce que nos fortes gelées ont pour effet de désagréger le sol. De fait, l'hiver est le meilleur des scarificateurs.

Au printemps suivant, noureaux labours sur les deux sens, à la profondeur du dernier labour fait l'automne précédent, qui a servi à enterrer l'engrais. Ensuite on herse énergiquement en tous sens avec la herse-bêche, la herse à disque, la herse ordinaire, et on roule fortement arec le rouleau brise-mottes et le rouleau ordinaire; puis on laisse réchauffer la terre au soleil pendant quelques jours.

XI. - Epoque DEs SEMAILles. - DIVERses maNIÈRES DE SEMER. - SEMAILLES DES GRAINES FOURRAGì̀RES SANS CÉRÉALES.

La semaille des graines fourragères demande de l'attention Le temps le plus favorable pour les semailles des mélanges est la fin de l'été (mois d'août.) En voici les raisons. D'abord les graines reuant de mûrir sont plus frafches et ont par suite une faculté germinative plus grande; ensuite, les semis de fin d'élé, étant farorisés par l'humidité et la boune température de a saison! 
lèveront mieux que ceux faits au printemps, où l'on a toujours à redouter les temps secs, chauds et venteux, si desséchants et si désastreux, qui font sourent que les semis de printemps échouentcomplètement. La nature sème toujjours à la fin de l'été, suirons ses indications et les jeunes plantes seront déjà assez fortes, assez robustes, assez bien emracinées et installées à l'automne, pour se bien défendre contre les froids de l'hirer; ct la prairie sera établie au retour de la belle saison. Dès le mois de juin, elle pourra fouruir un très bon pâturage pour raches laitières, ou, si l'on attend le mois suirant, une binne coupe de foin; tandis que, d'un sernis de printemps, on ne peut espérer qu'une petite herbe qu'il est préférable de ne pas faire pâturer du tout arant les neiges. Par le semis de fin d'été, on peut donc, comme on le roit, gagner presque une année.

Il y a deux modes de semer les mélanges, arec ou sans semence de céréales. Selon nous, la dernière manière est de beauconp préférable, pour la raison suirante: chaque graine aura la chance de profiter de la nourriture qui lui convient, sans crainte que les racines des grains l'eu privent. La nature sème toujour's les graines d'herbes sans mélange de grains. Imitons-la done, elle comnaît bien son métier. 
Si l'on emploie le deuxième mode de semailles, celui de confier les graines ì la terre sans additions de céréales, on doit semer dans le courant du mois d'août.

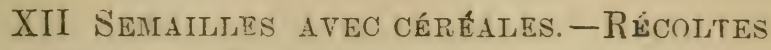
PROTECTRICES.-ENTERREMENT DE LA SEMENCE.

A ceux qui ne voudraient pas risquer de semer les graines de mélanges sans orge, hlé, ou avoine, par la crainte que l'herbe ne manque et ne fasse perdre ainsi tout le profit de l'année, bien qu'il soit certain que l'herbe pousserait plus vite et deviendrait plus touffue étant semée seule, à ceux-là nous recommanderons de faire un léger ensemencement d'aroine, qui sera fauchée en vert. Dans ce cas les semailles doirent se faire au printemps.

L'avoine en vert est bien en effet la céréale protectrice la plus propre et la plus sûre, tant pour les graminées que pour les léguminuses. Elle donne un bon abri aux jeunes plantes four. ragères, sans occuper la place longtemps, et fournit elle-même, à l'époque où celles-là ne sont pas encore développées, un bon rendement de fourrage. - L'avoine se sème dans la proportion d'un minot environ à l'acre. Mise au printemps, elle acquiert en quatre ou six semaines, une hauteur d'environ six pouces, et doit alors, dans 
tne journée chaude, être coupée un pent haut, afin que les jeunes plantes fourragères, reçoirent de la lumière. Si on laisse l'aroine s'élever beaucoup plus, la réussite d'un semis si dru est compromise, tandis que, en fauchant de bonne heure, non seulement toutes ces plantes prenuent un meilleur aspect, mais aussi le rapport en aroine rerte est plus considérable, parce qu'a?ors l'aroine fournit deux coupes. Après cela l'aroine repousse dru et rend considérablement. On peut laisser cette dernière grandir plus que la première fois, mais sans toutefois trop attendre pour la seconde coupe, de crainte qu'elle n'affaiblisse trop les jeunes plantes fourragères. Comme la première. la seconde coupe doit aussi être pratiquée haut, afin de favoriser le tallage des graminées. Après cette seconde opération, l'aroine reste en arrière, et les graminées et les légumineuses dominent, s'enracinent fortement, forment un bou gazon, et pourraient même fournir une troisième coupe de fourrage; mais il raut mieux les laisser sur pied pour protéger les racines des jeunes plantes contre les froids de l'hiver.

Si la première coupe se fait trop tard. ce n'est pas seulement le mélange qui en souffre, mais l'aroine derient trop maigre à la seconde compe 


\section{$-117-$}

et rend moins que par le procédé que nous recommandons.

Quand on fait simultanément la semaille d'un mélange et de la récolte protectrice, il faut commencer par mettre et enterrer la semence de celle-ci, parce que toutes les graines ne reulent pas être enfouies à une égale profondeur. Une graine minuscule, qui est de cent à deux cents fois plus menue qu'un grain d'aroine, ne doit pas ètre enterrée aussi profondément que celui-ci.

Si la récolte protectrice est une céréale prour grains, il est à recommander de semer en lignes, parce qu'alors les plantes fourragères auront moins à souffrir de l'ombrage. Si l'on sème à la rolée, la récolte protectrice doit être hersée d'abord. Si ou met du sainfoin (esparcette) dans le mélange, on peut le semer et le herser arec la récolte pro. tectrice, parce qu'il exige d'être couvert autant que la céréale. Ce n'rst qu'après l'enterrement de ces deux semences qu'on peut semer les trèfles et les graminées, car leurs graines périraient pour la plupart si elles étaient enfoncées aussi profondément que la céréale: il n'y a que les graminées à grosses graines, comme le fromental, qui germeraient en partie. I)ans les terres fortes, le semis des trèfles et des graminées ne reçoit qu'un roulage; mais dans une terre légère, tous les semis 
do ivent être hersés, sauf ceux du paturin des prés fu'il faut toujours se borner à rouler.

De ce qui précède, il ressort déjà que les dirèrses espèces d'un mélange ne doivent pas être semées ensemble, et cela non seulement à cause de l'enterrement des semences mais aussi par suite des différentes natures de sol. Les graines lourdes, comme celles des trèfles et de la fléole des prés ne se laissent pas bien mêler arec les légumineuses; elles descendent au fond du mélange et, semées à la rolée, sont lancées bien plus loin que le fromental ou les autres graines légères, garnies de glumelles et de poils. C"est pourquoi les graines lourdes et les légères ne doirent pas être semées ensemble, même quand il les faut enterrer à la même profondeur : la semaille des unes et des autres doit se faire séparément.

Ce qui précède doit être mis ell pratique aussi bien pour les semailles de mélanges sans céréales que pour celles avec grain.

Voici maintenant un aperęu de la manière de procéder :

A.- EN TERRE Forte

1. D'abord, semaille et hersage de la récolte 
protectrice (avoine, etc.) et de l'esparcette (sainfoin).

2. Ensuite mélange et semaille des graines lourdes.

\section{Portion $I$}

(a). Toutes les espèces de trèfles et d'autres légumineuses ;

(b). La fléole des prés;

(c). Le dactyle, en graines sans glumelles (enveloppes) ;

(d). Le paturin des prés, si la graine a été battue, nettoyée, et si elle est à peu près pure.

3. Toutes ces graines étant semées, on passe immédiatement au mélange et à la semaille de la portion II qui comprend:

\section{Portion II.}

(a). Le fromental ;

(b). La fétuque des prés;

(c). Le dactyle, en graines à glumelles;

(d). Le vulpin des prés;

(e). Les fétuques fines;

$(f)$. L'avoine jaunâtre;

$(g)$. Le paturin, à graine lég’ère ;

Après quoi, les deux portious (I et II) subissent un roulage commun. 


\section{B.-EN TERRE LÉĠ̀re.}

1. Cornme ci-dessus, d'abord semaille de la récolte protectrice; et si l'esparcette (sainfoin) doit entrer dans le mélange, elle peut être semée et hersée en même temps

2. Ensuite, semaille de toutes les espèces de légumineuses et de graminées, sauf le paturin, (portion III)-bien entendu en mettant séparément la semence lourde (portion I) et la légère (portion II)-arec hersage extrêmement léger.

3. Enfin, semaille de la graminée réservée (portion III), et roulage du champ.-Il est clair que l'ensemble de ces opérations n'a lieu que lorsque le mélange comprend toutes les graines en question.

Afin que la semence soit répartie également, il importe de faire de chaque portion un ensemencement croisé, qui consiste à en répandre une moitié daus le sens de la longueur du champ et l'autre moitié dans le sens de la largeur ou en travers; car rien n'est plus ennuyeux que de roir plus tard des lacunes apparaitre dans le champ. Mais ce qui est surtout très fâcheux, c'est que le rapport en est diminué et que ces lacunes sont difficiles et coûteuses à combler. C'est pourquoi il ne faut pas craindre le surcroît de travail exigé 
par l'ensemensement croisé, car on le regaggue largement dans une exploitation qui dure plusieurs années.

Il y a un excellent instrurnent, le semoir d trèfle qui ne derrait manquer dans aucun graud domaine et dont l'acquisition est à recommander aux sociétés agricoles.

Arec son aide, un homme pent ensemencer dans un jour dix-huit acres, et la semence étant ainsi répandue plus également qu'à la main semant à la volée, il résulte non seulement une économie de graine, mais encore un engazonnement plus uniforme et un rendement plus considérable. Le semoir à bras ne peut naturellement être employé que pour les petites surfaces; pour les grandes exploitations on se sert de semoirs à cheval.

Bien que ces instruments soient construits spécralement pour la semaille des trèfles, ils peurent aussi, sans rien y changer, servir pour les graines de graminées, à l'unique exception de celles du fromental. Mais il faut aroir soin d'ouvrir entièrement les orifices. Il est bon de semer séparément les graminées et les trèfles, auxquels on peut joindre la fléole des prés. Toutefois, ce n'est pas absolument nécessaire, 
surtout si la caisse ne se remplit pas complètement de semence et si on ne fait qu'en ajouter aux deux bouts du champ. - Il existe un semoir à trèfle à orifices orales et plus grands, arec lequel on peut semer toutes les graminées, y compris le fromental.

XIII-SOINS D'ENTRETIEN DES PRAIRIES.

Si l'on reut qu'une prairie présente un beau gazon bien fourni et qu'elle rapporte le plus possible, il faut qu'uprès la semaille elle soit l'objet de soins contenables, et nous allons maintenant donner là-dessus quelqu s conseils.

Pendant la première amníe, la prairie ne derrait pas être arrosée de puriu, à moins que cet pugrais liquide ne soit bien fermenté et fort étendu d'eau, saus quoi il serait préjudiciable aux jeunes plantes, notamment aux graminées fines. Au lieu de rela, ii raut mieux courrir le champ de fumier pailleux par lequel. non seulement il sera fertilisé, mais aura l'arantage d’ètre protégé pendant l'hiver. Il ne conrient pas non plus d'y amener du compost, la première année, si ce n'est quand il y a des lacunes auxquelles on reuille remédier le printemps suirant par de petits semis supplémentaires

Il importe de soumettre le sol à un roulage 
avec un instrument des plus pesants, non seule. ment après la semaille mais encore après la pre. mière coupe et en automne; mais cela est surtout très utile au printemps suivant, quand la terre est devenue sèche, car alors il se trouve souvent que les plantes ont été en grande partie déchaussées pendant l'hirer et montrent leurs racines dénudées à moitié ou entièrement; or, colles-ci doirent être enterrées de noureau, sinon, une partie des plantes qu'elles sont chargées de nourrir dépériront on resteront ficibles. Beaucoup de graminées telle que la fétuque durette, etc., ont la propriété de se former un pied en toulle épaisse et d'une certaine hauteur, et de produire par là des inégalités dans la surface enherbée. A u moyen du rouleau, ces saillies sont aplanies au niveau du reste, et le gazon de la prairie redevient uniforme et plus commode à faucher. L'emploi du rouleau sur les prairies et les pâturages, est donc fort avantageux, quoique rarement pratiqué. Il a nou seulemunt pour but et pour résultat de refouler le gazon souleré par les golées, mais encore celui de chasser devant lui l'excès d'eau qui peut se reucoutrer sous ce gazon. Lus prés consolidés et ressuyés par ce moyen, à la sortic de l'hiver, régètent plus tôt, plus vigoureusement, l'herbe se garnit mieux du pied et croit plus 
tard à l'automne, que si l'on négligeait le roulage. Ce roulage ilevrait étre répété pendant les premières unnées, et méme il est avantageux de le faire chaque printemps deux ou trois fois, ainsi que chaque automne.

Pour les prairies permanentes, notamment celles qui sont de rieilles prairies naturelles, il est sourent tres arantageux de donner un hersage superficiel arec la herse à pré. Quand le sol est recourert d'un feutrage formé par les tiges radicantes, soit du paturin commun, soit de l'agrostide commune ou trainesse. les graminées autres et plus productires en souffrent considérablement. Elles finissent par être étouffées dans les mailles de ce tissu serré, qui ne laisse qu'un accès insuffisant à l'air et à la chaleur. A cet inconrénient se joint sourent celui d'un développement de mousse, d'acides régétaux nuisibles, qui, à leur tour, farorisent la pousse de mauraises herbes acides. C'est ainsi que nous royons souvent les meilleurs terrains punir la négligence du cultivateur en se recouvrant de mousses et de laiches, derant lesquelles les bonnes graminées ont disparu peu à peu. Le produit, comme quantité et comme qualité, n'est plus que très médiocre. ("est alor's qu'il s'agit d'aroir recours aux bons offices de la herse à pré. Après qu'elle a déchiré la couche de 
mousse et d'herbes feutrées, la terre est ourerte de nouveau à l'influence de l'air et de la chaleur et, en peu de temps, il apparaît une toute autre végétation : les graminées et les légumineuses reviennent en bonnes et productires espèces, et le rendement de fourrage ra s'accroissant de plus en plus.

"La herse, dit Joigneaux, détruit la mousse s'il y en a, coupe un certain nombre de racines, de façon à en faire repousser quantité de petites; la herse ourre les raies qui domnent de l'air au gazon en dessus et en dessous, qui permettent à l'eau chargée d'engrais de pénétrer parfaitement dans le sol et de produire une végétation vigoureuse."

F. Andcreg'a a fait les expériences suivantes, qui attestent de la manière la plus frappante la grande utilité du hersage.

lime parenle: ni hersén ni lumére, ralpurt: 151 holles de linin. 2e " fumée et non horsée

3e " non fuméc, mais hersée

40 " herséc ęt fumée

111 " "

$102 \frac{2}{3}$ "

$208 \frac{1}{3}$ "

C'est surtout daus les prairies vieilles et infestées de mousse et d'herbes fentrées, que le produit peut être relevé considérablement par le moyen du hersage. L'acquisition d'une herse ì pré est donc fort à recommander aux cultivateurs. 
Il serait bon surtout, de la part des petites sociétés locales (cercles agricoles), de s'en procurer une, pour mettre à la disposition de leurs membres ; ou bien plusieurs enltivateurs pourraient s'associer pour l'acheter et l'emplojer tour à tour. l'un d'eux étant chargé de la garde de l'instrument.-Jusqu'ici l'un a fait nsage principalement de deux sortes de herses à pré: l'ume à dents an fonte, faisant corps areo les membres, et l'autre à dents cn acier rissées dans les membres et pourant se remplacer quand elles sont usées. C'ette dernière est préférable.

Un instrument très recommandable et bien plus arantageux que le précédent, est la lierie a ressart. Les membres ne sont pas sujets à se casser et les dents sont par paires; celles que le trarail a émoussées, peurent être changées facilo. ment par le premier ouvrier venu.

La herse a encore pour but l'extirpation dautres mauraises herbes, notamment de celles qui s'étendent en rampant sur le sol, telles que la renoncule rampante, les réroniçues printanière et à feuilles de lierre, la bugle rampante, lo lierre terrestre, etc. Elles serrent aussi à nireler les déjections des rers de terre. les taupinières et les fourmilières ainsi qu'à diriser le compost ou le fumier apporté sur le champ, et ce sont des 
instruments très propres à cet usage. Le hersage se lait au printemps, à la fin d'arril. Les parcelles de mousse arrachée et les mauvaises herbes sont ramassées pour la fosse à compost. Après que la prairie a été hersée, on peut encore y semer un mélange de graines convenables, d'entiron $\frac{1}{3}$ is de la quantité ordinaire, suirant que le gazon est déjà plus ou moins dru; c'epundant il n: faudrai pas trop espérer de ces semis supplémentaires, quoique dans certaines circonstances farorables, on les ait vus soavent domner de très bons résultats. En tout cas, il faut faire suivre le hersage d'un énergique roulage, mème quand on ue domne point un semis supplénentaire, parce que les bonnes plantes qui ont été inises à décourert par la herse doirent de noureau être pressées dans le sol.

Quant aux mauraises herbes, dont la souche ou les bulbes sont à me assez grande profondeur dans la terre, elles ne se laissent pas, il est rrai, extirper par la herse à pré, et il faut recourir à d'autres procédés.

Pour la destrution du colchique d'aulomne. ¿Colchicum aztumnale', l'on nous a déjà recommandé une multitude de noyens, mais nous n'en connaissous ancun arec lequel on puisse, sans trop de peine, se rendre maitre de cette plante si 
détestée des cultivateurs. Le plus sûr encore c'est d'améliorer l'état physique du sol par le drainage et, si possible, par un labour suiri d'un noureau semis. Il en est de méme de la RenonCULE ACRE (Ranunculus acris,).

Les grandes espèces de Patiences (Rumex obtusifolius, R. Pulientin, s'extiripent le mieux arec le levier à deux dents.

Le Persil D'ane (Authilicus sylvestris) et la Berce Brancursine (Flerechim sphondylium) aboudent dans le roisinage des fermes et daus les rergers, et cela rient principalement d'un usage exclusif du purin comme engrais ou de l'emploi de ce liquide à l'élat non fermenté ou par trop concentré. Si, en place de purin, l'on se sert pendant quelques anmées de poudre d'os sulfaté, (superphosphate de chaux), ces mauraises herbes disparaissent peu à peu. Mais le procédé d'extirpation le plus simple serait de labourer le sol, d'enlerer soigneusement les fragments de racines et de semer à noureau.

Les prairies irrigables (susceptibles d'être arrosées) nourellement ensemencées, ne doirent être arrosées que très peu pendant les deux premières années. 
DESTRUCTION DES MOUSSES DANS LES PRAIRIES.

Des essais faits en Angleterre par M. Edg'son, dit M. l'ingénieur P. Marguerite-Delasharbonny, dans le Journal de l'Agriculture, auquel nous empruntons ces renseignements, ont montré que le sulfate de fer (couperose verte) répandu sur les prairies, les débarrassait complètement de ce pa. rasite. Cette destruction, suirant M. Griffiths, était due à la grande quantité d'oxyde de fer absorbée par les mousses, leur cendre en contenant plus de 10 pour cent, dose estimée, par cet expérimentateur, mortelle pour les régétaux.

Aussitôt que M. Gustare Michiels eut connaissance de ces résultats, il institua des expériences pour les contrôler: il répandit en mars, sur une partie infestée par les mousses, du sulfate de fer à la dose de 170 livres à l'acre. Un mois après elles avaient disparu complètement, sans que l'herbe eût souffert de la présence du sulfate de fer.

Depuis cette époque, les mousses ayant repoussé en quelques points, il leur appliqua un noureau traitement avec 70 livres de sulfate de fer, ce qui porta à 240 livres la dose employée.

Les places, où la mousse noircie et desséchée était morte, se sont peu à peu couvertes de nouvelle 
herbe, qui a complètement remplacé la mousse détruite. Les racines des graminées, débarrassées de l'espèce de feutre qui les courrait, semblaient passer de l'inertie à l'actirité.

La mousse qui ne séjourne pas trop longtemps sur la terre, n'a donc qu'un effet superficiel : par l'occlusion du sol, elle empêche les racines d'émettre des jeunes pousses, mais ne les tue pas immédiatement. Aussi, l'emploi des charrues émousseuses donne-t-il sourent d'excellents résultats ; en grattant la surface du sol, elles le débarrassent du courert des mouss ss, et les racines sous-jacentes des herbes peureut alors produire de nouvelles tiges.

Mais aucune charrue émousseuse n'est capa- ble d'un effet aussi exact, aussi mathématique que celui du sulfate de fer.

Sous son action, toutes les parties mousseuses, même les plus tenaces, se trourent noircies et séchées; il décourre sous les plantes encore riraces les taches cryptogamiques, dont on ne soupçonnait pas la présence, et partout il s'attaque au parasite en respectant absolument les plantes utiles. Jamais machine à découper n'aura, dans un tissu, séparé plus exactement la broderie du fond; jamais épierreuse n'aura débarrassé avec 


\section{$-131-$}

plus de soin le grain de ses impuretés; il fouille tous les recoins de la prairie, et pas une place attaquée ne lui échappe. Son action enfin semble merveilleuse, tant elle présente de sûreté dans le résultat et d'intelligence dans son mode d'opérer.

L'essai a été comparatif: une partie égale non traitée a été laissée comme témoin; elle est restée infestée de mousse. Dans la partie sur laquelle on a opéré, les places anciennement couvertes de mousse ont été, bion avant la fenaison, totalement engazonnées d'une herbe fine et serrée.

Après le fauchage, le résultat pratique a complètement répondu à l'aspect extérieur: on a récolté sur la partie traitée au sulfate de fer, un poids d'herbe correspondant à 140 bottes de foin à l'acre, tandis que la partie non traitée domnait 81 bottes $\frac{1}{2}$.

En même temps que M. Michiels procédait à ces essais, il les signalait à M. Lambin, le sarant et sympathique professeur de la Société d'horticulture et de petite culture de Soissons, et le priait d'en faire exécuter d'analog'ues dans le jardin de la société.

Ces essais, faits arec la même dose de 135 à 200 livres à l'acre, ont domné des résultats tout à fait remarquables. L'un des derniers bulletins de 
cette société publie une conférence de M. Lambin, qui en rend compte dans les termes suirants:

"Dans certains terrains et quoi qu'on fasse, la mousse, ce fléau des prairies, ruine et appaurrit rapidement le gazou. L'un des agents les plus actifs pour s'en débarrasser est, saus contredit, le sulfate de fer employé à la dose de 400 à 600 kilogrammes à l'hectare (135 à 200 lirres à l'acre). Cette année, nous arons obtenu ici des résultats rraiment surprenants, non seulement pour la destruction de la mousse, qui a été complète, mais encore pour la régétation de l'herbe, qui a été incomparablement vigoureuse, ut cela grâce à l'emploi du sulfate de fer. Nous ne saurions trop recommander l'usage de ce sel, d'autant plus que son prix n'est pas élevé, 7 franos les 100 kilogrammes (ici à Québec. il faut lire: un centin la livre, en prenant un bari] de 285 livres).

Pour obtenir tout son effet, il faut semer en mars (ici daus la prorince de Québec, c'est le mois d'arril qui convient le mieux), en choisissant une journée plurieuse."

Les résultats obserrés à Soissons ont donc été identiques à ceux notés par M. Michiels, quoique rraisemblablement les circonstances fussent différentes. Comme ceux de M Michiels, ils confirment ceux obtenus par M. Griffiths. Ces 
résultats apaisent en même tempss les craintes manifestées sur l'emploi du sulfate de fer en agriculture. Non seulement, sous l'action à'une dose de 170 à 225 livres à l'acre, les plantes n'ont pas souffert, mais elles ont grandement prospéré, le rendement de la prairie ayant été presque doublé.

Nous ferons remarquer, toutefois, qu'une dose aussi élevée peut aroir quelques inconvénients généraux, celui, par exemple, de rendre le sol trop acide et de faciliter le déreloppement des prêles et autres plantes des terrains où l'acide domine. Le but à atteindre semble être la neutralité du sol, la présence des mousses paraissant être l'apanage des terrains trop alcalius, puisque la potasse favorise, dit M. Griffiths, le déreloppement des végétations cryptogamiques.

Aussi, nous bornons-nous à recommander seulernent les doses de 150 à 200 livres environ à l'acre, en nous appuyant sur le succès que nous renons de signaler, l'emploi du sulfate de fer daus ces conditions ayant détruit les mousses et presque doublé le rendement en foin.

Il existe encore un autre moyen de débarrasser les prairies de la mousse qui les infeste. Ce moyen consiste à herser en automne, tout en employant le tan épuisé des tanneries. 
XIV.--L'HERBE.

Pour indiquer les direrses qualités que doit aroir l'herbe, nous ne pourons faire mieux que de citer une partie de l'ezcellente conférence, que M. James Fletcher, botaniste des Fermes expérimentales de l'État, a donnée à la troisième convention annuelle de la Société d'A griculture du Canada, teune à Montréal le 6 février 1894. Cette conférence, qui porte le titre que nous avons mis en tête de ce chapitre, se lit comme suit:

"L'importance de l'herbe n'a jamais été aussi bien démontrée que de nos jours.

"Ce qu'on demande d'abord à une bonne herbe, c'est de donner une forte récolte, de manière à ce qu'elle soit payante. En second lieu il faut qu'elle soit d'une résistance au froid proportionnée au climat. En troisièmelieu, de bonnes herbes doivent être nourrissantes, c'est-à-dire riches en albuminoïdes ou éléments producteurs de viande et paurres en ligneux, matière dure et indigeste. Enfin, en quatrième lieu, elles doivent être appétissantes, pour être recherchées du bétail.

Nous trourons, sous tous ces rapports, de gran des différences entre les différentes variétés d'herbes. Dans certaines circonstances données, certai- 
nes herbes réussissent bien; dans d'autres circonstances, elles ne sont pas payantes. Il faut donc que le cultivateur réfléchisse à ce qu'il doit faire, et n'aille pas prendre la première espèce venue, que lui offre le grainetier, parce qu'il l'a sous la main. Les gens se plaigent des mélanges des grainetiers ; quelques-uns ront jusqu'à dire qu'ils ne sont bous à rien ; mais c'est aller trop Ioiu. Quelques-uns des mélanges d'herbe offert par les grainetiers sont réellement excellents et la plupart d'entre eux réussissent bien; mais il faut aussi reconnaitre qu'un bon nombre contiennent des espèces d'herbe, auxquelles notre climat ne convient pas du tout. Le commerce des grainetiers est de satisfaire aux commandes ; les commandes doirent être basées sur l'expérience des cultivateur's ; ceux-ci doivent saroir ce qui leur con rient, et, lorsqu'ils le savent, les grainetiers sont heureux de le leur fournir. Jesuis d'aris que la plupart des renseignements dont ils ont besoin pour faire un choix de bonnes espèces, les cultivateurs peurent se les procurer dans des établissements tels que les fermes expérimentales du Canada. Les expériences que nous y faisous sont entreprises dans l'intérêt de tous les cultivateurs du pays. 
suivi à Ottawa en faisant ces expériences. Nous arons pris une pièce de terre de qualité ordinaire moyenne et l'arons dirisée en parcelles d'une perche carrée chacune, chaque parcelle étant destinée à une seule espèce d'herbe. Dans d'autres parcelles, nous avons fait des essais de mélanges, $\mathrm{y}$ compris ceux des grainetiers. Ces parcelles ont beaucoup attiré l'attention des cultirateurs qui ont risité la ferme, l'été dernier.

"Nous prenons soigneusement note de tout et recueillons ainsi de précieux renseignements, tels que: le rendement en poids à l'acre de telle herbe donnée; sa valeur nutritive d'après l'analyse chimique, afin de roir si nous pourons en recommander la culture. Nous la faisons aussi consommer au bétail, non seulement pour nous assurer qu'elle est appétissante et que le bétail en est friand, mais encore pour rérifier son degré de digestibilité. Ce sont là autant de choses importantes abondantetes. Si une herbe ne donne pas une récolte assez pour qu'il soit profitable de la cultiver à quoi nous sert qu'elle soit rustique; si elle ne pousse pas, elle ne nous sert à rien; chacun de ces deux points est aussi important que l'autre.

"Ces expériences nous ont rérélé que beaucoup d'herbes indigènes méritent d'être culti- 


\section{$-137-$}

vées et nous ont prouvé que certains mélauges des grainetiers sont assurément avantageux aux cultivateurs; mais il leur sera toujours plus arantageux d'acheter les graines séparément et d'en faire le mélange eux-mêmes. Nous arous aussi trouvé qu'il y a nombre de variétés recommandées en Europe, qui n'ont pas de valeur pour nous, parce qu'elles ne résistent pas assez longtemps Nous en arions introduit un grand nombre dans les mélanges pour prairies permanentes, et comme résultat, une forte proportion de ces herbes, qui se font remarquer dans le pâturage la première année, disparaissent la seconde ou la troisième année après le semis. En Ecosse, il n'y a peutêtre pas d'herbe considérée comme ayant plus de valeur que le Ray-grass d'Italie ou vivace; il y donne de 3 à 5 récoltes sur certaines fermes, mais pas ici. Le Ray.grass vivace, à Ottawa, du moins et dans quelques autres localités, n'a pas résisté au premier hiver. Ce fait est important à counaitre, puisque cette herbe formait généralement cinquante pour cent en volume de tous les mélauges d'herbes, que rendent les grainetiers. J'apprends de M. Ewing qu'il ne met plus maintenant de Ray-grass vivace daus ses mélanges Nous pourons cependant cultiver ici avec succès et profit quelques-unes des meilleures espèces d'herbes 
d'Europe. J'en mentionnerai deux auxquelles j'accorde une grande importance: la Fétuque des prés et le Dactyle pelotonné (Orchard grass); la pre. mière derrait figurer dans les mélanges pour paturages.

"La Fétuque des prés et le Dactyle pelotonné sout deux espèces d'herbes à introduire, à mon avis, dans tous les mélanges d'herbes cultivées, au moins dans cette partie du pays.

"Le Dactyle pelotonné a une valeur spéciale à canse de la rapidité avec laquelle il repousse après avoir été coupé ou fauché. Il se plait surtout daus les terres riches, plutôt humides.

"Dans les districts montagneux, comme les cantons de l'Est, dont le climat convient peut-être mieux à l'industrie laitière que celui d'aucune autre partie du Cauada, arec une atmosphère toujours fortement chargée d'humidité, une merreilleuse abondance d'eau, et la nature accidentée ảu sol, le Dactyle pelotonné a été essayé par beaucoup de cultivateurs arec les meilleurs résultats. Fané, il fait un foin un peu dur et léger de poids; mais ce défaut est plus que compensé par ses bonnes qualités. Il fait un précieux mélange arec le trèfle et rient à maturité presque en même temps que le trèfle rouge ordinaire. Vous sarez qu'un des arguments sérieux contre 
le mélange de mil et de trèlle, est qu'il est difficile de trouver une variété de trèfle qui arrive à maturité arec le mil. Depuis deux aus nous avous essayé tous les trèfles portés au catalogue de tous les grainetiers de France, d'Allemagne et d'Angleterre. La plupart des graines d'herbes rendues en re pays viennent d'Europe, et: cela parce que nos grainetıers sout incapables de se les procurer dans le pays. Il est peu de grainetiers qui aient d'assez grandes fermes pour récolter leurs graines, c'est pourquoi il sont obligés de les importer. Ce serait une bonne chose pour les cultivateurs de produire pour le marché d'autres graines meilleures et mieux adaptées à notre climat.

"J'ai cultivé la même espèce d'herbe, de graine importée d'Ecosse, d'Angleterre et de France, et en même temps de graine récoltée dans différentes parties du Canada, et il y a u u arantage bien apparent à cultiver des graines obtenues daus motre pays. En somme, les plantes sont meilleures par le fait qu'elles commencent à s'acclimater; cette influence de l'appropriation aux circonstances environnantes se fait sentir sur tout l'ensemble de la question. Un autre point en fareur de la graine récoltée en Camada, serait qu'on couperait ainsi court à l'importation de nouvelles mauraises $h$ erbes. 
"Il est pris note de tout le trarail fait à la ferme et ces notes sont conserrées pour l'informa. tion des cultivateurs qui peurent en aroir besoin.

"En ce qui concerne les mélanges commerciaux des grainetiers, nous arons trouvé, comme je l'ai déjà dit, que beaucoup d'espèces qui y figurent disparaissaient après la première année, notre climat ne leur convenant pas; mais il restait un nombre suffisant de rariétés pour garantir leur emploi au cultirateur, et, si j'ai un conseil à donner à ceux qui reulent semer des graines d'herbes, soit pour prairie, soit pour pâturage, c'est de choisir eux-mêmes les espèces qui leur conviennent et de faire le mélange eux-mêmes. S'ils ne connaissent pas les meilleures espèces, qu'ils m'écrivent. à Ottawa ou qu'ils consultent les grainetiers : car ceux-ci ont intérêt à trourer ce qui convient à leurs clients; peu leur importe ce qu'ils vendent, pourtu qu'ils donnent satisfaction. Dites-leur ce dont rous arez besoin, quel est rotre sol, le but auquel rotre récolte est destinée, et n'ayez aucune crainte de comsulter les grainetiers ; il n'y a aucun antagonisme d'intérêt entre eux et rous; rous avez besoin les uns des autres."

Maintenant que nous renons de roir les direrses qualités de l'herbe, nous allons jeter un 
coup d'œil sur sa haute utilité et l'emploi que l'on en fait.

L'herbe est utilisée de deux manières: verte, dans les champs, elle sert au pâturage des animaux de la ferme, principalement du bétail ; séchée, à l'état de foin, elle est la nourriture de tous les animaux ruminants pendant l'hirer.

Nous allons done examiner l'herbe sous sa forme naturelle, sur pied, dans les champs, sous forme de pâturag'e ; ensuite, à l'état de foin.

Importance d'Un bon Paturage. - La meilleure nourriture que l'on puisse donner au bétail, principalement aux raches laitières, c'est la boune herbe des champs, sous forme de pâturagee. L'horbe fratche est un aliment doux, succulent, riche en principes alibiles (propres à la nutrition), des plus faciles à digérer et par conséquent des plus assimilables.

Prenez deux raches à lait de même taille et de mêmes aptitudes; nourrissez l'une dans un bou pâturage, et l'autre arec du fourrage vert, à satiété, ou même d'un mélang'e d'aliments quelconques; rous obtiendrez beaucoup plus de lait riche de la rache nourrie daus un bon pâturage 
que de celle à laquelle rous donnerez la même quantité de fourrage rert, sans compter que la première aura plus beau poil, ce qui annonce un bien meilleur état de santé. Le lait de la rache nourrie au pâturage donnera un beurre d'une couleur et d'une,sareur agréable, au lieu que celui de la rache nourrie exclusivement au fourrage vert fauché, ou de quelque autre fourrage que ce soit (stabulation permanente), ne produira qu'un beurre de qualité inférieure.

Le pâturage est la nourriture la plus naturelle, la plus facile et la plus économique du bétail. En Suisse, on estime qu'une prairie qui peut nourrir trois raches au pacage, ne peut en nourrir que deux, si elle est fauchée. Les Anglais croient aussi qu'une prairie pâturée fournit plus de substance alimentaire que celle fauchée deux fois. Les premières pousses sont plus nutritires que les suivantes. Block estime à 8 pour cent cet excédent de valeur nutritire. Dans les pays de produits laitiers, comme la Suisse, la Hollande,le Danemark, la Normandie, les vaches sont nourries presque entièrement d'herbe, et elles donnent de grandes quantités d'excellent lait.

En outre, lorsque l'herbe a été broutée, clle 
croit immédiatement arec nue plus grande rapidité. L'herbe d'une prairie est coupée presque tous les jours par les dents de l'animal qui y pâture, tandis que, si elle est fauchée, elle n'est coupée qu'une ou deus fois dans le cours d'un été.

QUALITÉ NUTRITIVE DE L'HERBE DES PRÉS.D'après le docteur Kühn, une herbe est d'autant plus nutritive qu'elle est plus jeune, qu'elle a été plus fortement fumée et qu'elle est renue dans des terrains plus riches. Cela étonnera beaucoup certaius éleveurs qui s'imaginent que le foin de première coupe est plus fortifiant que le regain. D'après le même docteur Kühn, une plante très déreloppée et arrivant vers la fin de sa croissance dans un terrain pauvre ou médiocre et peu fumée, ne vaut pas à beaucoup près une plante jeune, élerée dans de bonnes conditions. La jeune herbe de prés arrosés de puriu, le jeune trèfle, pâturé ou fauché arant la floraison, le regain des prairies fumées sont les fourrages par excellence.

Les feuilles et les fleurs sont ordinairement deux ou trois fois plus riches que les tiges, daus une plante coupée à la floraison, et c'est parce qu'ils font surtout développer les feuilles que les engrais azotés accroissent la richesse en protéine. Ein effet, les engrais azotés produisent une végétation luxuriante, riche en matière grasse et en protéine. 
En général, les herbes sont d'autant plus riches en principes alimentaires, protéiques surtout, qu'elles sont plus feuillues, plus fines, moins ligneuses et croissent dans un sol mieux fumé en engrais azotés. Elles sont plus riches dans les années sèches que dans les années humides, dans les sols fertiles ou secs que daus les sols paurres ou marécageux.

Isidore Pierre ajoute que pour chaque espèce de fourrage le regain est beaucoup plus riche en matière azotée que le fourrage en pleine floraison, et que la différence est à peu près comme du simple au double.

La différence entre les regains et les fourrages plus déreloppés de la première coupe de l'été, résulte de ce que les premiers sont plus riches en feuilles, par suite, plus riches de 30 à 40 olo en matières azotées.

Nous croyons pouvoir ajouter que les regains sont supérieurs aux foins ordinaires parce qu'ils renferment moins d'insectes de toutes sortes.

Forn.-Le mot foin est un nom générique par lequel on désigne les plantes herbacées, coupées avant la maturité et desséchées pour l'alimentation des animaux.

De même que l'herbe rerte des pâturages est la nourriture la plus naturelle du bétail, de 
mème le foin constitue aussi la nourriture la meilleure, la plus économique et la plus arantageuse que l'on puisse donner au bétail en hirer.

A u printemps, de bonne heure, le bétail aime et recherche arec avidité l'herbe très jeune et très tendre. Au milieu de l'été, il mange assez volontiers l'herbe fleurie, c'est-à-dire un peu plus dure, et à mesure que l'automne arance, il mange avec goût les herbes les plus dures et même mûres.

$\mathrm{Si}$, pendant l'hiver, on fait sortir les vaches, elles n'ont rien de plus pressé que de chercher les quelques brins d'herbe séchée qui ont échappé à la faux.

L'herbe verte pour l'été, l'herbe séchée ou foin pour l'hiver, telle est la loi de la nature qu'il faut suivre pour l'alimentation du bétail, comme en toute autre chose.

Le foin est un aliment naturel, qu'aucun autre ne peut remplacer avec avantage.

Dans le bon foin, les principes les plus essentiels - les matières azotées, les corps gras, les glycosides (sucres), les substances salines-se trouvent dans les proportions les plus propres à flatter le goût des animaux, puisqu'ils ne s'en lassent jamais, et les plus favorables au développement du corps animal comme à l'entretien de la santé. En 
effet, de tous les aliments, c'est celui qui convient le mieux aus herbirores; c'est même le seul qui, administré exclusirement, peut les entretenir indéfiniment en bon ètat.

L'HERBE ET LE FOIN, FOURRAG ES À ENCOURAGER.-Trop sourent, les primes attribuées aux animaux ne sont que des primes données à un effet, sans récompense pour la cause qui le produit. L'étât des animaux en effet, n'est que la conséquence de la production, de la multiplication du fourrage.

Si l'on domnait des primes pour eucourager cette multiplication, ce serait, il nous semble, bieu plus logique que de ne les donner qu'aux animaux, qui ne sont qu'un résultat. A la rigueur, on n'aurait pas méme besoin de primer les animaux, si l'on primait les fourrages au point de rue de leur multiplication; car, en faisant des fourrages, on serait rigoureusement forcé d'augmenter son troupeau, pour les consommer.

"Il ne faut pas se méprendre, dit Richard, Dictionnaire d'Agriculture: les animaux ne sont qu'un effet, qu'un résultat de la quantité des pro. duits dont on les alimente et des soins constants dont on les entoure. Si les produits sont riches et abondants, les animaux qui en résultent sont beaux 
et forts; si au contraire, il sont maigres et rares, si on lésine sur la quantité de nourriture qu'on leur donne, les animaux seront petits, chétifs et maigres comme les aliments qui leur ont été administrés arec une parcimonie forcée,

"Pour arriver pratiquement à l'amélioration du bétail, qu'on ne l'oublie pas, il faut songer à améliorer le sol qui améliorera ses produits directs; le perfectionnement et maintien des animaux en seront une conséquence directe. Si vos vég’étaux ne sont pas d'une espèce supérieure, il faut s'en procurer ailleurs ou d'autres variétés.

"Il ne faut pas non plus oublier que le climat et les influences de la localité ont des conséquences sur les animaux. On ne devra pas croire qu'en transportant sur nos sables secs des raches de la Flandre française, on leur fera produire du lait en aussi grande quantité que là où elles sont níes. Petit à petit ces raches prendront le caractère des races de leur nourelle patrie. On luttera vainement contre cette action de la nature des lieux, elle s'opérera malgré rous, parce qu'elle est la conséquence d'une loi naturelle immuable. A force de science et d'art nous pourons quelquefois modifier les effets de cette loi : un agriculteur intelligent étudie ces eflets et sait en faire une heureuse 
application au but qu'il doit se proposer et qui est toujours subordonné à ses ressources. Que d'éléreurs peurent échouer après bien des efforts et des dépenses, s'ils ne tiemnent pas compte des conditions agricoles ou climatériques dans lesquelles ils opèrent. Ne croisez donc pas des types importés mal adaptés aux animaux que rous arez céjà. Si rotre race est bonne, le meilleur moyen de lacon. server, c'est de la perfectionuer par elle-même, sauf quelques exceptions bien étudiées. Lorsqu'el. le a besoin d'être modifiée pour une raison quelconque, choisissez des reproducteurs qui se rapprochent le plus du type que rous arez déjà et améliore z le régime alimentaire. La nature ne vous donuera qu'en raison de ce qu'elle recevra"

On ne pourrait donc pas assez insister sur les arantages que le cultirateur canadien retirerait, s'il se li rrait à la eulture de bonnes prairies.

Les cercles agricoles ne derraient pas manquer d'appliquer la plus grande partie de leurs octrois à encourager de toutes leurs forces l'établissement des bonnes prairies.

Nous espérons aussi qu'ils accorderont une prime pour la meilleure prairie. 


\section{$-149-$}

XV.-Exploitation ET PRODUIT DES PRAIRIES.

Il nous paraft incontestable, et c'est un fait d'observation pratique, que le produit des fourra ges artificiels va constamment décroissant depuis un certain nombre d'années; non seulement le produit annuel est moindre, mais encore la durée du plant ra toujours diminuant. Tandis qu'une luzernière bien établie et bien entretenue peut durcr et durait dix, quinze, vingt ans et plus, sa durée moyenne n'est plus guère aujourd'hui que de cinq à six ans; encore, les dernières récoltes laissent-elles souvent à désirer dans le produit en fourrage. Il en est de même aussi pour le sainfoin qui, de six à huit ans de durée, est descendu à trois ou quatre. Irart, en 1809, disait encore que la longevité naturelle du sainfoin peut aller, dans quelques cas jusqu'à $2 \nmid$ ans et peut-être même au-delà, ainsi que l'atteste un fait cité par M. Bonneau; et celle de la luzerne, à trente ans, comme il s'en est conraincu lui-même. Et il en est de même pour toutes les autres herbes fourragères.

La première et la principale cause de cette dégénérescence, c'est l'feffilement (épuisement) du sol et du sous-sol par la culture des mêmes plantes pendant un temps trop long ou à intervalles 
trop rapprochés. Ceci"s'applique surtout aux herbes fourragères cultirées sfules, sans mélanges. Outre cette cause, il en est d'autres encore que nous derons signaler ici.

Alternatice des modes d'exploitation.De même que le régime d'un animal doit être aussi rarié que possible, que les engrais rendus au sol doirent être alternés dans leur composition, que l'alternance des récoltes est une des lois de la nature, de même aussi le mode d'exploitation des prairies doit alterner par le fauchage et le pâturage. Le fauchage, coupant la plante assez près de son collet, farorise la repousse de nouvelles tiges ; le pâturage tasse et raffermit le sol, qu'il enrichit en outre de quelques engrais. Mais tous deux doirent être surveillés dans la pratique, sous peire de devenir préjudiciables: le fauchage ne sera exécuté ni trop haut ni trop près de terre; le pâ. turage se fera seulement par les temps secs, en excluant les poulains, les cheraux et surtout les moutons, c'est-à-dire en n'admettant que les bêtes à cornes.

Puisqu'il faut faire alterner le fauchage et le pâturage, il faut donc toujours combiner les mélanges de plantes fourragères de manière à avoir une coupe (prairie) et un pacage (pâturage). De fait 
lune bonne prairie doit donner, outre une coupe abondante de foin, un bon pâturage pour le bétail ; et tun bon pâturage, outre le pacagie qu'il doit fournir au bétail, doit donner une boune coupe de fourrage.

On arrivera à ce double résultat, d'abord en employant les mélanges indiqués dans cet ourrage, ensuite en fauchant et faisant paitre alternativement les prés ainsi ensemencés.

FAUCHAGE DES PRAIRIES TOUS LESANS--Lorsque les prairies sont fauchées tous les ans, elles devienment plus sujettes à produire des mau raises herbes. En effet, disent certains praticiens, le fauchage des prairies chaque année farorise la pousse de la mousse et des mauraises herbes de toute espèce, tout particulièrement celles à fortes racines, qui changent graduellement et détruisent la nature et la qualité de l'herbage; le trèfle blanc disparait et les plantes grossières se disputent le terrain avec les bonnes plantes fourragères.

En opérant comme il a été dit au précédent paragraphe, ou est donc à l'abri d'un pareil état de choses.

Prairies et pâturages. - Au point de vue du maintien de la fertilité du sol, le fauchage et le pâturage n'agissent pas de la même manière. Le 
pâturage, surtout s'il est alterné avec le fauchage, conserve indéfiniment l'herbage daus son état de fertilité; il l'améliore même.

Au contraire, le fauchage, seul, tend à appaurir darantage le sol, et tout particulièrement si l'ou rend tout le foin au lieu de le faire consommer sur la ferme; car alors le cultirateur n'a pas autant a'engrais à disposer pour la bonne tenue des prairies. L'expérience a démontré que pour conser. rer d'une manière presque permanente le même degré de fertilité aux prairies fauchées, en moyenne, il leur fallait la moitié de l'engrais résultant de leur produit en foin.

ALternat des PATURAGEs.--L'époque oì l'on doit livrer la prairie au pâturage doit varier arec la température, le sol, la disposition du terrain, les herbes dont elle se compose, le mode d'exploitation du domaine, etc; tantôt on se trourera mieux de faire paitre au printemps, tantô: l'automne sera préférable.

En général, cependant, il raut mieux faire pâ. turerles prairies rers le commencement de juin, faucher à la fin de juillet ou au commencement d'août; alor's le foin est de qualité supérieure, ayant retenu les meilleures plantes, parce que les sécheres. 
ses de l'été ont détruit une partie considérable des mauvaises herbes au profit des bonnes. Pour pâturer une prairie que l'on doit faucher dans la même saison, roici comment l'on opère. On met dans le champ autant de têtes de bétail (principalement des jeunes animaux) qu'il en faut pour que l'herbe soit totalement rasée en huit à dix jours. Plus on fait raser l'herbe nettement, plus elle repousse vite et touffue. Toutefois, il faut veiller à ue pas laisser brouter l'herbe trop près de la racine, ce qui cauzerait un tort grave aux prairies en détruisant les racines.

On changera le bétail d'enclos assez sourent pour éviter que l'herbe ne soit rasée trop près de la racine, ce qui lui ferait grand tort, et aussi afin que l'odeur des déjections du bétail dispraraisse.

XVL. Fauchage des prairies. -La fenaiSON.

"Avant d'enroyer les faucheurs ou les faucheuses mécaniques, dans un pré, dit dans un excellent article le sarant M. Gayot, on a dî s'assurer que le moment farorable était renu de couper l'herbe pour la convertir en foin de bonne qualité. Quelle est donc la phase de végétation qui offrè à ce point de vue le plus d'arantage à la pratique? 
Celle, à n'en pas douter, où la plante, après avoir déreloppé à leur maximum toutes ses feuilles, ouvre aussi toutes ses fleurs. C'est alors, en effet, qu'elle possède, aussi également répartis que possible, dans toutes ses parties, les principes alimentaires qui lui sont propres, et qu'elle peut les offrir à l'animal dans leur plus grand état de perfectionnement pour la nutrition.

"Arant la floraison, les plantes renferinent trop d'eau de régétation; après ia fleur, la vie du régétal se concentre trop exclusirement dans un seul fait, la fructification. Alors, le reste de la plante se dessèche et devient cassant; les manipula. tions du fanage, celles qu'entrainent le trausport, l'emmagasinement, le bottelagee, font tomber et les feuilles et les graines; les tiges dures et ligneuses restent seulns et ne constituent qu'un fourrage peu recherché et peu nutritif." (Encyclop-piat. de l'Agric, art Fenaison). Faucher trop tard, c'est le tort général des cultirateurs canadiens, et il faut une surreillance bien actire pour ne pas laisser passer le moment précis qui seul peut domner du foin saroureux et aromatique. Ecoutons plutôt M. de Dombasle: "On peut remarquer, dit-il, qu'en général, on est disposé à faucher trop tard; on croit gaguer en quantité, et l'on perd beaucoup plus sur la qualité du foin. Le moment de faucher une 
prairie est celni oì les plantes y qui abondent le plus, et qui produisent le meilleur fourrage, commencent à fleurir ou au plus tard sont en pleine fleur. Lor'squ'elles sont à ce point, quelques jours de retard font une différence très considérable dans la qualité du fourrage, car toute plante qui a amené ea grainu à maturité ne produit plus qu'un foin dur, peu savoureux et pen nourrissant pour le bétail; et les meilleures plantes des prairies, principalement les graminées les plus précieuses, passent arec une rapidité étonnante de.la floraison à la maturité." (Calendrier du bon Cultivatent; Te édition, p. 190.)

"Il y a cependant une exception à cette règle générale, continue M. Gayot, exception tirée de la nécessité de travailler à l'amélioration du produit de certaines prairies assises sur un fonds humide, qu'on n'est pas disposé à assainir par un moyen plus expéditif et plus efficace. Alors, il faut faucher de bonne heure, avant l'époque que nous avons soigneusement déterminée plus haut, car en les dépouillant ou en expose la surface à toute l'intensité des rayons solaires qui en opèrent le desséchement et permettent de recueillir, à la seconde coupe, des plantes qui ont poussé dans des circonstances plus favorables. D'ailleurs, dans ce cas, les végétaux propres aux lieux humides dépérissent 
sous l'influence d'une humidité moindre, et cèdent la place à d'autres dont la sorte et les qualités alimentaires, très supérieures aux premiers, produisent aussi de meilleurs résultats quant à la uutrition.

"Il faut encore que l'on sache ceci, par exemple: Les plantes parasites ou nuisibles, malheureusement si nombreuses dans les prairies permanentes, sont généralement consommées arec impunité dans les premières phases de leur régétation. Les principes doux, aqueux, mucilagineux, inertes, prédominent alors, tandis que la naturité déreloppe les sucs âcres, amers, narcotiques, rireux, qui les rendent dangereuses. C'est un autre motif à inroquer en faveur des fenaisons un peu précoces." (Ut supra.)

En général, c'est à la în de juin ou au commencement de juillet qu'on doit commencer la fauchaison, quelquefois plus tôt, quelquefois plus tard, suirant que l'aunée est sèohe ou plurieuse; les prés hauts sont les premiers mûrs, puis viennent les prairies moyennes, ensuite les prairies arrosées, en quatrième lieu les prairies basses, et enfin les prés tourbeux et marécageux. Cependant, cet ordre n'a rien d'invariable et peut être influencé par l'exposition, les engrais, etc. 
Ainsi, une prairie fumée en couverture au printemps, devient par cela même un peu plus tardive que les années précédentes; à sol et à pentes égaux, un pré exposé au nord est moins hâtif que celui exposé au sud. L'essentiel est de se guider sur la floraison des plantes principales et les plus nombreuses

Nous arons done mis la faux dans l'herbe: "On doit apporter une grande attention an tratravail des faucheurs, pour qu'ils fauchent le plus près de terre qu'il est possible; un pouce de longueur près de terre produit bien plus de foin que plusieurs pouces au haut des tiges, parce que l'herbe y est bien plus garnie : c'est pourquoi l'on éprouve une perte considérable dans le fauchage des prés où le sol n'est pas bien uni, où l'on a négligé d'étendre les taupinières et les fourmilières, où I'on a laissé des pierres, etc." (De Dombasle, comme ci-dessus, p. 190.)

Fenaison.- Le temps le plus propice pour la coupe de l'herbe est celui où toute la rosée est disparue. Il faudra naturellement, au préalable, commencer à examiner soigneusement l'état du ciel, afin de s'assurer si, d'après les signes ordinaires, bien connus de tous les enltivateurs, on va avoir quelques journées de temps séchant. On commencera donc à faucher vers dix heures du matin. On 
éparpillera le foin à mesure qu'il sera coupé, puis on le retonrnera rers deux heures de l'après-rnidi.

Plus le foin est étendu également et mieux il est dirisé, plus la dessiccation s'opère prompternent et régulièrement.

Isa rosée, comme la pluie, fait blanchir le fuiu. Pour le soustraire à cet inconrénient, on le rassemble en tas (reillottes) assez tôt daus l'après-midi pour que ce trarail soit acheré arant que la rosée du soir tombe, puis on courre chaque reillotte arec une courerture ou chapeau en pulpe ou en toile cirée.

Voici une très bonne manière de procéder: Prenez une fourchée de foin, soulevez la et placezla sur le terrain libre le plus roisin; soulerez•en une nourelle fourchée, puis une troisième que rous placerez les unes au-dessus des autres, de faren à leur domner un bon appui. Si le foin a séché contenablement dans la journée, on pent entasser l'équiralent de cinq à six bottes par reillotte; ce sera trop pour du foin encore fort humide. La reillotte ne derrait jamais, mesurer plus de cinq pieds de diamètre à sa base. Ainsi faite, on aura le soin d'en faire le tour battant le foin de la jambe et de la fourche, de manière à la mettre antant que possible à l'abri du maurais temps. 
Cela fait, on apportera les couvertures ou chapeanx en pulpe ou en toile cirée, et on en courrira; la veillotte ainsi courerte est à l'abri du vent, ne peut plus se défaire, mème dans les forts coups de vent, et lapluie la plus abondante s'écoulera à l'extérieur, sans la pénétrer. Il est important de ne pas placer les tas de foin daus un trou, mais sur les planches et même sur l'endroit le plus élevé des planches. La manière indiquée pour la formation des reillottes a sa raison d'être et ne procède pas du caprice. En effet, en procédant par moyennes fourchées, le foin se superpose par couches à peu près égales, de sorte que par le foulage, la pluie n'a plus d'accès par les côtés de la reillotte et ne peut la pénétrer.

Le lendemain matin, un peu tard, on enlère la couverture des veillottes, et on laisse ainsi le foin toute la journée, afin de le préparer à se sécher plus facilement et plus rapidement, la journée suivante, de manière à ce qu'il conserre toutes ses feuilles et toutes ses fleurs, et ensuite pour amollir ses tiges par la fermentation modérée qui s'y déreloppe et peut aller jusqu'à brunir le foin, ce qui le rendra de digestion plus facile, plus nutritif, et plus agréable aux animaux, enfin de beaucoup supérieur pour la production du lait.

Le soir, on recouvre de nouveau les veillottes 
arec les chapeaux. I.e lendemain matin, vers dix heures, la rosée étant disparne, on enlèveles courertures, puis on ourre les reillottes et on étend le foin, mais seulement juste le temps nécessaire pour qu'il achère de se sécher, ce qui n'eșt pas long, si l'opération a été bien conduite. Puis, enfin, on ramasse et on rentre le foin ainsi séché et en excellent état, avant la rosée du soir.

Le foin ainsi préparé ne laissera rien à désirer sous le rapport de la couleur, de la qualité et du parfum, et c'est celui qui se cotera au prix le plus élevé.

Le plus grand nombre des cultivateurs de cette prorince ont pour habitude de laisser sécher le foiu dès qu'il a été fauché, sans le mettre en tas. Le fuin est alors plus ou moins décoloré et n'a pas l'arome qui distingue le foin de première qualité ; il se rend par suite moins facilement et moins cher.

Le procédé indiqué ci-dessus est celui suiri à la Ferme expérimentrale centrale d'Ottawa, ainsi que dans une partie de la province d'Ontario et des Etats-Unis. 


\section{XVI.-FOURRATES VERTS}

Comme nous l'avons déjà dit plus haut, le meilleur de tous les fourrages verts est la boune herbe tendre et jeune que les animaux prennent eux-mêmes au pâturage.

Mais comme la plupart de nos cultivateurs n'ont à exploiter que des terres qui ont été complètement épuisées ou par une mauvaise culture, ou par une répétition trop fréquente des récoltes de grains, et que l'herbe, une fois rasée par le bétail, refuse de repousser pendant une trop graude partie de la belle saison, il serait téméraire d'espérer une production assez forte de fromage et de beurre sous forme d'une grande abondance d'un lait riche et savoureux. Il faut donc lui renir en aide d'une manière prompte et efficace. On y arrivera par la cuiture des fourrages verts, dans le sens ordinaire de ce mot, qui comprend toutes les herbes fourragères, graminées et légumineuses, que l'on fauche de bonne heure au printemps, immédiatement avant la floraison, et dont les coupes subséquentes, faites dans le cours de l'été et au commencement de l'automne, permettent de fournir du fourrage vert au 
bétail, principalement aus raches laitières, pendant toute la belle saison, c'est-à-dire depuis le mois de mai jusqu'au mois de norembre. Ce mot sert aussi à désigner les fourrages verts dont on emplit le silo, afin que les raches aient à leur besoin, toute l'aunée, une nourriture dolice, rafraîchissante et succulente.

La culture de ces herbes ou plantes n'est pas dispendieuse, à raison du prix peu élevé de la semence, mais elle est assurément très profitable, si elle est faite avec intelligence et suirant un système raisonné, et elle pourvoit au plus grand besoin de motre industrie agricole, celui de procurer au bétail une nourriture bonne, succulente et fortifiante, pendant les mois les plus secs de l'année.

De plus, l'alimentation aux fourrages verts, en premier lieu ceux que les animaux paissent eux-mêmes au pâturage, et ensuite ceux fauchés et serris comme addition au pacage, coûte moins cher que l'alimentation aux fourrages secs consommés à l'étable, et même que les fourrag'es rerts ordinaires, mais donnés aux animaux en stabulation permanente. L'expérience générale ne per. met pas le moindre doute à cet égard.

Les meilleures herbes à semer pour fournir un fourrage vert au bétail, sont: 
Le seigle, avec trèfle rouge ;

La luzerne, arec brome inerme;

Le dactyle pelotonné, en mélangre avec du trèfle rouge;

Le sainfoin, additionné de fromental;

Le mais (blé-d'Inde) canadien.

LE SEIGLE-Chaque cultivateur devrait semer un petit champ de seigle, nous devrions dire deux ou trois petits champs; car ce grain arrive si vite à maturité, que huit ou au plus dix jours après qu'il est bon à être fauché les animaux ne veulent plus le manger.

On devrait toujours ajouter au seigle une certaine quantité de trèfle rouge. Les mélanges de légumineuses et de graminées, surtout si on le fait arec soin et que l'on associe des plantes rivaces (trèfle rouge) d'un côté, et des plantes annuelles (seigle, blé) de l'autre, sont fort productifs.

On sèrne le seigle ordinairement sur un retour de chaume, après avoir donné une ringtaine de charges de fumier ou 200 lirres de sulfate d'ammoniaque à l'acre; on herse jusqu'à ce que la terre soit bien émiettée, et l'on y passe le rouleau pour achever la besogne. 
Il faut toujours semer le seigle à raison de trois minots à l'acre. Quoique la graine en soit petite, cette quantité de semence n'est pas trop forte, car plus on le sème fort, plus vite on pourra le faucher, et ce point est important quand il s'agit de nourrir des raches à lait au fourrage vert.

Quand on sème le seigle à l'automne, ce qui est beaucoup mieux, attendu qu'il peut domner une coupe alors que l'on ne riendrait que de confier la semence à la terre, si l'on arait attendu le printemps pour faire les semailles, il faut enterrer la semence à trois pouces de profondeur, chose peu difficile à faire arec un drill-semoir d̀ rang's. Si rous n'arez pas de semoir, il rous faudra l'enterrer à la charrue, en tirant de très petits sillons. Tout l'ourrage pour l'ameublissement du terrain derra être fait arant l'enterrement du grain, de sorte qu'il n'y aura pas besoin de herser ni de rouler après la semaille. A u printemps suivant, le seigle profitera plus vite après un petit coup de herse et le passage du rouleau.

Il serait arantageux de remplacer un minot de seigle par un minot de blé. Quoique le blé ne profite pas aussi rite que le seigle, le rendement en serait plus copieux, surtout rers la fin de la saison, et l'on sait bien que tous les animaux de la 
ferme préfèrent le blé en vert à n'importe quel autre fourrage.

La quantité de semence que nous conseillons peut paraftre trop grande, mais soyez assurés que si vous épargnez la semence en cultivant l'herbe ou le fourrage vert, vous n'aurez que très peu de profit quand l'heure du fauchage arrivera. En Angleterre, on sème toujours quatre minots de seigle d'automue à l'acre, et l'on trouve non seulement que la masse récoltée en est plus forte, mais encore que la végétation en est beaucoup plus précoce, chose très importante pour obtenir un bon fourrage vert de bonne heure le printemps.

Au printemps, le champ de seigle doit être semé à deux reprises, séparées par un intervalie de quinze jours. Un arpent de seigle, si le terrain est fertile et bien travaillé, peut nourrir vingt vaches pendant dix jours, en y ajoutant un peu de bon foin. Il faut absolument laisser faner tout fourrage vert pendant plusieurs heures avant de le faire manger aux animaux, à moins de le mélanger avec du foin haché, ce qui raut encore mieux. Au commencement de la saison, il ne faut employer ce fourrage vert que peu à peu, après que les animaux auront fini leurs repas et auront la panse bien remplie, ou bien encore le mélanger avec du foin, comme ci-dessus. 
Ponr les chevanx, quand le printemps arrive, ils rous sauront gré d'une bonne botte de seigle par jour. Après aroir passé un long hirer, sans goûter ni carottes ni choux de Siam, les chevaux commencent à se fatiguer d'une nourriture toujours sèche, et quand même les paurres bêtes seraient obligées de reprendre bientôt leur ancien repas de foin, etc, le changement opéré par quelques bottes de seigle leur procurera un rafraîchissement bienfaisant.

Le seigle pèse 56 livres au minot; le blé et le trèfle pèsent 60 lirres au minot.

Luzerne-La luzerne, ou trèfle de Bourgog'ne, ne convient pas à tous les sols. Quant au froid, elle s'en moque assez; mais l'eau du sous-sol la tue. C'est une des plus anciennes plantes fourragères dont on ait connaissance.

Les trois coupes abondantes par saison que donne cette plante, quand le terrain et la culture lui conviement, doirent engager chaque cultirateur, dont la terre est propre à cette culture, à semer deux ou trois acres de ce magnifique green meat.

La luzerue réclame un terrain dont le soussol soit sec. Les bomnes terres franches, de sable, de glaise ou de terreau lui conviennent parfaitement; mais la semer sur des terrains d'argile o 
l'eau séjourne au printemps et à l'automne, c'est gaspiller son temps et son argent.

Après aroir choisi une pièce de terre pas trop loin du pâturage, nettoyez-la parfaitement, sans y laisser un seul morceau de chiendent. L'automne arrivé, étendez-y une bonne couche de fumier en l'enterrant aussi profondément que possible. Pas de danger de la trop enterrer, car les racines de la luzerne pénètreront jusqu'à quinze pieds et même davantage dans le sous-sol, s'il n'y a pas d'eau. On en a même vu de trente pieds dans les terres tout à fait propres à cette culture. Ce sont de bons chasseurs que ces racines.

$\mathrm{Au}$ printemps, quand la terre est devenue sèche, semez-y de l'orge, et quand la herse aura bien-mais très bien-émietté la pièce, semez-y à la volée seize livres de luzerne à l'acre, en l'enterrant au moyen de la herse-c-chaines ou d'une herse à branches. La herse à branches achève si bien et d'une manière si parfaite toutes les opérations qu'on lui confie, que nous conseillons à chaque cultivateur de s'en procurer une. Finalement, passez-y le rouleau.

L'orge une fois engrangée, une légère couche de fumier aidera à la jeune luzerne à résister aux attaques de la gelée. Une récolte protectrice d'avoine coupée en vert conviendrait encore mieux 
qu'une récolte d'orge pour grain, parce qu'elle occuperait la place moins longtemps, ce qui per. mettrait à la jeune luzerne de croitre plus rigoureusement et, par suite, de mieux résister aux rigueurs de l'hiver. Pour plus de détails voir au chapitre XII.

Le printemps suirant, il vous faudra herser la pièce avec une moyenue herse et, cela fait, vous y passerez encore le rouleau. A l'automne, après la dernière coupe, hersez énergiquement avec une herse à dents bien aiguisées, jusqu'à ce que toute mauraise herbe soit déracinée et que la terre ait l'air d'un jardin hien cultivé. Pas de danger ảe faire tort à la luzerne en la traitant de cette façon; à cette époque, les racines auront tellement péuétré le sous-sol que la herse la plus pesante ne saura les en arracher.

Une plante qui rous donnera trois coupes par été, mérite d'être bien nourrie; vous ferez donc bien de lui fournir une fumure tous les ans.

La luzerne doit être fauchée arant que la fleur s'épanouisse, et cela arrive vers la dernière semaine de mai dans les saisons ordinaires en cette province.

Le plâtre répandu au printemps sur la lu-. zerne, de même que pour les autres légumineuses, 
lui imprime une régétation vigoureuse et luxuriante.

On doit ajouter à la luzerne une petite quantité de brome inerme qui est une graminée vivace prospérant fort daus les terrains secs et riches en humus. En Hongrie, on cultive beaucoup ce mélang’e.

DACTYLE PELOTONNÉ ET TRÈFLE ROUGE MÊLÉ. - Pour suivre la première coupe de la luzerme, on ne trouvera rien de plus utile que le dactyle pelotonné, semé à raison de deux minots à l'acre et mêlé arec huit livres de trèfle rouge. Le dactyle pousse toujours en touffes, c'est pourquoi il faut y ajouter du trèfle pour remplir les lacunes laissées par le dactyle. D'ailleurs, la quantité d'azote conteuue dans le trèfle remédiera aux qualités alimentaires inférieures du dactyle. On doit semer ce mélange à la volée avec de l'orge, ou mieux avec de l'avoine destinée à être coupée en verl, au printemps, en l'enterrant au moyen de la herse à branches, après le dernier hersage donné à l'orge ou à l'avoine, et puis y passer le rouleau.

Trèfle rouge VIVACE-Cette espèce de trèfle est la plus propre à semer avec le dactyle. En l'achetant, il ue faut pas se laisser tromper par une autre espèce de trèfle rouge, le trifolium 
medium, dont la graine est meilleur marché, mais dont le produit ne vaut rien.

Ee 1889, les MII. Dawes, de Lachine, donnèrent à M. A. R. J. Fust, rédacteur du Journal of Agriculture, la permission de semer un arpent en trèfle rouge sur leur terre, et ils s'en trouvèrent fort bien. Depuis le 12 juin 1890, au dire du fermier de ces messieurs, ils ont continué à faucher ce trèfle en rert, et sans lui, leur laiterie aurait beaucoup souffert. Le 14 octobre, il y arait beaucoup de trèfle sur le champ.

Nous venons de dire qu'un des meilleurs fourrages verts pour faire produire du lait aux raches est un mélange de trèfle rougc virace et de dactyle; en roici un exemple: M. Henry Stewart, agronome distingué des Etats-Unis, confiait à un de ses amis le fait suivant:

"Quand, au lieu du trèfle et du dactyle, j'ai fait manger du mil à mes vaches, le rendement en beurre a baissé de 25 livres à 17 lirres par semaine, et je n'ai jamais pu remettre mes raches dans leur premier état de production, quelle que fût la quantité de grain et autres fourrages que j'ajoutai an mil."

D'un autre côté, nous royons dans le rapport que M. G. A. Giguult, assistant-commissaire de 
l'Agriculture, a fait de son voyage en Europe en 1894, " qu'on ne doit pas domner autant de mil que d'autres espèces d'herbes, car la sécrétion du lait en serait diminuée."

Nous lisous dans le Journul of Agricullure, numéro de juin 1894, ce qui suit:

"Mil-Pour notre part, nous roudrions voir le mil remplacé par quelques autres herbes, excepté le cas où on le cultive pour le marché : une herbe qui ne fournit pas de pâturage, qui ne domne qu'une récolte par saison, n'est guère avantageuse pour le cultirateur, surtout lorsque l'on considère que l'usage principal de ce fourrage est limité à la nourriture des chevaux; car aujourd'hui tout le monde sait, les plus routiniers même savent que le bon trèfle est bien préférable au mil pour les vaches. De fait, beancoup d'autres herbes fourragères sont non seulement tout aussi bounes, mais même bien meilleures pour l'alimentation des vaches laitières que le mil, surtout tel qu'on le vend sur nos marchés."

SAINForN--Ceux qui habitent les côtes arides du fleuve ou autres terrains semblables, peuvent s'éparguer la peine de labourer trop souvent leurs champs éloignés, en y semant du sainfoin. Une chose digne de remarque pour cette herbe, e'est 
qu'elle produira denx fois autant d'herbages pendant la troisième année de son ensemencement qu'elle en aura produit la première année.

Cultivé sur uu terrain propice, le sainfoin durera de six à dix ans Sur les côtes du nord de la France, et sur les côtes opposées de l'Angleterre, dans le mois de mai, on roit de loin les belles fleurs rouges du sainfoin; le sous-sol de ces parag'es est composé de craie et c'est là que cette plaute réussit le mieux. Țoilà pourquoi ceux qui cultivent le sainfoin ici, où la craie n existe pas, doivent toujours le sampoudrer arec du plâtre, car la craie est un carbonate de chaux et le plâtre est un sulfate. La marne est ici très utile.

La graine de sainfoin se vend sous deux for mes : en balles, et nue ou égrénée. De cette dernière, 40 livres suffiront pour l'ensemencement d'un acre ; mais, en balles, il faut en semer $3 \frac{1}{2} \mathrm{mi}-$ nots. Il faut enterrer cette semence assez profondément.

En 1880, M. A. R. J. Fust sema une petite pièce de sainfoin. Malgré les araries que les gelées et les dégels lui firent subir, malgré sa destruction partielle, ce qui échappa profita merveilleusement au printemps, et le sainfoin fut prêt à être fauché dix jours plus tôt que le trèfle rougge du champ roisin. 
Pour en faire du foin, on doit faucher le sainfoin quand les fleurs sont à la veille de s'épanouir. Afin d'obtenir un plus fort rendement des prairies semées de sainfoin, il conrient de joindre ì cette légumineuse le fromental. Cette graminée se développe très vite et donne, dès la première année, un produit considérable; la deuxième année, le produit diminue sensiblement et les plantes commencent à disparaitre. La quantité de semence à ajouter au sainfoin est de 10 livres.

$\mathrm{Si}$, par hasard, au printemps de la première année, après l'ensemencement, le sainfoin n'a pas l'air bien vigoureux, il ne faut pas désespérer. Si le terrain est libre de mauvaises herbes, le sainfoin reprendra bravement arant la fin de la belle saison, et, la troisième année, il occupera toute l'étendue du champ.

Mais (blé-d'Inde).--On a tant discuté les qualités de cette espèce de fourrag'e, dans les conrentions de la société d'industrie laitière et dans les journaux d'agriculture, qu'il n'est pas nécessaire de nous étendre sur ce sujet. Mais on nous pardonnera si nous osons faire observer, conformément à l'opinion de M. Fust et de M. Tuck, fermier de MM. Dawes, de Lachine, Montréal, qui a toujours au moins cent cinquante bêtes à cornes à 
surveiller, qu'un arpent en trèfle rouge et dactyle mêlés, fauché en vert, fera produire autant de lait et de meilleure qualité, qu'un arpent et demi de blé-d'Inde vert. Nous ne parlons pas ici du bléd'Inde destiné au silo, où les épis, presque mûrs, sont hachés et mêlés arec les feuilles et les tiges, mais du maïs rert que l'on fauche pour donner aux raches dans le cours de l'été. Il ne faut pas oublier que le blé·d'Inde, arant l'époque du lustré, n'est qu'un fourrage rempli d'eau. "Ce n'est qu'un moyen côteux de faire boire ros raches que de leur donner du blé-d'Inde très rert," dit M. W. Robertson, de la Ferme Expérimentale. On peut faucher le trèfle et le dactyle trois fois dans le courant de la saison, et chaque coupe doit domner au moins six tomnes de fourrage rert, ou, en tout, dix-huit tonnes.

Nous renons de dire que le blé-d'Inde fauché trop rert ne contient guère de matières nourrissantes; il ne faut donc le couper que lorsqu'il est à peu près mûr, mais alors une partie de sa tige est derenue dure, coriace, fibreuse, indigeste et ne saurait être profitable. C'est poụrquoi il faut toujours donner la préférence aux fourrages verts mentionnés en premier lieu.

Mais comme on peut être tenté quelquefois de faire usage de blé-d'Inde, et que le blé-d'Inde 
est même presque indispensable pour le silo, il importe de comnaitre celui qui est le plus nutritif.

D'abord, on ne doit jamais employer que des plantes mûrissant parfaitement sous notre climat, si l'on veut en obtenir tout le profit qu'elles peuvent donner. Pour cette raison déjà, le blé-d'Inde canadien doit être celui dont nous devous nous servir. Ensuite, l'aualyse a prouré que notre bléd'Inde canadien, surtout le jaune, est beaucoup plus riche que celui provenant des contrées plus chaudes. Le blé-d'Inde canadien cultivé à la ferme expérimentale du Minnesota, doublait en matière nutritive le blé-d'Inde américain. Et le professeur Goegman a démontré que le blé-d'Inde de l'Est valait deux fois le blé-d'Inde de l'Ouest.

Le blé-d'Inde demande un terrain meuble, très fertile, riche surtout en acide phosphorique et en azote. On doit semer les grains à trois pieds de distance, ce qui fait à peu près un ảemi-minot à l'acre, si on veut avoir un fort rendement de bonne qualité.

FoURRAGES VERTS MOINS UTILES QUE LES PRÉCÉDENTS. - Ce sont:

L'avoine ;

Les féveroles;

Les vesces ;

Les lentilles;

Les pois. 
L'avoine, qu'elle soit donnée verte, ou sèche, à l'état de foin, le tige seule, ou arec le grain, ou le grain seul, diminue la sécrétion du lait. Il en est de même des pois.

Les féreroles, (en vert), de même que toutes les espèces de fères, ne doirent pas non plus être données aux raches laitières, d'abord parce que ces dernières ne les mangent qu'avec répugnance et qu'il est bien connu qu'il ne faut pas forcer la natu$r e$, les animaux connaissant ce qu'il leur faut bien mieux que nous; ensuite, "parce qu'il est admis géenéralement que les fèves ont une influence nuisible sur les qualités du beurre, qu'ils rendent sec et amer." (Extrait d'un ouvrage danois cité dans le rapport de M. G. A. Gigault).

Les resces ne valent pas beaucoup mieux; coupées en vert, elles ne doirent être données aux vaches laitières que hachées et bien mélangées avec de la bonne paille ou de la balle d'aroine bien propre, parce qu'elles contiennent, de même que les lentilles, un principe amer qui nuit aux vaches et à la cualité de leur lait. Domnées à l'état de foin, plus mûres, elles ont d'autres inconvenients, dont l'un est de faire maigrir sourent les raches. Il vaut donc mieux ne pas les employer.

La lentille constitue un meilleur fourrage 
que la resce, mais elle ne vaut pas les bons foarrages verts mentionnés dans cet article.

MaNière de servir LES FourRages verts AU BETAIL. - On ne derrait jamais distribuer les fourrages verts au bétail sans les aroir passés au hache-paille afin de les po:voir mélanger très bien avec du fourrage sec, bon foin, bonne paille, son, tourteau de coton, de lin. De cette manière non seulement il n'y aura rien de perdu, non seulement la quantité de lait sera beaucoup augmentée, mais surtout la richesse du lait en crème sera beaucoup plus forte, et la santé de tout le bétail sera florissante, robuste. Enfin, c'est un des principaux moyens d'obtenir un bétail qui surpassera tellement le bétail des autres pays, que tous les étrangers nous l'envieront. 


\section{IVII. LES BELLES PELOUSES}

\section{DFS PARCS ET DES GRANDS JARDINS}

\section{GAZONS D'AGREMENT}

Le plus bel attrait des jardins et des parcs, ce sont incontestablement les pelouses. Celles-ci sont aux jardins ce que les tapis sont aux salons; de là, selon M. Van Hulle, l'expression "un gazon comme un tapis." Seulement, pour que cette expression soit applicable, il faut que l'herbe rienne bien et surtout qu'elle soit entretenue d'une façon turt à fait irréprochable. Dans notre pays, cela se rencontre rarement, tandis qu'en Angleterre les belles pelouses sont de rigueur, se roient presque partout.

Tout amateur ayant visité ce pays a voulu, en rentrant chez lui, imiter les pelouses anglaises; rarement il a réussi complètement, surtout arec si peu de peines; car, en somme, nous sarons que, làbas, on est loin d'y mettre autant de soins qu'on serait tenté rle le croire. On rase constamment, il est rrai, on roule, on tasse, on durcit les contours, mais c'est à peu près tout. Iih bien! on le sait par 
expérience, cela est loin de suffire chez nous. Ṃême en arrosant nos pelouses de temps à autre nous ne parvenons pas à les faire ressembler tant soit peu aux gazons anglais (english lawns.)

Que de fois nous nous sommes demandé quelle pourait bien en être la cause. Sans aucun doute, le climat, qui est brumeux, et la nature du sol, qui est humide et calcaire, sont pour la plus grande part dans la réussite générale des gazons, chez nos voisins d'outre-mer. Sans doute, encore, la façon dont ils préparent leur sol, le choix de leurs herbes, l'entretien de leurs pelouses, tout cela se fait arec soin. Mais, encore une fois, quantité d'amateurs ont imité ces procédés sans succès complet, et surtout durable. M. Van Hulle, dans son jardin qui n'est pas très étendu, (et l'on comprend que, sur une aussi petite échelle, il est relativement facile d'apporter tous les soins possibles,) est parrenu à aroir ce qu'on peut appeler un beau gazon. Cependant, à son vif regret, il n'a pu arriver à un résultat tout à fait satisfaisant. Il est vrai que son jardin est assez éleré, fort en pente, et que le sol en est léger; mais pour une si petite étendue, ces défauts ne doivent pas être des obstacles insurmontables.

Où faudrait-il donc chercher les causes de ces 
demi-résultats? Dans le climat d'abord, dans le sol ensuite, et enfin dans la manière d'opérer.

Presque toutes les herbes aiment la fraîcheur, non seulement aux racines, mais aussi aux feuilles; il s'en suit que notre situation, ne répondant pas à ces conditions, péche par la base. Voilà la preunière et la plus grande difficulté, contre laquelle on lut. tera souvent en vain.

Pour ce qui est du sol, plus il sera sablomneux, plus aussi il sera défarorable; plus, au contraire, il sera argilenx et mieux les herbes y réussirnnt, s'y étaleront surtout; ce qui est l'essentiel.

Si donc, sur une côte aride et sablonneuse, il est presque impossible d'obtenir un beau gazon, une rallée basse et argileuse y est très propice. Cela ne suffit pas cependant : il faut encore bien préparer le sol et soigner les herbes.

\section{Le tassement}

C'hacun peut observer qu'ordinairement c'est lo long des sentiers, où la terre est plus dure, qu'on trouve le plus beau gazon; ne pourrait-on pas en conclure quele sol n'a nullement besoin d'être İabouré pour créer les pelouses? Non, ce serait une erreur; il faut non seulement un labour, mais il en faunt plusieurs, et même assez profonds. Seule- 
ment il importe de laisser le sol longtemps s'asseoir, se tasser; puis avant de le semer, il convient de le piétiner, de le rouler, de durcir les contours assez fortement pour que le talon de la botte n'y pénètre plus. Ensuite on continue la même opération à mesure que le jeune gazon lève et se forme. Q $u$ 'on ne se fasse pas l'illusion de croire pouvoir réussir dans une terre légère et sans consistance; non, il n'y aura de succès durable qu'autant que le sol aura été suffisamment durci et demeurera tel.

Les contours des pelouses à créer ne pourront être durcis que s'ils sont en talus, même dans les terrains compacts. Ailleurs, il n'y faut pas songer. Que faire alors? On peut employer plusieurs moyens, comme de faire des bordures avec des tranches de gazon pris le long de la voie publique, en ayant soin de bien réunir les sondures, de les arrêter par des chevilles. et de les protéger contre la sécheresse, contre les rayons solaires, tant que le tout n'est pas pris en une seule masse. Ça demande beaucoup d'ouvrage et de soins, mais c'est bien beau.

N. B. Tout ce que nous arons dit touchant la création des gazons, moins ce qui regarde les contours, s'applique également aux prairies; cela va de soi. 


\section{Semis}

Quelques personnes ne sêment que du raygrass pur, qui forme, à la vérité, de jolis gazons; mais pour la première année seulement, cette herbe ne résistant pas au climat de notre Prorince. Il est dous préférable de semer un mélange spécial, si l'on reut obtenir une pelouse persistante.

Ce mélange étant un composé de graminées et de légumineuse. forme un gazon beaucoup plus fin et plus serré que le ray-grass pur, ainsi que toute autre herbe, graminée ou lég umineuse, semée seule, séparément; il résiste mieux à la sécheresse; il dure également plus longtemps.

M. James Fletcher, botaniste des Fermes Expérimentales, dans dans son liapport jour 1892, s'exprime ainsi :

"Nous sommes arrivés à la conclusion très satisfaisante que, pour créer de bons gazons en Canada, aucune graminée n'est à comparer arec le commun paturin des prés, qui garnit le bord des routes. Pour sa couleur vive, permanente, pour l'uniformité de la pousse et le moelleux de la texture, aussi bien que pour sa rusticité à toute épreure et sa résistance aux mauvais traitements, il n'a pas son pareil. Il a aussi presque intariablement été désigné comme le plus beau par tous 
ceux à qui on demandait leur opinion, soit qu'ils comnussent les difiérentes graminées, ou non. On s'en procure facilement la graine, qui est peu coùtense et presque toujours pure; car elle mûrit avant celle de la plupart des herbes qui croissent. parmi les graminées. A la fin de juin, quiconque reut s'en donner la peine peut, en une heure de temps, recueillir sur le bord d'un chemin assez de graines pour une grande pelouse. A u bout d'une semaine, eette graine sera assez sèche pour se détacher toute de la balle par le frottenent entre les doights, et on peut aussitôt la semer. l'our réussir à eréer un gazoh, il faut que le sol ait une bome profondeur et soit bien drainé, 11 faut labourer la surface, niveler, semer et passer le rouleau en automne. Au printemps, il faut de nouveau rouler.

"Il faut semer dru, afin que les feuilles poussent épaisses et fines, à raison d'au moins 3 ì $3 \frac{1}{3}$ boisseaux (minots) de graines de paturin des prés à l'acere, (la graine du paturin des prés pèse $\mathbf{1 4}$ livres au minot); on peut y ajouter $\frac{1}{4}$ de livre de trèfle blanc. Ni le sol est humide, on peut remplacer moitié environ de la quantité ci-dessus par la graine de l'une des nombreuses agrostides ì ferilles fines, telles qu'A grostis stolonifera; mais la coulenr de cette dernière est un rert de teinte 
distinctement différente, et il faut se rappeler que l'agrostide, si elle convient mieux au sol, finira par supplanter le paturin."

Dans les très grandes pelouses, pour enterrer la graine on peut employer une herse légère; on recourre le semis d'un peu de terreau, si l'on en a à sa disposition, et on roule ensuite.

Le semis d'automne est préférable au semis de printemps.

\section{Le sarclage}

Bien que toutes les conditions ci-dessus aient été scrupuleusement remplies, et quelle que soit l'espèce de graines d'herbe qu'on ait semée, on verra peu à peu apparaitre dans la pelouse, non seulement une foule de plantes qui ne sont pas des herbes et qu'il faut, par conséquent, arracher au plus tôt, mais aussi des herbes dont on ne peut tolérer la présence à aucun prix. De ce nombre sont le chiendent, et toutes les plantes indistinctement qui semblent ronloir dominer par leur régétation, ou être peu disposées à s'étaler, à faire gazon, comme on dit. Sauf à tasser de noureau, il faut donc, au besoin, arracher hardiment tout ce qui ne convient pas, sans s'inquiéter des petits vides que l'on fait ainsi; ils se rempliront par la suite. $\mathrm{Si}$, cependant, ces vides sont trop considérạ- 
bles, il y faudra répandre quelques graines ou, ce qui vaut mieux, y planter un petit morcean de beau gazon, qu'on trouve presque toujours, sans peine, le long de la voie publique.

C'est déjà un grand point d'être parvenu à avoir une herbe, encore clair-semée peut-être, mais plus or moins régulière et mblant se plaire quelque peu dans le terrain. L'espèce d'herbe doit convenir au sol: cela est tellement important, qu'en Angleterre, des amateurs façonment simplement leur terrain, puis l'abandonnent à lui-même. Comme toujours, ce terrain ne tarde pas à se couvrir de régétation. Alors, ils arrachent au fur et a mesure toutes les herbes qui ne conviennent pas. C'est ainsi que, dit-on, même dans les situations les plus défavorables, on débute pour former les gazons les plus beaux et les plus durables.

Et de fait, nous n'en sommes pas absolument surpris. En effet, tous les jours n'avons-rous pas l'occasion de voir du beau gazou le long de la route, où aucun soin quelconque ne lui est donné tandis que, dans nos jardins, malgré toutes nos peines, nous n'arrirons qu'à un demi-strccès? On doit en chercher l'explication moins dans la composition de notre sol, qui ne conviendrait pas à notre herbe, que dans la nature de notre herbe, 
qui ne convient pas à notre sol. (Voir le troisième paragraphe, au mot semis.)

\section{La tonte}

Dans le principe, on ne doit pas songer à laisser monter l'herbe pour l'utiliser, du moins comme fourrage fané : il faut, au contraire, durant tout l'été, la raser très sourent arec une de ces ingénieuses machines tondeuses ou faucheuses pour gazon, qui sont aujourd'hui d'un usage général dans les jardins et les parcs bien teinus. On pourrait aussi enlever, partout où ils existent encore, ces écritaux si peu rationels portant: "Défeuse de circuler sur les pelouses". Qu'on empêche de faire des sentiers à travers les gazons, ce que le public ne fera d'ailleurs pas quand l'artiste aura été assez bien inspiré pour tracer couvenablement les allées,-soit; mais, à part cela, plus un gazon est piétiné, mieux il se portera, à moins qu'on ne s'attache à l'user littéralement. Si, en outre, il pleut assez sourent, ou si, à défaut de pluie, on arrose suffisamment, le soir tard, surtout arec un peu d'engrais liquide, on peut être certain d'une réussite complète.

Les composts, les balayures, les boues et les limons

Toici, d'après II. Tan Hulle, ce qu'il y a de mieux à faire, indépendamment de ce qui a été 
recommandé ci-dessus, non seulement pour créer une belle pelouse dans les situations farorables, mais encore pour lui donner de la durée, même dans de très mantaises conditions:

"Ne pourrait-on pas attribuer, en très grande partie, la beauté du gazon le long de la roie publique à la poussière que le rent $\mathrm{y}$ chasse et qui recouvre et abrite constamment les racines supérieures, les plus importantes, au fur et à mesure, pour ainsi dire, qu'elles se forment, tandis que, dans nos pelouses et nos jardins, ces racines supérieures sont rôties par le soleil? Dans l'affirmative,-et nous croyons que cela n'est pas douteux,-il suffirait d'imiter plus ou moins ce qui se passe dans la nature, c'est-à-dire de répandre de temps à autre une légère couche de terre sur le gazon. Il va de soi que, plus cette terre sera fertile ou substantielle, mieux cela raudra; toutefois, au besoin, et durant l'été surtout, toute terre, quelque légère qu'elle soit, doit produire un excellent effet. Tâchous done d'aroir toujours quelque part en dépôt un immense tas de terre et chaque-fois que, par ci par là, une place laisse à désirer, répandons-y environ quatre lignes (un demi pouce), de cette terre, durant tout l'été.

" La grande saison, cependant, pour appliquer aux pelouses ce soin important, c'est l'hiver. 
Charriez done sur rotre gazon, durant les mois de décembre, janvier ou fétrier, soit du fumier quelcomque, soit des balayures de rues, soit ce qui surpasse tout, des boues de dragages ou limons des cours d'eau.* Quand ces boues sont bien grasses et qu'on contiuue à en mettre tous les hirers à l'épaisseur d'un demi-pouce, on finira par aroir un bean et durable gazon dans les terrains les moins propices; nous sommes même d'aris qu'il n'y a que ce moyen pour réusir. C'est que la plupart des autres ingrédients organiques auxquels on recourt d'habitude, se décomposent et, par conséquent, s'en ront totalement, tandis que le limon, tout en engraissant, consolide en même temps le sol par Ins substances minérales dont il est composé et qui restent acquises.

"Il importe done d'amasser du limon, durant l'été, par exemple. Laissez ces boues s'essuinter (se ressuyer) d'abord; remuez les deux ou trois fois à un mois d'interralle et, l'hirer renu, conduisez et répandez-les sur les pelouses sans rous inquiéter beaucoup si elles restent en grosses croûtes. Ters le commencement d'avril, passez sur rotre gazou avec la herse ou le rateau, et la boue d'autrefois s'émiettera d'elle-même et constituera à la surface du gazon une légère couche de terre grasse, à travers laquelle l'herbe ne tardera pas à pousser, à

(*) Quanrl aux boues et limous des fossés d'égouttement, qui eintiennent toujours heaucoun de graines de mauraises berbes, on ne peut les employer qu'aprè en aroir fait un compost iaree de la chaux, cendres, ete.,)--suffi-amment muri pour qu'il n' $y$ ait plus de graines rirantes. 
derenir vert foncé et à être de plus en plus serrée. Roulez, tassez, consolidez alors et le gazon et les contours de rechef, entretenez bien la tonte, et, peu à peu, vous formerez un gazon indestructible. Il ra de soi que, dans les amnées très sèches, il devient nécessaire, à moins de voir souffrir plus ou moins la pelouse, d'arroser le soir. Il est rrai encore qu'administrer un peu d'engrais liquide par un temps pluvieux ne peut qu'être farorable; mais le plus grand point, c'est de limoner, si nous pourons employer ce terme, tous les ans quelque peu."

Nous renons de dire que le temps le plus propice pour transporter ces boues sur les pelouses, est l'hiver. En roici les raisons; la première, c'est que par là on érite de hriser le sol des pelouses arec les roues des roitures d'été, qui fouleraient trop riolemment et trop inégalement le terrain; ensuite, et c'est la principale raison, parce que l'hirer, sous notre climat rigoureux, est le meilleur scarificateur (pulvérisateur), qui, le printemps arrivé, a achevé soin travail et laisse, en se retirant, un engrais admirablement préparé pour être répandu sur les pelouses. De fait, c'est le printemps, de boune heure, arant la reprise de la régétation, qu'il couvient de limoner les pelouses. 





\section{TABLE DES MATLERES}

A.

Ausérine...................................... 13

Assainissement................................. 89

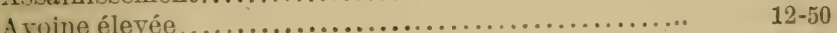

Aroine jaunâtre... .......................... 19-21-51

Avoine en vert ............................... 115

B.

Balayures.......................................

Boucage à grandes feuilles......................

Bules

Brome doux ................................ 20,2t

Brome inerme .............................. $52-163$

Brunelle .................................... 13

C.

Carotte sauvage

Chaux ............................... 31-98-112-188

Choix des plantes............................ 41

Chrysanthème des moissons......................

Centaurée des prés.............................. 13

Composts.................................... 104

Conditions climatériques des cultures fourragères... 10

Crenirie .

Crête de cor

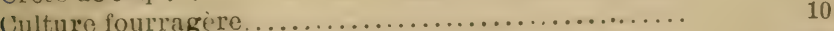

Cultures preparatoires........................... 91

Cumin des prés ... ......................... 13-20 
D.

Dactyle................... I9-23-29-39-40-43-45-52-138-163-169 Déloncement............................... 102-103-111

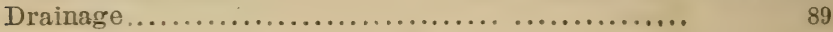

E.

Engazonnement naturel........................ 11

Engazonnement naturel perfectionné.............. 18

Engazonnement artificiel....................... . 27

Engrais................................... $\quad 95-100$

Engraissement du sol.......................... 36

Entretien des prairies........................... ${ }_{12}$

Eperriaires.................................. , 20

Epis......................................... 44

Exposition du champ............................ 36

F.

Fauchage des prairies $\quad$...................... 151-153

Fenaison................................. ${ }_{15} 153157$

Fétuques................................... 45

Fétuque des prés........................... 19-24-29-13s

Fétuque ovine ............................... 19-23-29-51

Fétuque durette.......................... 53

Fétuque élevée................................. 51

Féveroles .................................. 175

Fléole des prés........................ 29-31-36-39-40-44-46

Flouve odorante............................ 11-13-19

Foin.................................... 144

Fourrages verts ........................... 161-163

Fromental......................... 12-13-29-39-40-13-45-50

Fumure................................. 96

G.

Gaine .....................................

Galéope des champs.......................... 13

Gazons d'ornement.............................. $\quad 178$

Gesse des prés................................ 13 
Glumes.................................. 14

Glumelles................................... 4

Graminées ............................ 29-31-39-41-42-14

Graminées étrangères...................... 3t-46-50

Graminées indigènes.......................... 31.46

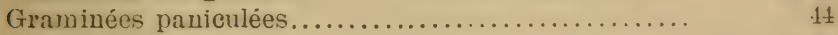

Graude marguerite........................... 24

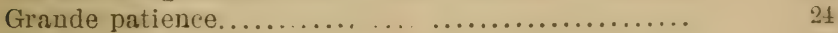

Graude pimprenelle.......................... 20

$\mathrm{H}$.

Herbe ................................. 144

Hersage des prairies......................... 124

Houlnue lain`use...................... 11-13-19-20-21

L.

Légumineuses........................... 29-31-34-57

Légumineuses (petites]...................... 43

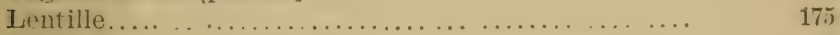

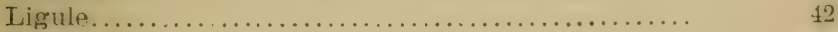

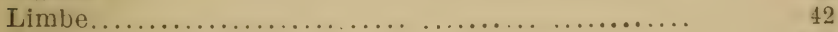

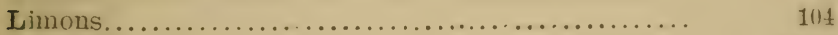

Lupuline ................................. 13-23

Luzerne ............................ 27-28-31-163-166

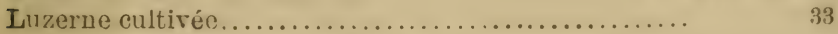

II.

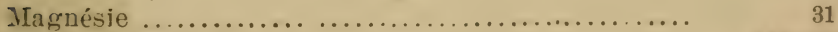

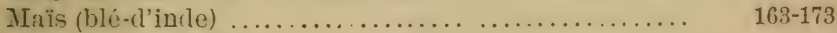

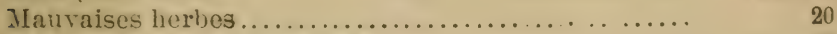

Mélanges, thérrio et caleul des................... 27

MIélauges, quantité relative de graines dans les...... $33^{\prime}$

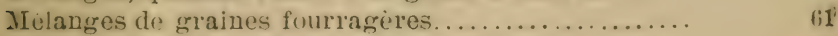

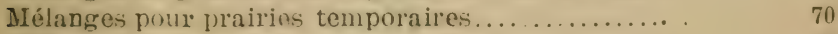

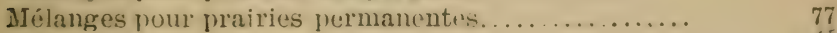

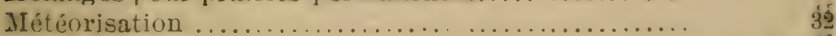

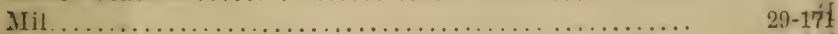

Mousses, destruction des...................... 1sug

Myosutis....................................... 13- 
o.

Oseille des prẻs

Oraire.

Papilles.... .................................... 14

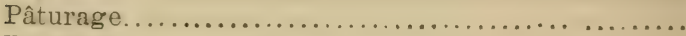

Paturins $13-19-45$

Paturin comprimé.

Paturin tardif.

Paturin commun. $11-17$

Paturin des prés

Pédicello

Pelouses

Persil d'âne...

Petite oseille

Pissenlit.........................................

Plâtre.

Plantain lancéolé

Pois

Potasse.

Poudre d'os....

Q.

Quantité à semer.

Quantité de semence à l'arre (en lirres et onces)..... .

R.

Ray-grass anglais

Ray-grass d'Italie...........................

Renoncule rampante.......................... 
Renoncule âcre

13-20-34

lienouée persicaire.

Restitution au sol.

106-110

Roulage des prairies.

122

S.

Sainfoin

27-29-31 . 61-163-171

Sainfoin cultive

Scribe des bois.

Seigle

Semaille tardive

Semailles, époque des............................

Semences, achat des

Semence à l'arpent.

Semence vieille, légère, etc ......................

Semence saine, fraiche, pleine et pesante.............

" en terre forte.............

" en terre légère.

Semis de graine de foin.........................

Semoir.... .................................

Sétaire glauque ..............................

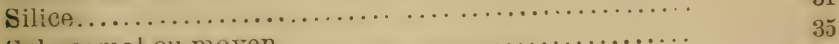

Sol normal ou moyen.. .. . . . . . . . . . . . . . . . . .

Sous-sol...................................

Stigmates..................................... $\quad 129$

Sulfate de fer..............................

Supplément.

$\mathrm{T}$.

Théorie et caleul des mélanges.

Trèfle alsique (bybride). .

Trèflles et graminées.

Trèflle blane

Trèfle jaune.

T'rèfle ruuge

Vesces. 





